\title{
Long-Term Temporal Dynamics of Synaptic Vesicles
}

\author{
Dissertation \\ for the conferral of the degree \\ Doctor rerum naturalium
}

by the Georg-August-Universität Göttingen

within the doctoral programme IMPRS for Molecular Biology

of the Göttingen Graduate School for Neurosciences, Biophysics, and Molecular Biosciences

submitted by

Sven Truckenbrodt

from Coburg, Germany

Göttingen, September 2016 



\section{Examination Board}

\section{Thesis Committee}

Prof. Dr. Silvio O. Rizzoli

Department of Neuro- and Sensory Physiology

University of Göttingen Medical Centre, Göttingen, Germany

Prof. Dr. Reinhard Jahn

Department of Neurobiology

Max Planck Institute for Biophysical Chemistry, Göttingen, Germany

Prof. Dr. Blanche Schwappach

Department of Molecular Biology

University of Göttingen Medical Centre, Göttingen, Germany

\section{Extended Examination Board}

Prof. Dr. Nils Brose

Department of Molecular Neurobiology

Max Planck Institute for Experimental Medicine, Göttingen, Germany

Prof. Dr. Peter Rehling

Department of Cellular Biochemistry

University of Göttingen Medical Centre, Göttingen, Germany

Dr. Camin Dean

Group of Trans-synaptic Signalling

European Neuroscience Institute, Göttingen, Germany 

The good life is one inspired by love and guided by knowledge.

Bertrand Russel 



\section{Affidavit}

I hereby declare that I prepared this dissertation independently, and with no other sources and aids than quoted.

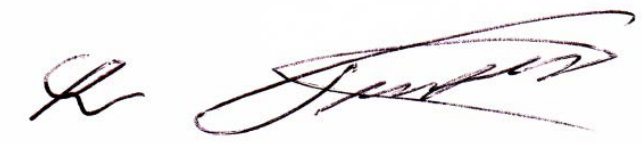

Sven Truckenbrodt 

Dedication

Meinen Eltern. 



\section{Table of Contents}

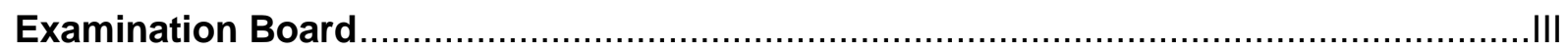

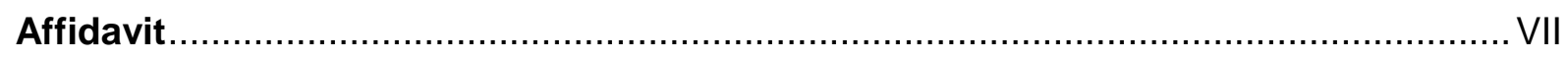

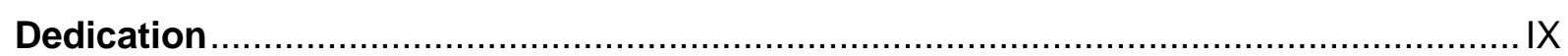

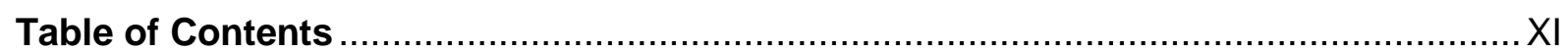

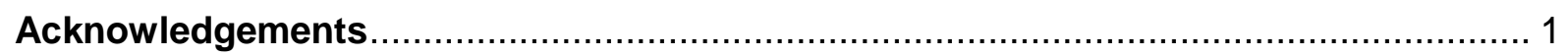

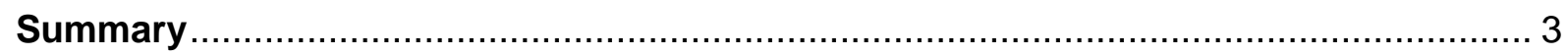

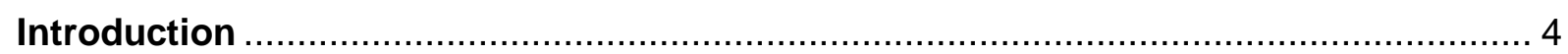

1.1 Scope of this Thesis: the pool conundrum ................................................. 4

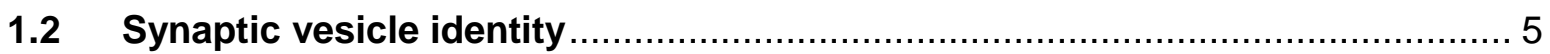

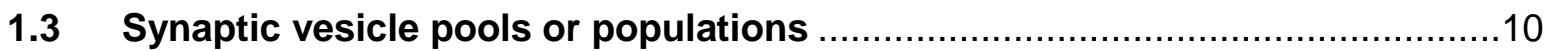

1.4 Population identity and functional transitions .............................................

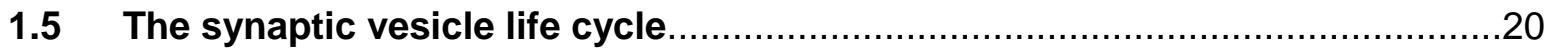

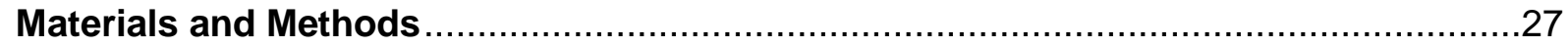

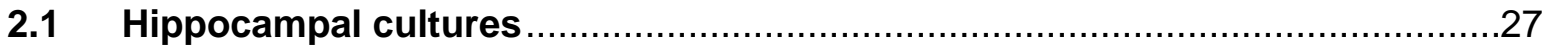

2.2 Live-cell tagging of synaptic vesicles with antibodies ...............................28

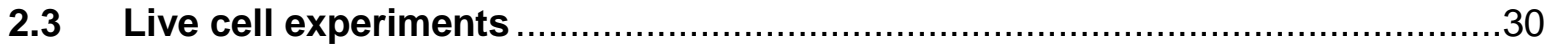

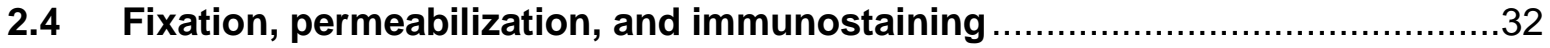

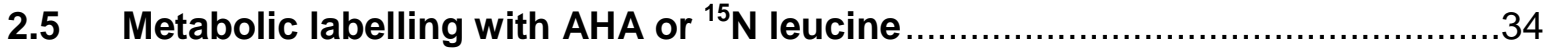

2.6 SNAP-tag labelling and TEV protease cleavage in living neurons ..................35

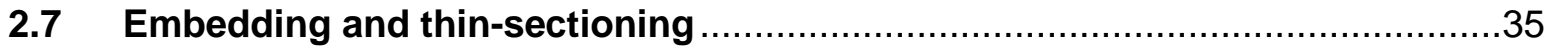

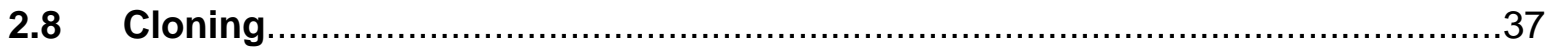

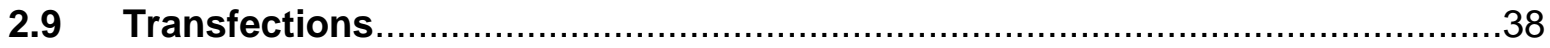

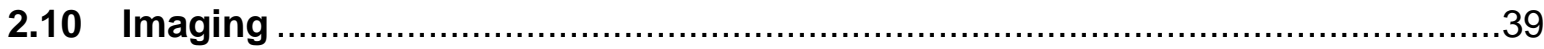

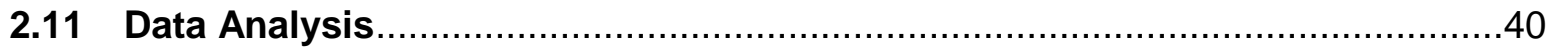

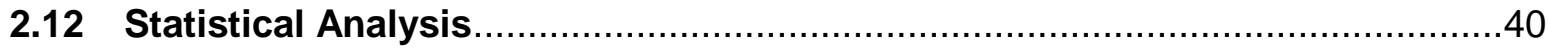

2.13 Calculation of SNAP25 copy numbers on young and old synaptic vesicles ...40

2.14 Prediction of protein damage from protein lifetimes ..................................41

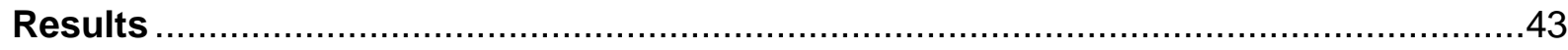

3.1 Live-cell tagging with antibodies allows investigation of the synaptic vesicle

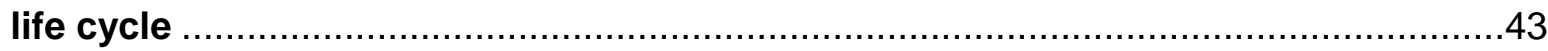

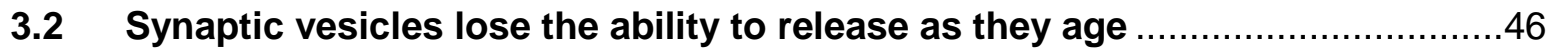

3.3 Releasable synaptic vesicles are metabolically young and the synapse is

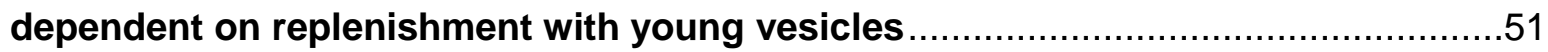

3.4 Synaptic vesicles release $\sim 260$ times before becoming inactive ...................56

3.5 Ageing synaptic vesicles get contaminated with SNAP25 from the cell membrane 
3.6 CSPa is a quantitative bottleneck for synaptic vesicle release and is blocked by vesicular SNAP25 in a molecular timer mechanism.

3.7 Synaptic vesicle inactivation through a timer for use prevents the use of

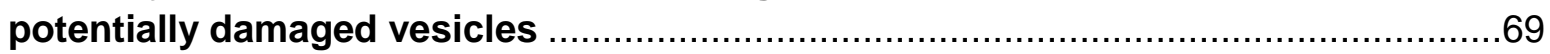

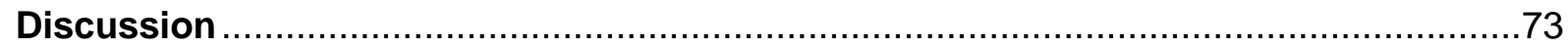

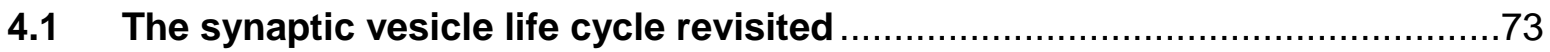

4.2 Timing and quantification of the synaptic vesicle life cycle .........................77

4.3 A timer of organelle usage: SNAP25 contamination and the quantitative CSPa

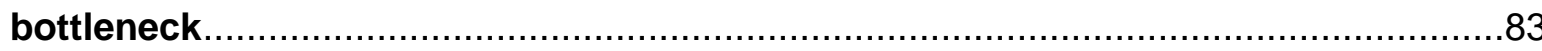

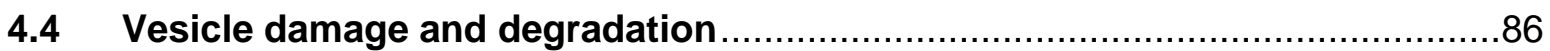

4.5 Predicting protein damage and pre-emptive inactivation of organelles: a novel concept for cell biology .............................................................................. 90

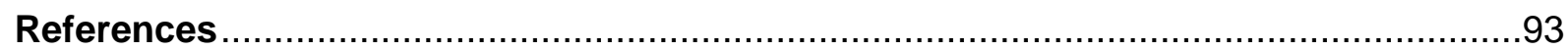

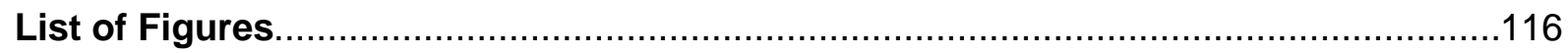

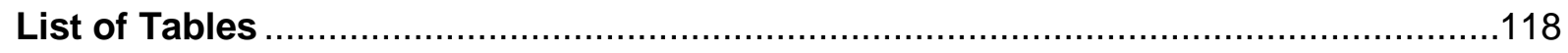

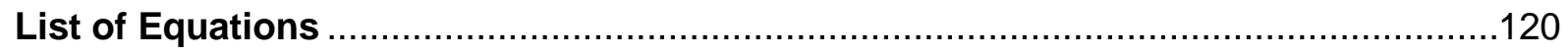

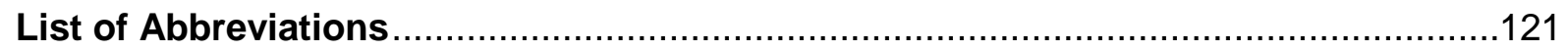

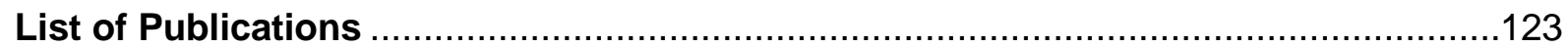

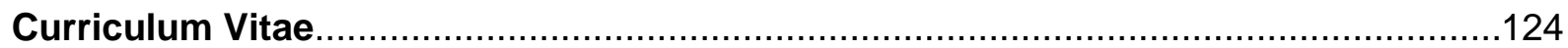





\section{Acknowledgements}

I am greatly indebted to Silvio Rizzoli for his outstanding and exceptional supervision during my PhD project. His advice on the scientific aspects of my project was invaluable, while leaving me enough room to develop and pursue my own ideas. He was always available for experimental help, theoretical discussions, and questions on data analysis at the oddest hours and in disregard of any of his own workload. I further thank him for providing most MATLAB scripts I used for data analysis during my PhD project. I also thank him for his wit and his humour, and for wide-ranging discussions beyond science. But most importantly, I thank him for providing me with countless opportunities to speak at conferences, to form a scientific network, to understand the world of academia, and to develop as a scientist. His efforts in this regard went beyond anything a PhD student could reasonably expect. I could not have wished for a better supervisor.

I thank my Thesis Committee members, Reinhard Jahn and Blanche Schwappach, for their input during Thesis Committee Meetings. I further thank Reinhard Jahn for mentoring advice before and during my $\mathrm{PhD}$ regarding several key decisions, and I thank Blanche Schwappach for experimental collaborations.

I am indebted to my Biology teacher, Christian Wegmann, and my German teacher, Klaus Mischke, for providing the basis for my higher education and motivating me to go to university. I further thank Erich Buchner and Alexander Kapustjansky, the supervisors of my Bachelor's Thesis, for guiding my first steps into research.

I am indebted to Steffen Burkhard from the IMPRS Molecular Biology office for his tireless work in organizing the greatest graduate school I can imagine. He breathed life into our programme and I do not want to imagine it without him. I am indebted to him and to the entire programme for providing us students with a perfect study environment that was both scientifically stimulating and socially warm. I am further indebted to the IMPRS Molecular Biology for providing opportunities such as organizing our own conference, Horizons in Molecular Biology, visiting our partner programme at the Weizmann Institute of Science in Israel, and organizing culture nights, excursions and other social events. I thank the entire GGNB for organizing valuable methods courses and soft skills courses. I further thank the GGNB for providing me with Travel Grants and an Excellence Stipend.

I cannot speak about the IMPRS Molecular Biology programme without mentioning my fellow students, both from my own batch and the batches before and after us. You made my years 
in Göttingen, especially our first year of coursework, one of the most memorable experiences of my life. In particular, I would like to thank Lena, Muna, Kanika, and Zhenya from my Method's Course group for three very enjoyable and fun months. I will always treasure our wide-ranging womanly discussions.

I am indebted to Eugenio Fornasiero for countless fruitful hours of discussion of my project and invaluable advice on my project and on the world of academia. I thank Christina Schäfer for reliably providing excellent primary rat hippocampal neuron cultures every week. I thank Abhiyan Viplav for providing the data on changes on synaptic vesicle protein levels over time during his work for his Master's Thesis in our lab. I thank Sebastian Jähne and Angela Vogts for helping to organize and perform the nanoSIMS experiments on the age of synaptic vesicles that are part of my PhD project. I thank Annette Denker for introducing me to my PhD project and for supervising my lab rotation. I thank Bennjamin Wilhlem for introducing me to his project on the organization of the synapse, which became indispensable for my Thesis project. I thank Natalia Revelo for providing me with an introduction to mCLING and with the opportunity to contribute to her project. I thank Sinem Saka for introducing me to metabolic labelling of proteins with AHA.

My PhD years would not have been the same without my amazing fellow lab members. I thank Manuel for many ludicrous scenes and general absurdity; also for his garden parties. I thank Martin for introducing me to D\&D. I thank everyone from the lab, past and present, for many enjoyable discussions, lab trips, games, barbeques, and cocktail nights.

I left my most heavy debt for the end. Ina, you have been with me through the best times and the worst times and made it all worthwhile. I can only hope that my presence in your life has meant as much as yours in mine, because I certainly cannot hope to repay this debt in any other way. 


\section{Summary}

Neurotransmission requires the release of neurotransmitters from synaptic vesicles. This occurs via fusion of the vesicle to the pre-synaptic membrane upon stimulation. However, not all synaptic vesicles are equally releasable, and it has long been debated why the majority of synaptic vesicles do not respond to physiological levels of stimulation. I demonstrate here, using live-cell antibody-tagging in rat hippocampal cultures, that only young synaptic vesicles are releasing neurotransmitter, and that they become more reluctant to release as they age. This inactivation of synaptic vesicles is not strictly an ageing-dependent process, but conditional upon vesicle usage. I report here that synaptic vesicles release $\sim 260$ times, on average, before becoming inactive, and that increasing usage frequency speeds up inactivation. The inactivation is caused by contamination of synaptic vesicles with the cell membrane protein SNAP25. SNAP25 can interact with the vesicle protein CSPa in ciscomplexes on the vesicle itself. This sequesters CSP $\alpha$ and prevents the formation of transcomplexes with SNAP25 on the cell membrane. This trans-interaction, however, promotes vesicle fusion to the cell membrane. The more often a vesicle has fused to the cell membrane, the higher its chance is to be contaminated with SNAP25, and the less competent it is for future rounds of release. The inactivation of ageing synaptic vesicles is presumably coupled to usage to remove potentially damaged synaptic vesicles from neurotransmission. This hypothesis is strengthened by the observation of endocytosis defects and neurite degeneration when aged vesicles are forced to release. I further provide several timing parameters for key events in the life of synaptic vesicles, which can serve as a framework towards a quantitative model of the synaptic vesicle life cycle. 


\section{Introduction}

\subsection{Scope of this Thesis: the pool conundrum}

Neurotransmission is the central process of synapses. To achieve the transduction of signals from neuron to neuron, neurotransmitters are released at the sending pre-synaptic side and detected at the receiving post-synaptic side. Neurotransmitters are stored in and released from a specialised organelle: the synaptic vesicle. It consists of a lipid membrane, interlaced with a variety of proteins for the exchange of ions and neurotransmitters between the vesicle lumen and the cytosol, fusion of the vesicle to the cell membrane, retrieval of the vesicle by endocytosis, interaction with the cytoskeleton, and regulatory processes. Most synapses contain at least hundreds, and sometimes hundreds of thousands, of these synaptic vesicles (Truckenbrodt and Rizzoli, 2015). They are arguably the best characterised and most completely understood organelle of any cell type: their function had been partially predicted even before their discovery (Birks and Maclntosh, 1961; del Castillo and Katz, 1954; Elmqvist and Quastel, 1965; Fatt, and Katz, 1952; Fatt and Katz, 1950), they were among the first organelles described structurally and functionally with electron microscopy (Ceccarelli et al., 1973; Heuser and Reese, 1973; Palade and Palay, 1954; De Robertis and Bennett, 1955) and super-resolution light microscopy (Westphal et al., 2008; Willig et al., 2006), their quantitative protein and lipid composition is known (Takamori et al., 2006; Wilhelm et al., 2014), and the function and structure of all their proteins has been solved down to atomic resolution in all but the most minute details (Jahn and Fasshauer, 2012; Südhof, 1995, 2004).

However, the behaviour of individual synaptic vesicles in the local population at the synapse has remained largely enigmatic. Early on during the investigation of neurotransmission, it was observed that only a fraction of all neurotransmitter is releasable (Birks and MacIntosh, 1961; Elmqvist and Quastel, 1965). After the discovery of synaptic vesicles, it was confirmed that some of them are not releasable, sometimes even under the most severe stimulation (reviewed in Denker and Rizzoli, 2010; Rizzoli and Betz, 2005). Based on these observations, synaptic vesicles have been classified into different functional pools, according to their release behaviour: vesicles of the "recycling pool" are releasable and can undergo multiple rounds of release, each followed by recycling of the vesicle components, while vesicles of the "reserve pool" are inactive and can only partially be made to release under severe supra-physiological stimulation (Alabi and Tsien, 2012; Denker and Rizzoli, 2010; Rizzoli and Betz, 2005). Under physiological conditions in situ, only a minority of $<5 \%$ of all vesicles is releasable (Denker et al., 2011a; Körber et al., 2012). This has been extremely puzzling, because there is no readily apparent distinction between the releasable vesicles 
and the inactive vesicles. Releasable vesicles do not occupy privileged positions within the synapse (Rizzoli and Betz, 2004; Xu-Friedman et al., 2001), data on molecular differences is scarce and has never been sufficient to explain the functional distinction on a molecular level (Bal et al., 2013; Hua et al., 2011b; Ramirez et al., 2012), and the ultrastructure is no indication either (Harlow et al., 2013).

There is thus a significant gap in our understanding of the synaptic vesicle: functionally distinct synaptic vesicles are molecularly indistinguishable. To fill this gap, I set out to investigate the molecular differences that are responsible for determining the functional state of a vesicle. In other words: what makes a vesicle releasable, and what inactivates it? Starting from the observation that the same synaptic vesicles seem to be able to transition from the releasable state to the inactive state over time (Kamin et al., 2010; Richards et al., 2000, 2003; Rizzoli and Betz, 2004), I strived to elucidate functional and molecular changes throughout the entire life cycle of the synaptic vesicle, starting with its arrival at the synapse, and ending with its degradation. I determined that synaptic vesicles irreversibly switch from the releasable state to the inactive state, that only metabolically young vesicles are releasable, and that they become inactive after $\sim 260$ rounds of release. I further describe here how this switch is mediated by a molecular timing mechanism based on the contamination of vesicles with SNAP25 from the cell membrane and a quantitative bottleneck of the vesicular release co-factor CSPa.

\subsection{Synaptic vesicle identity}

It has been a matter of much debate whether synaptic vesicles can be regarded as stable units that can be considered to go through a life cycle, or whether their identity is lost with each release event due to intermixing with membrane resident vesicle proteins (up to $\sim 20 \%$ of vesicle proteins reside on the cell membrane at any time; Balaji and Ryan, 2007; Fernández-Alfonso et al., 2006; Granseth et al., 2006; Opazo et al., 2010; Revelo et al., 2014; Sankaranarayanan and Ryan, 2000; Wienisch and Klingauf, 2006). In other words: do synaptic vesicles remain on the cell membrane as stable patches of proteins and lipids after release and are recycled together, or do proteins and lipids disperse in the cell membrane upon fusion and do recycled vesicles re-assemble from a mixed pool of lipids and proteins from many previously released vesicles? I term the first model the "cohesion hypothesis" here, and the second model the "dispersal hypothesis".

Dispersal hypothesis. The main arguments for the dispersal hypothesis come from experiments in which fluorescently tagged constructs of synaptic vesicle proteins are overexpressed and observed in bulk at epifluorescence or confocal resolution during severe 
supra-physiological stimulation. The most common approach is to use a pH-sensitive protein, such as pHluorin (Miesenböck et al., 1998), trigger release (sometimes after quenching the surface fluorescence to achieve a better signal-to-noise ratio), and observe the dynamics of the fused pHluorin molecules on the surface. Most studies using this approach, on different synaptic vesicle proteins, found a rapid lateral movement of pHluorin-tagged constructs away from the site of fusion (Granseth et al., 2006; Li and Murthy, 2001; Sankaranarayanan and Ryan, 2000; Wienisch and Klingauf, 2006). The authors of these studies interpreted this as a disintegration of the synaptic vesicle upon fusion. Their main argument for this interpretation is that rapid movement of a clustered synaptic vesicle protein patches is unlikely. However, these studies did not address why the movement of a vesicle patch on the membrane should necessarily be slower than what was observed in their experiments. While diffusion is indeed slowed for proteins in clusters, it is still fast enough to account for the movement rates observed in these studies (Saka et al., 2014a). Another argument in favour of the dispersal hypothesis is the observation that a mixed population of formerly surface-resident and recently released vesicle material is retrieved following strong stimulation (Wienisch and Klingauf, 2006). However, since this was only observed upon strong stimulation, this can be equally well explained by endocytosis of surface-resident readily retrievable vesicles (Hua et al., 2011a) as well as recently released vesicles, both of which are present as intact patches. Assuming that the population of retrieved vesicle components was mixed on the level of the individual vesicles rather than on the level of vesicle populations is not necessary to explain the findings. Furthermore, these studies did not have the resolution to actually observe the behaviour of individual vesicle patches ( $~ 80 \mathrm{~nm}$ diameter) and their cohesion or disintegration and mixing upon retrieval. This makes the assertion that synaptic vesicles disintegrate upon fusion to the cell membrane pure conjecture. In conclusion, the data cited in support of the dispersal hypothesis is contentious and can be equally well explained by the cohesion hypothesis. For lipids, however, it seems clear that there is significant exchange with the cell membrane (Zenisek et al., 2002).

Cohesion hypothesis. The main arguments for the cohesion hypothesis come from experiments in which endogenous synaptic vesicle proteins were tagged with antibodies and observed with STED super-resolution fluorescence microscopy during physiological levels of stimulation. Antibody labelling of synaptotagmin 1 proteins on the cell surface revealed clusters of $\sim 80 \mathrm{~nm}$ in size (equivalent to the predicted diameter of a fully collapsed synaptic vesicle in the cell membrane; Willig et al., 2006). No stray proteins available for intermixing with recently exocytosed vesicles were evident. The same question can be addressed by blocking the epitopes of cell surface resident proteins with antibodies, sequentially triggering the release of vesicles while blocking retrieval, differentially labelling the vesicles released 
during each release sequence with antibodies, and detecting the degree of co-localization. This experiment was again performed for synaptotagmin 1, and only a fraction of the overlap that was observed during labelling with two differently labelled antibodies during the same release sequence could be observed in two-colour STED microscopy (Opazo et al., 2010). This again indicates little to no intermixing of vesicle proteins after fusion to the cell membrane. However, these experiments addressed the distribution of only one protein, synaptotagmin 1. A way to investigate the co-clustering of synaptic vesicle proteins is to block epitopes of a surface resident proteins with antibodies, then induce fusion of additional vesicles while blocking retrieval, labelling the new proteins on the surface, and investigating the degree of co-localization with another vesicle marker. When the two abundant synaptic vesicle proteins synaptotagmin 1 and synaptophysin are tagged in this way, and subsequently detected with two-colour STED microscopy, the two labels overlap just as much as a directly labelled primary antibody against synaptotagmin 1 and a labelled secondary antibody against the primary (Hoopmann et al., 2010). This high degree of colocalization observed at vesicle patch resolution ( $~ 80 \mathrm{~nm}$ patch size of vesicles fully collapsed into the membrane) indicates that vesicle proteins stay clustered on the cell membrane after release. I revisited here the data from Hoopmann et al. (2010) and further analysed the loss of synaptotagmin 1 from vesicle patches identified by synaptophysin staining (Figure 1.1). I found that a maximum of 3\% of synaptotagmin 1 proteins is lost from vesicle clusters (Figure 1.1C). This might even be an overestimation, as many of the spots might be identified as lost proteins from lack of co-localization with synaptophysin simply due to incomplete staining for synaptophysin (many "lost" spots are as bright as one would expect bona fide synaptic vesicle patches to be, and not dim as one would expect of individual lost proteins or broken patches; Figure 1.1A,B).

Supporting data for the cohesion hypothesis. There are numerous indirect lines of evidence that further support the cohesion hypothesis. First, several synaptic vesicle proteins have been observed to display a strong affinity for one another, thereby recruiting each other to vesicles. The best investigated example for this is the interaction of synaptobrevin/VAMP2 and synaptophysin, two of the most abundant synaptic vesicle proteins (Takamori et al., 2006; Wilhelm et al., 2014). They form complexes stable enough to isolate and observe in cryo-electron microscopy (Adams et al., 2015; Arthur and Stowell, 2007), and recruit each other to nascent synaptic vesicles (Becher et al., 1999; Mitter et al., 2003; Pennuto et al., 2003). Furthermore, it has recently been shown that the stoichiometry of $\sim 1: 2$ (synaptophysin vs. VAMP2) observed in electron microscopy (Adams et al., 2015; Arthur and Stowell, 2007) and quantitative analyses of synaptic vesicle protein content (Takamori et al., 2006; Wilhelm et al., 2014) is necessary for successful retrieval of synaptic vesicles from the cell membrane 
(Gordon et al., 2016), suggesting a stable complex. Other synaptic vesicle protein complexes consisting of SV2, VAMP2, synaptophysin, synaptotagmin 1, and the VATPase have been demonstrated and have even been shown to be resistant to detergent solubilisation (Bennett et al., 1992; also see Jia et al., 2006). Recent results on the ring-like clustering of calciumsensing proteins with $C 2 A B$ domains suggests that synaptotagmin 1 and Doc2A/B might be further candidates for the formation of stable complexes on the vesicle (Zanetti et al., 2016). It is difficult to imagine how such cohesive complexes could be disassembled during fusion of synaptic vesicles to the cell membrane. Second, synaptic vesicles are rich in cholesterol, compared to the cell membrane (Benfenati et al., 1989; Deutsch and Kelly, 1981; Michaelson et al., 1983; Takamori et al., 2006). Cholesterol is a major determinant of membrane rafts (Brown and Rose, 1992; Lingwood and Simons, 2010; Simons and Ikonen, 1997) and has been observed to organise membrane rafts in synapses (Chamberlain et al., 2001; Hering et al., 2003; Lang et al., 2001). It is thus reasonable to assume that it would also favour raft-like behaviour of synaptic vesicles after fusion to the cell membrane (Puchkov and Haucke, 2013; Rohrbough and Broadie, 2005). Direct observations of membrane rafts derived from synaptic vesicles are, however, still missing. Nevertheless, strong interactions between cholesterol and the synaptic vesicle protein VAMP2 as well as the synaptophysin/VAMP2 complex mentioned above have been described and proposed to be essential for synaptic vesicle biogenesis and budding (Mitter et al., 2003; Thiele et al., 2000). Third, synaptic vesicles seem to contain stable lumenal structural protein assemblies, which could well help to stabilize the fused vesicle patch further (Harlow et al., 2013). Fourth, the endocytic machinery with its adaptor proteins and molecular super-structures, such as clathrin coats, which must pre-assemble even before retrieval of the vesicle from the membrane (Hua et al., 2011a), could provide a scaffold that holds the vesicle together (Gimber et al., 2015). Fifth, synaptic vesicles do not only release as quanta (del Castillo and Katz, 1954), but also recycle as quanta (Gandhi and Stevens, 2003). Gandhi and Stevens (2003) expressed synaptopHluorin, a fusion construct of VAMP2 and the $\mathrm{pH}$-sensitive GFP variant pHluorin (Miesenböck et al., 1998), and followed the release and retrieval of single synaptic vesicle quanta by fluorescence microscopy. They observed that the fluorescence change upon vesicle fusion was always identical in size to the reciprocal fluorescence change upon vesicle retrieval. Since the surface resident synaptopHluorin molecules had been bleached in their experiments to increase the signal-to-noise ratio, no synaptopHluorins on the cell surface could contribute to the change in fluorescence upon vesicle retrieval. This means that the synaptopHluorin molecules that were retrieved were most likely the same ones that had been previously released. If there had been any significant intermixing of the previously released synaptopHluorins with the bleached cell surface resident synaptopHluorin molecules, this would have resulted in a smaller change in fluorescence upon retrieval than upon release. 
Sixth, the lifetimes of synaptic vesicle proteins, as measured in cultured neurons, are very similar (Cohen et al., 2013; Daly and Ziff, 1997; also see Figure 3.17), probably mostly within the measurement error of the techniques used to quantify them. This suggests that synaptic vesicle proteins age together, which is best explained by a cohesive synaptic vesicle ageing as a unit.

Taken together, these findings make a strong case that the synaptic vesicle stays intact during fusion to the cell membrane and subsequent retrieval. Accordingly, I will consider synaptic vesicles to be largely stable conglomerates of proteins and treat them as individual units throughout this Thesis. Further evidence for the cohesion hypothesis will be presented later during this Thesis, derived from experiments on the age and release behaviour of tagged vesicles that were followed for several days in hippocampal cultures (Figure 3.4; Figure 3.5, Figure 3.6, Figure 3.7).

A

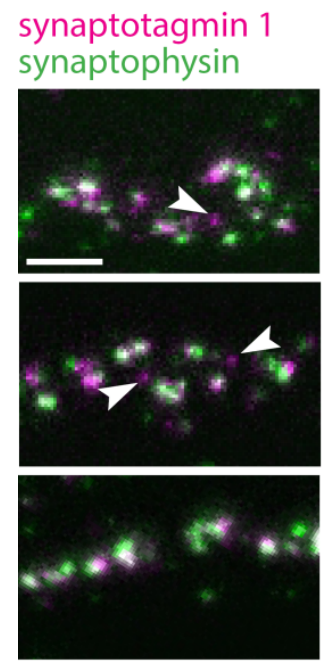

B

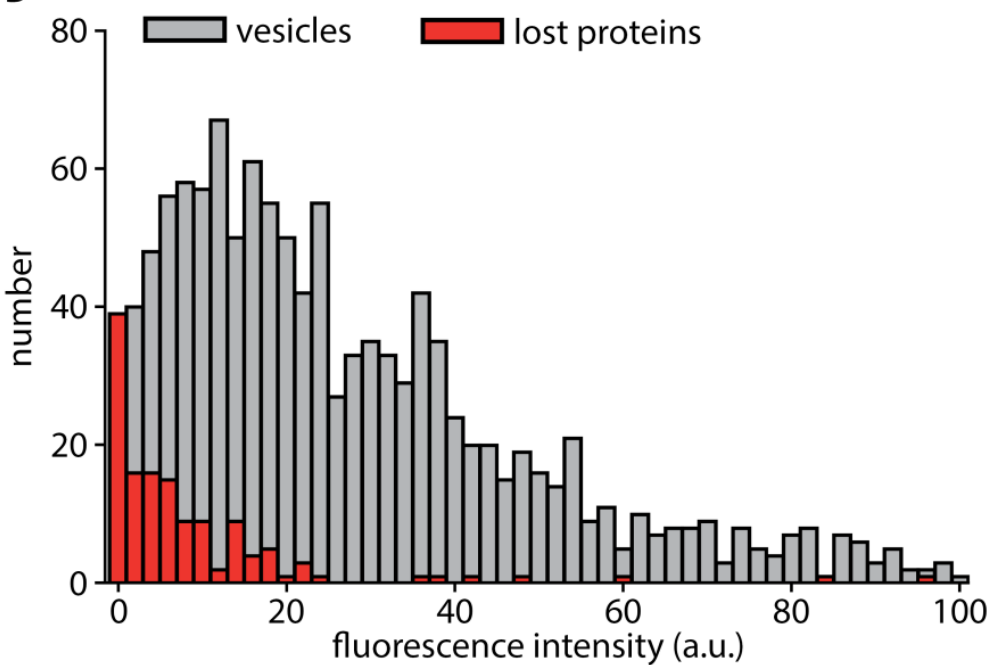

C

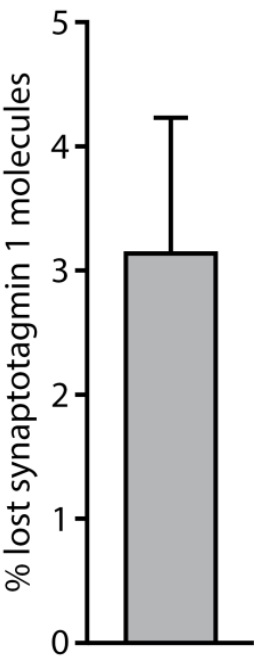

Figure 1.1: Synaptic vesicles maintain their cohesion upon fusion and only lose a minor fraction of their protein components.

(A) Exemplary images of a two-colour STED analysis of surface immunostaining for synaptotagmin 1 and synaptophysin. Prior to induced vesicle fusion, the surface epitopes of synaptotagmin 1 were blocked with unlabelled antibodies. Fusion was then allowed to proceed, while blocking endocytosis. Synaptotagmin 1 proteins on vesicles fused after blocking were then identified with a directly labelled primary antibody, while synaptophysin was detected with a primary/secondary pair of antibodies. Lost synaptotagmin 1 proteins (arrows) can then be identified. Note that this experimental design allows only the identification of lost synaptotagmin 1 proteins, as only the proteins on vesicles fused after the blocking step will be labelled, while all synaptophysin will be labelled, not just the proteins fused after the blocking step for synaptotagmin 1.

(B) Distribution of spot intensity of the synaptotagmin 1 immunostaining. Vesicles were identified by their co-localization with synaptophysin, lost proteins were identified by their lack of co-localization $(n=$ 3 independent experiments; 1219 vesicle spots and 136 lost protein spots quantified). 
(C) Quantification of the loss of synaptotagmin 1.

Note: the data presented here is a re-analysis of experiments published in Hoopmann et al. (2010). All data represent the mean \pm SEM.

\subsection{Synaptic vesicle pools or populations}

Based on their functional characteristics, synaptic vesicles have traditionally been sorted into three distinct pools (Denker and Rizzoli, 2010; Rizzoli and Betz, 2005; also see Figure 1.2): the readily releasable pool, which consists of only $\sim 1 \%$ of synaptic vesicles that are primed for release and respond immediately to stimulation, the recycling pool of $\sim 5-20 \%$ of synaptic vesicles that are able to release and recycle repeatedly during moderate, physiological levels of stimulation, and the reserve pool of $-80-95 \%$ of synaptic vesicles that do not respond to physiological levels of stimulation but can, at least partially, be released by severe, supraphysiological stimulation. Different terminologies have been advanced previously and also in recent years (Alabi and Tsien, 2012) to describe essentially the same phenomena.

A

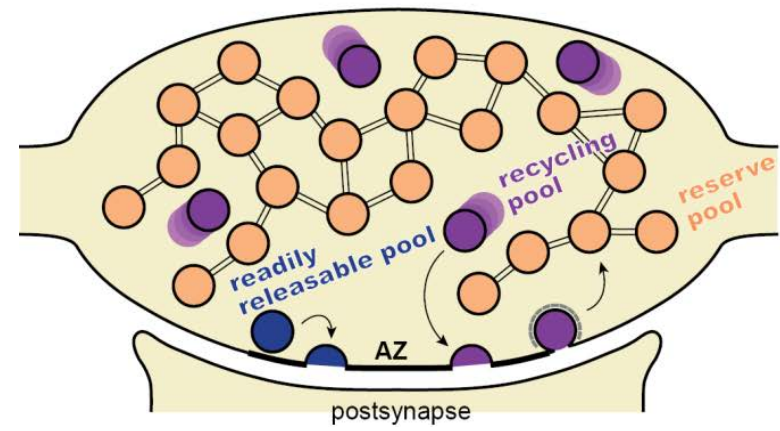

$\mathrm{B}$

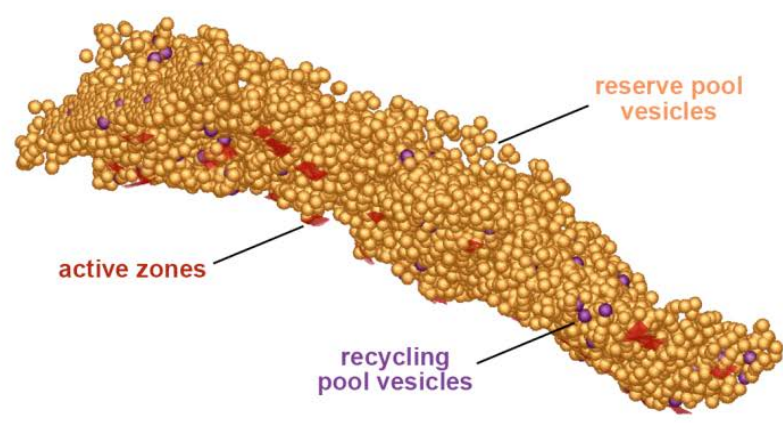

Figure 1.2: The three classical functionally distinct synaptic vesicle pools (adapted from Truckenbrodt and Rizzoli, 2015).

(A) Schematic illustrating the characteristics of the three classical synaptic vesicle pools: the readily releasable pool (dark blue) consists of synaptic vesicles primed for release at the active zone (AZ). The recycling pool (purple) consists of mobile vesicles that are quickly recruited for release after the readily releasable pool has been exhausted. These vesicles recycle several times during prolonged stimulation (grey bars on recycling vesicle: clathrin-coat). The reserve pool (orange) consists of largely immobile vesicles, tethered together and to the actin cytoskeleton by synapsin (grey connecting lines), and reluctant to release even under severe supra-physiological stimulation.

(B) An electron microscopy 3D reconstruction of synaptic vesicles labelled in situ in a zebrafish neuromuscular junction with FM-dyes during $2 \mathrm{~h}$ of free behaviour, followed by photoconversion (adapted from Denker et al., 2011a). Active zones are indicated in red, recycling pool vesicles that took up the dye during release and recycling are indicated in purple (note that only $\sim 1 \%$ of all vesicles released), reserve pool vesicles are indicated in orange. 
Readily releasable pool. The vesicles of the readily releasable pool can be considered to be a sub-population of the recycling pool. These are the recycling pool vesicles that are currently docked at the active zone and primed for release (Schikorski and Stevens, 2001). They are the first response group of synaptic vesicles that can immediately release upon stimulation (Delgado et al., 2000; Elmqvist and Quastel, 1965; Neves and Lagnado, 1999). It has to be noted, however, that not all docked vesicles can automatically be considered primed and releasable (Rizzoli and Betz, 2004; Xu-Friedman et al., 2001). Usually, less than $1-2 \%$ of all synaptic vesicles at a synaptic bouton are part of the readily releasable pool (Truckenbrodt and Rizzoli, 2015). Consequently, this pool is exhausted within only a few action potentials at high-frequency stimulation, often less than 10-20 (Delgado et al., 2000; de Lange et al., 2003; Richards et al., 2003; Stevens and Williams, 2007).

Recycling pool. If stimulation continues after the readily releasable pool has been exhausted, further vesicles will be recruited from the recycling pool. The release kinetics of these vesicles are usually slower (for a particularly striking example, see Neves and Lagnado, 1999). The recycling pool can sustain release during physiological levels of stimulation by retrieving released vesicles by endocytosis and refilling them with neurotransmitter (Südhof, 2004). This principle has been observed in virtually every animal and type of synapse under investigation (Harata et al., 2001a; Kuromi and Kidokoro, 1998; de Lange et al., 2003; Richards et al., 2003), and depending on the synapse type, $\sim 5-20 \%$ of synaptic vesicles are available for this type of release. Notably, in freely behaving animals, the size of the recycling pool is smaller than in artificially stimulated synapses, only $\sim 1-5 \%$ (Denker et al., 2011a; Körber et al., 2012; Figure 1.2B). The relative size of the recycling pool further seems to be inversely correlated to the number of synaptic vesicles per synapse. In small central synapses, such as those of hippocampal neurons, the recycling pool can comprise $-20-25 \%$ of all internalized synaptic vesicles or even up to $100 \%$ in particularly small synapses with $\sim 50$ or less vesicles (Marra et al., 2012; Rose et al., 2013).

Reserve pool. The rest of the synaptic vesicles at a synapse, $\sim 80-95 \%$, do not release at all during moderate levels of stimulation. Since they can be made available for release during high levels of stimulation, these vesicles have traditionally been termed reserve pool (Denker and Rizzoli, 2010; Rizzoli and Betz, 2005). They can, however, not be considered to be a true functional reserve, as the levels of stimulation necessary to release them can only be achieved in vitro and sometimes are several orders of magnitude higher than those reached in freely behaving animals (Denker et al., 2011a; Truckenbrodt and Rizzoli, 2015). For example, at least 5-10 Hz in frog neuromuscular junctions (Heuser and Reese, 1973; Richards et al., 2000) or $30 \mathrm{~Hz}$ in Drosophila larva neuromuscular junctions (Kuromi and 
Kidokoro, 2000) are necessary to recruit vesicles from this pool. The reserve pool vesicles further do not seem to exchange with the recycling pool vesicles at all, or only at a very low and slow rate (Richards et al., 2000). It might thus be more accurate to term this pool the resting pool (Alabi and Tsien, 2012). It has been proposed that these vesicles serve as a buffer for soluble synaptic proteins that act as co-factors of release or recycling (Denker et al., 2011b; Wragg et al., 2013). However, it is still largely unclear what distinguishes a recycling pool vesicle from a reserve pool vesicle on the molecular level (see Chapter 1.4).

Non-classical synaptic vesicle pools. Other pools have been described: the spontaneous pool of synaptic vesicles releasing in the absence of stimulation (Fatt, and Katz, 1952; Fatt and Katz, 1950; Figure 1.3A), the surface pool or readily retrievable pool of synaptic vesicles residing on the cell membrane (Gandhi and Stevens, 2003; Hua et al., 2011a; Figure 1.3B), and the super-pool of synaptic vesicles migrating between synapses (Darcy et al., 2006; Staras et al., 2010; Welzel et al., 2011; Figure 1.3C). Unlike the previously discussed pools, these pools are not strictly delineated along functional lines of division in terms of their response to evoked stimulation, with the arguable exception of the surface or readily retrievable pool. Nonetheless, these pool concepts are fairly well characterized and some of them can be integrated into the classical three-pool model discussed above, while others lie in parallel to it (Figure 1.4; Denker and Rizzoli, 2010; Truckenbrodt and Rizzoli, 2014, 2015).

A

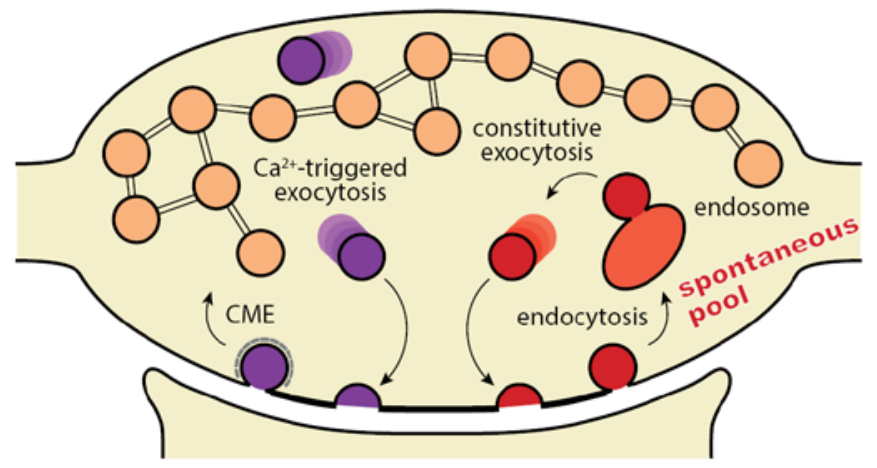

C

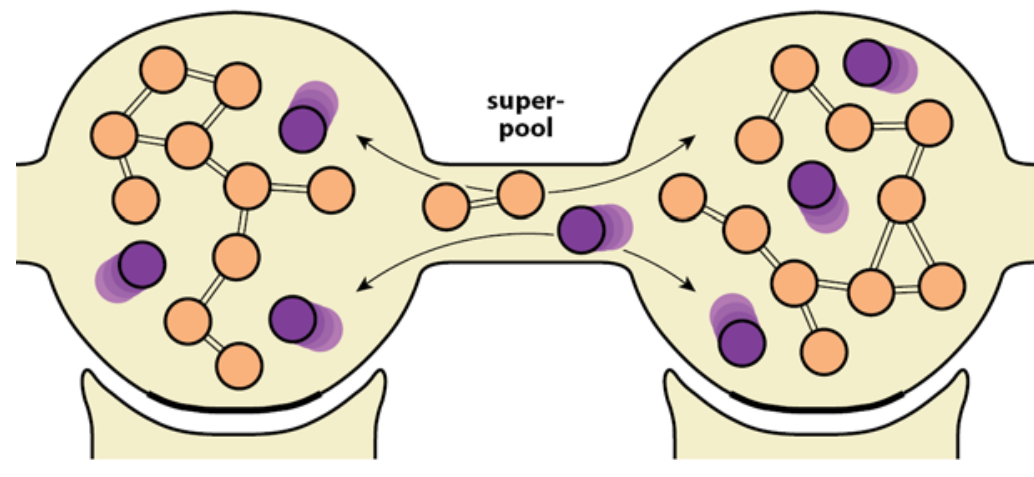

B

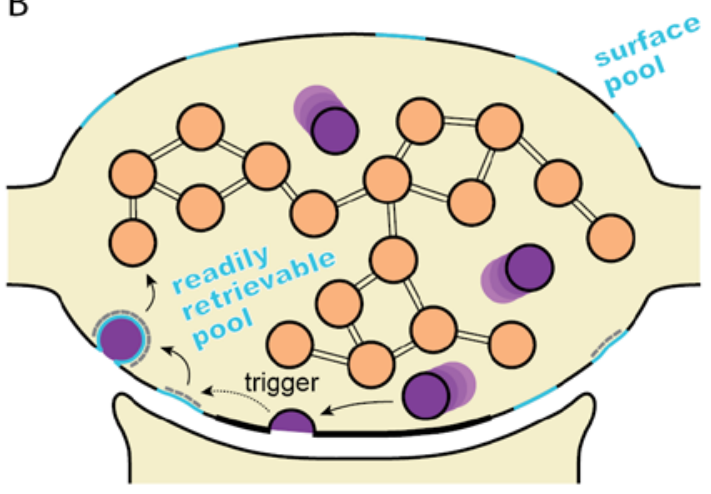

Figure 1.3: The three major nonclassical synaptic vesicle pools (adapted from Truckenbrodt and Rizzoli, 2015; colour-coding and symbology as in Figure 1.2).

(A) The spontaneous pool (red) undergoes constitutive exocytosis that is independent of evoked release. These vesicles might 
represent a separate pathway of release during synapse and vesicle maturation or maintenance (Truckenbrodt and Rizzoli, 2014), indicated by the endosome (light red).

(B) The surface pool (cyan) consists of synaptic vesicles residing on the cell membrane $(\sim 20 \%$ for most synaptic vesicle proteins, see Table 1.1). These vesicles are also readily retrievable in response to stimulation (Hua et al., 2011a), presumably as a compensatory mechanism for vesicle release.

(C) The super-pool consists of recycling pool and reserve pool vesicles that are dynamically exchanged between neighbouring synapses.

Spontaneous pool. The spontaneous pool (Figure 1.3A) consists of vesicles that release in the absence of any action potential or other apparent stimulus. Whether the same vesicles that behave in this way can also participate in evoked release remains controversial. Using largely equivalent, and indeed sometimes identical, experimental approaches, some labs found that spontaneously releasing vesicles can also respond to stimulation (Groemer and Klingauf, 2007; Hua et al., 2010, 2011b; Wilhelm et al., 2010), while others found that spontaneously releasing vesicles are incapable of evoked release and vice versa (Chung et al., 2010; Fredj and Burrone, 2009; Mathew et al., 2008; Sara et al., 2005). These conflicting results have puzzled researchers in the field. To solve the controversy and unify the published data, I recently suggested that all vesicle precursors might release constitutively as part of their final maturation steps at the synapse, before they become bona fide synaptic vesicles that release in response to stimulation or spontaneous local calcium fluctuations (Truckenbrodt and Rizzoli, 2014). The conflicting results obtained by the above studies might have arisen because of the use of neurons at different maturation stages, with different prevalence of early and late maturation vesicles. Immature neurons with a high prevalence of young, constitutively releasing vesicles might give the impression of two distinct pools. Whether this hypothesis will hold true will require further experimentation.

Surface pool. The surface pool (Figure 1.3B) consists of vesicles that are interpreted either as "stranded" on the cell membrane (Gandhi and Stevens, 2003), or more commonly as vesicles that are readily retrievable to compensate for release of internalized vesicles (Gandhi and Stevens, 2003; Hua et al., 2011a; Wienisch and Klingauf, 2006). For most major synaptic vesicle proteins, a fairly similarly large surface resident fraction has been described (Balaji and Ryan, 2007; Fernández-Alfonso et al., 2006; Granseth et al., 2006; Hoopmann et al., 2010; Revelo et al., 2014; Sankaranarayanan and Ryan, 2000; Wienisch and Klingauf, 2006; Table 1.1), so this does not seem to be an artefact of particular proteins missorted during vesicle cycling. This large readily retrievable surface population is presumably necessary to maintain a constant surface-to-volume ration of synapses during highfrequency release as well as to maintain a constant pool of recycling and releasable vesicles. The clathrin-mediated retrieval of a synaptic vesicle can take up to $\sim 30 \mathrm{~s}$ (Cocucci et al., 
2012), which necessitates a pool of readily retrievable vesicles for replenishment of the recycling pool, which would otherwise be exhausted before the recently released vesicles are retrieved. The readily retrievable pool can thus be considered part of the recycling pool (Figure 1.4).

Table 1.1: Surface resident fractions of major synaptic vesicle proteins and synaptic vesicleassociated proteins.

\begin{tabular}{|l|l|l|}
\hline protein & surface fraction & references \\
\hline $\begin{array}{l}\text { synaptobrevin/ } \\
\text { VAMP2 }\end{array}$ & $\sim 13-33 \%$ & $\begin{array}{l}\text { Granseth et al., 2006; Hoopmann et al., 2010; } \\
\text { Revelo et al., 2014; Sankaranarayanan and } \\
\text { Ryan, 2000; Wienisch and Klingauf, 2006 }\end{array}$ \\
\hline synaptophysin & $\sim 8-19 \%$ & Granseth et al., 2006; Revelo et al., 2014 \\
\hline synaptotagmin 1 & $\sim 19-24 \%$ & $\begin{array}{l}\text { Fernández-Alfonso et al., 2006; Opazo et al., } \\
\text { 2010; Revelo et al., 2014; Wienisch and } \\
\text { Klingauf, 2006; also see Figure 3.2 }\end{array}$ \\
\hline VGlut1/2 & $\sim 2-12 \%$ & Balaji and Ryan, 2007; Revelo et al., 2014 \\
\hline synapsin I/II & $\sim 14 \%$ & Revelo et al., 2014 \\
\hline Rab3 & $\sim 23 \%$ & Revelo et al., 2014 \\
\hline Vti1a & $\sim 8 \%$ & Hoopmann et al., 2010 \\
\hline
\end{tabular}

Super-pool. The super-pool concept (Figure 1.3C) was introduced to characterize the frequent exchange of synaptic vesicles between neighbouring synapses. This includes both recycling pool vesicles (Darcy et al., 2006; Staras et al., 2010; Welzel et al., 2011) and reserve pool vesicles (Fernandez-Alfonso and Ryan, 2008; Kamin et al., 2010). This shortdistance transport of synaptic vesicles seems to be mainly mediated by dynamic modulation of the actin cytoskeleton (Darcy et al., 2006) rather than the microtubule network. This exchange could be useful for pre-synaptic plasticity (Darcy et al., 2006; Staras et al., 2010) and for providing an extra-synaptic buffer of releasable synaptic vesicles lying in wait for periods of sudden local increases in demand for release (Staras et al., 2010).

Terminology of this Thesis. For the purpose of this Thesis, I will group the pools described here as follows, based on their functional commonalities in synaptic release (Figure 1.4). The recycling pool, the readily releasable pool, and the surface or readily retrievable pool have in common that they are actively involved in the evoked release of neurotransmitter. Vesicles sorted into any of these pools can be considered functionally identical on that level. The divisions of the above pools only characterize different stages in the synaptic vesicle cycle of priming, fusion, and recycling (Südhof, 2004). Accordingly, I will describe all vesicles from the recycling pool, the readily releasable pool, and the surface or readily retrievable pool as the releasable population of synaptic vesicles throughout this Thesis. In contrast, I will describe 
all other synaptic vesicles as the inactive population throughout this Thesis. The division between releasable and inactive synaptic vesicles is the major functionally and quantitatively relevant division of these organelles in the synapse and the data presented in this Thesis deals mainly with the characterization of this division and the transition of vesicles from the releasable state to the inactive state. The reasons for this division have so far been poorly characterized and are still largely unexplained, as will be discussed in the following Chapter.

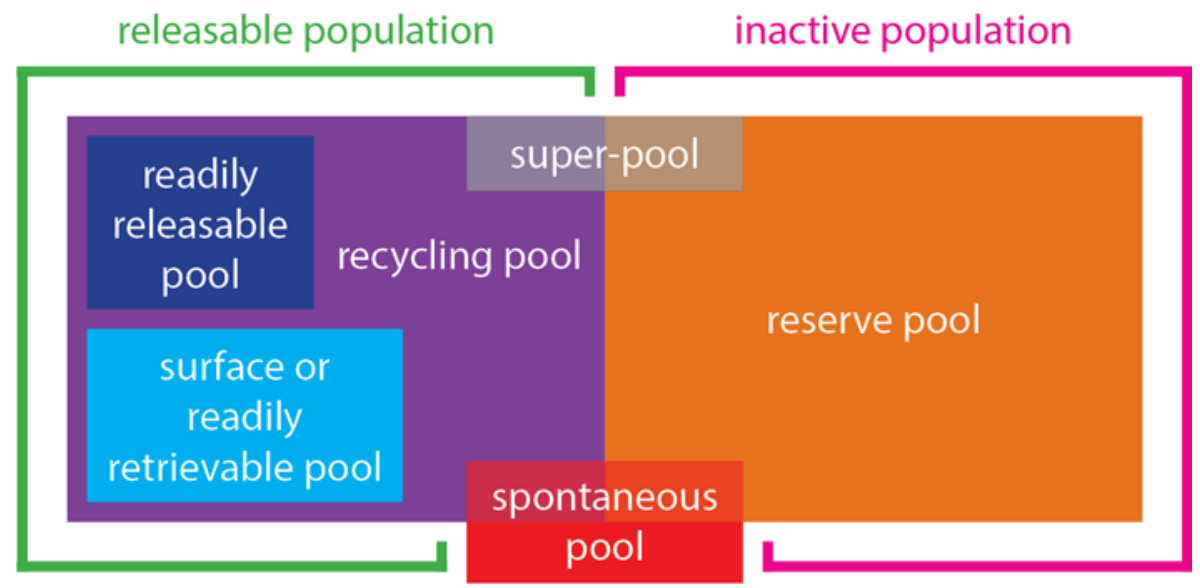

Figure 1.4: The distinctions and commonalities of synaptic vesicle pools.

The readily releasable pool (dark blue) and the surface or readily retrievable pool (cyan) can be considered sub-pools of the recycling pool (purple). They merely represent different stages of the synaptic vesicle cycle: readily releasable vesicles are primed, surface or readily retrievable vesicles have been fused to the cell membrane and are ready to be endocytosed and recycled, and the other recycling pool vesicles are prepared to participate in release on demand. These pools are thus grouped together as the releasable population (green) throughout this Thesis. In contrast, the reserve pool (orange) consists of synaptic vesicles that do not respond to stimulation and are thus referred to as the inactive population (pink) throughout this Thesis. The spontaneous pool (red) also does not respond to stimulation, but it is here regarded as a completely separate population of constitutively releasing vesicles that are not necessarily bona fide synaptic vesicles at all (see Chapter 1.2 and discussion in Truckenbrodt and Rizzoli, 2014), although some reports characterize the spontaneously releasing vesicles as sub-populations of the recycling pool or the reserve pool (reflected here in the partial overlap). The super-pool (grey) of vesicles that are exchanged between neighbouring synapses consists of vesicles from both the recycling pool and the reserve pool. Note that the relative sizes of the boxes do only loosely correspond to the actual relative pool sizes.

\subsection{Population identity and functional transitions}

The main focus of this Thesis will be how the distinction between releasable synaptic vesicles and inactive synaptic vesicles is achieved, maintained, and modulated. This problem can be broken down into several open questions in the field of synaptic physiology. What exactly distinguishes releasable synaptic vesicles from inactive synaptic vesicles? Can synaptic vesicles change their functionality during their life cycle, and if so, what triggers the 
transition into a different functional state? How can functional transitions be modulated? Why are there different functional states at all, and why do they appear to be controlled so tightly? None of these questions could be answered conclusively after more than six decades of research. Releasable synaptic vesicles do not have a privileged position in the synapse (Denker et al., 2009, 2011a; Rizzoli and Betz, 2004; Xu-Friedman et al., 2001), their ultrastructure is no indication (Harlow et al., 2013), the greater mobility of releasable vesicles (Kamin et al., 2010) ultimately remains unexplained and is rather another hallmark of the releasable population rather than an explanation of their distinct behaviour, and data on differences in protein composition between releasable and inactive synaptic vesicles are largely absent (but see Bal et al., 2013; Hua et al., 2011b; Raingo et al., 2012; Ramirez et al., 2012; discussed below). What little is known regarding these aspects, which all could potentially impact functional transitions of synaptic vesicles, will be laid out in this Chapter.

Localization. The subcellular localization of releasable synaptic vesicles is not a privileged one. Most of them are interspersed throughout the vesicle cluster with inactive vesicles (see for example Denker et al., 2009, 2011a; Rizzoli and Betz, 2004; Xu-Friedman et al., 2001; also see Figure 1.2B). This observation holds true across a wide variety of preparations, e.g. Drosophila larva neuromuscular junctions (NMJs; Denker et al., 2009), Drosophila larva central synapses (Denker et al., 2011a), locust NMJs (Denker et al., 2011a), the cricket optic lobe (Denker et al., 2011a), zebrafish NMJs (Denker et al., 2011a), mouse NMJs (Denker et al., 2011a), garter snake NMJs (Teng and Wilkinson, 2000), C. elegans NMJs (Denker et al., 2011a), embryonal chicken NMJs (Denker et al., 2011a), goldfish bipolar nerve terminals (Paillart et al., 2003), mammalian hippocampal synapses (Harata et al., 2001a, 2001b), and the mammalian Calyx of Held (de Lange et al., 2003). The readily releasable vesicles are by definition tethered at the active zone, but not all vesicles in this privileged position are also readily releasable (Rizzoli and Betz, 2004; Xu-Friedman et al., 2001). The localization of synaptic vesicles is thus no clear indication as to their functional status.

Mobility. It can be noted, however, that releasable synaptic vesicles that are not currently tethered at the active zone through priming are much more mobile than inactive vesicles (Denker and Rizzoli, 2010; Kamin et al., 2010). This is presumably necessary for them to navigate through the synaptic vesicle cluster upon stimulation to reach the active zone on demand (Rizzoli, 2014). The immobility of inactive synaptic vesicles is caused by synapsin, a super-abundant (Wilhelm et al., 2014) soluble protein that tethers synaptic vesicles to each other (Benfenati et al., 1989) and to the actin cytoskeleton (Greengard et al., 1993; Pieribone et al., 1995). A triple knock-out of all synapsin isoforms leads to an increased mobility of synaptic vesicles, including increased super-pool mobility between synapses (Fornasiero et 
al., 2012; Orenbuch et al., 2012), and a recognition-motif for the synaptic vesicle membrane curvature in the sequence of synapsin has been identified as the mediator of binding to synaptic vesicles (Krabben et al., 2011), confirming the "synapsin-hypothesis" of synaptic vesicle tethering (Greengard et al., 1993). The anchoring of inactive vesicles in the synapsin meshwork is dynamic and regulated by calcium-dependant phosphorylation (reviewed by Cesca et al., 2010; Fornasiero et al., 2010; Valtorta et al., 2011). This is most likely the mechanism of reserve pool mobilisation in response to high-frequency stimulation (Denker and Rizzoli, 2010; Gitler et al., 2008; Hilfiker et al., 1999; Rizzoli, 2014; Rizzoli and Betz, 2005; Song and Augustine, 2015). This dynamic modulation is mediated by the kinase CDK5, which decreases mobilization, and the phosphatase calcineurin, which facilitates mobilization (Kim and Ryan, 2010; Verstegen et al., 2014); MAP kinases and CaM kinases have also been implicated in this process (Cesca et al., 2010). While the mechanisms of synaptic vesicle immobilization are thus quite well understood, it remains completely unknown why some synaptic vesicles are tethered in the synapsin network and others are not. Activity-regulated phosphorylation states of synapsin surely play a role, but this seems to be a rather global and unspecific regulator of synaptic vesicle mobilization that does not target specific vesicles (Cesca et al., 2010; Fornasiero et al., 2012; Valtorta et al., 2011). Collapse of vesicles into the cell membrane most likely plays an important role in regulating synapsin binding, as removal of the synaptic vesicle membrane curvature sheds synapsin (Krabben et al., 2011). Assuming that synapsin-tethering is not an immediate occurrence and necessitates the step-wise binding of several synapsin proteins per synaptic vesicle (synapsin is the most abundant soluble protein at the synapse, with more than 60 copies per synaptic vesicle; Wilhelm et al., 2014), fusing a vesicle partially covered with synapsin would remove this partial covering again and prevent immobilization. The synapsin tethering could only proceed uninterrupted if the vesicle becomes non-releasable at some point, for whatever reason, ultimately resulting in immobilization. This suggests that synapsin tethering is just an effect of synaptic vesicle inactivation, and not necessarily its cause. Vesicles that stop releasing frequently would have a higher probability of becoming permanently tethered in the immobile cluster of inactive vesicles because they cannot shed synapsin; it is thus possible that synaptic vesicle inactivation precedes tethering. This switch of cause-and-effect perspective might be helpful in finally solving the pool conundrum (see Chapter 1.1).

Ultrastructure. There is very little data on synaptic vesicle ultrastructure, which could provide a hint on the difference between releasable and inactive synaptic vesicles. It has been observed that vesicles formed by compensatory endocytosis in direct response to stimulation are slightly larger in diameter than the average synaptic vesicle residing in the synapse (Watanabe et al., 2013a, 2013b). This could suggest that vesicle size is a 
determining factor in releasability. However, it remains unclear if the vesicles observed in these studies are bona fide synaptic vesicles. They might have closer correspondence to (pseudo-)endosomal compartments (Jähne et al., 2015), due to the unphysiological stimulation paradigms used in the above studies (Watanabe et al., 2013a, 2013b). They also have not conclusively been shown to be immediately releasable again, and they indeed seem to go through some clathrin-dependent steps of maturation, during which they shrink back to the average size of synaptic vesicles in the synapse before becoming releasable again (Watanabe et al., 2014). So vesicle size does not seem to be a reliable indicator of releasability. While synaptic vesicle size has been observed to differ between neurons in the same preparation as well as between preparations, changes in activity do not seem to impact synaptic vesicle size (Hu et al., 2008; Qu et al., 2009). Another study found that synaptic vesicle size is increased while releasability is decreased in synapses lacking the protein LAP, which participates in clathrin-mediated endocytosis (Zhang et al., 1998). However, this is both most likely a direct effect of decreased replenishment of the releasable population and defects in synaptic vesicle protein sorting due to deficient clathrin-mediated endocytosis (Zhang et al., 1998), rather than an effect of a reduction in releasability that can be directly attributed to vesicle size. While these data do not definitively exclude that synaptic vesicle size could have an impact on releasability, changes in synaptic vesicle diameter usually seem to be the side-effect of other processes and never the cause for changes in releasability; these larger organelles might indeed not be bona fide synaptic vesicles at all, but (pseudo-)endosomal structures arising from an overstrained endocytosis machinery in the wake of supra-physiological stimulation.

A recent study showed, through painstaking analysis of electron microscopy tomograms, that there is an electron-dense consensus structure inside synaptic vesicles (Harlow et al., 2013). This is a very interesting observation, as it suggests that the abundant protein complement of the synaptic vesicle (Takamori et al., 2006; Wilhelm et al., 2014) is not randomly arranged and does not differ much between vesicles; otherwise the intra-vesicular structure could not be repeated so faithfully between vesicles. However, the arrangement of this consensus structure seems to be indistinguishable between release-ready vesicles tethered at the active zone and vesicles that reside in the vesicle cluster further away from the active zone. The only difference is that the tethered vesicles are all oriented in the same direction. This suggests an important role of the protein arrangement on synaptic vesicles for release, a notion that is also implied by other observations of protein arrangements on synaptic vesicles (Adams et al., 2015; Arthur and Stowell, 2007; Zanetti et al., 2016), but it does so far not help in identifying a defining structural factor that distinguishes releasable and inactive synaptic vesicles. 
Molecular tags. The most likely parameter that could influence synaptic vesicle releasability is its quantitative molecular composition. While the synaptic vesicle is arguably the best characterized organelle to date, with its protein complement well described qualitatively and quantitatively by different complementary approaches (Blondeau et al., 2004; Burré et al., 2006a, 2006b; Morciano et al., 2005; Mutch et al., 2011; Takamori et al., 2006; Wilhelm et al., 2014), information on molecular differences between releasable and inactive vesicles is still scarce. While the synaptic vesicle appears to largely maintain its molecular makeup during recycling (Chapter 1.2), large variability in copy numbers between vesicles has been tentatively described for some particularly abundant proteins: synaptogyrin, synaptophysin, and synaptobrevin/VAMP2 (Mutch et al., 2011). It has to be noted, however, that the technique used by Mutch et al. (2011) might be prone to artefacts in protein copy number measurements, particularly for highly abundant proteins. They used an elegant TIRF-based setup to measure variations in immunostaining fluorescence between isolated single synaptic vesicles separated in microfluidic channels. It is unlikely that all epitopes can be detected by antibodies, due to epitope masking, mainly through steric hindrance (Fornasiero and Opazo, 2015; Opazo et al., 2012). This means that this detection method would systematically underestimate copy numbers. This approach would thus probably still yield relatively accurate numbers for low-abundance proteins, but high-abundance proteins will show a higher variation. Indeed, out of seven proteins analysed by Mutch et al. (2011), they underestimated the copy number of six proteins by at least half, compared to Western blotting and mass spectrometry methods (Takamori et al., 2006; Wilhelm et al., 2014), which do not suffer from this restriction, while the closest match in copy numbers was obtained for the least abundant proteins. Additionally, Mutch et al. (2011) did not distinguish between synaptic vesicles from different types of synapses but used a mixed preparation, which might again contribute to variation. So it remains unclear to what degree copy numbers can vary between synaptic vesicles. Another study tried to analyse differences in the protein complement of synaptic vesicles tethered to the plasma membrane (and fused to it), and vesicles of the vesicle cluster, not tethered to the plasma membrane (Morciano et al., 2005), but they did not arrive at quantitative distinctions. Yet other studies have suggested that small changes in protein makeup are possible during the synaptic vesicle life cycle (Burré et al., 2006b; Hoopmann et al., 2010).

Removing or adding only a few copies of specific proteins might be sufficient to modulate synaptic vesicle releasability. Some studies, employing pHluorin fusion constructs, have even claimed that specific protein markers can be used to distinguish synaptic vesicles of the spontaneous pool (Bal et al., 2013; Ramirez et al., 2012), an asynchronously releasing vesicle fraction (Raingo et al., 2012), and the reserve pool (Hua et al., 2011b). VAMP7 and 
Vti1a have both been identified as proteins localizing mainly to spontaneously releasing vesicles (Bal et al., 2013; Ramirez et al., 2012). This is in line with the recently formulated hypothesis that constitutive synaptic release might be mediated by organelles that are distinct from bona fide synaptic vesicles, and either "rejects" from the synaptic vesicle biogenesis pathway or early stages of it, or they might be more closely related to recycling endosomes (Truckenbrodt and Rizzoli, 2014; also see Chapter 1.3). VAMP4 has been implicated in mediating asynchronous release following several trains of high-frequency stimulation (Raingo et al., 2012). However, VAMP4 seems to predominantly participate in endocytosis rather than exocytosis following a single train of stimulation (Raingo et al., 2012). This again reinforces the notion of a pool of constitutively releasing vesicles related to endosomes and distinct from actual bona fide synaptic vesicles that can be characterized as releasable or inactive in the sense laid out in Chapter 1.3. There is only one study that claims a functional distinction between these two populations, based on the presence or absence of VAMP7 (Hua et al., 2011b). In this study, VAMP7 was found to localize to vesicles that do not respond well to stimulation, but that do release spontaneously. The same study also found that VAMP2-positive vesicles and VGlut-positive vesicles release spontaneously about equally well. This is again more in line with the identification of a separate spontaneous pool than identification of the reserve pool. This is supported by results from later studies on VAMP7 mentioned above (Bal et al., 2013; Ramirez et al., 2012) and by the fact that the pool of VAMP7-positive organelles found by Hua et al. (2011b) displayed an endocytosis defect compared to bona fide synaptic vesicles, compatible with delayed clathrin-mediated or nonclathrin endocytosis of endosomes. Also, the high cell surface resident fraction of VAMP7 found in this study, which exceeds the surface fraction of VGlut in the same study by 15fold, is not compatible with the notion that VAMP7 localizes specifically to the reserve pool. As laid out in Chapter 1.3, the surface pool is a part of the recycling pool and reserve pool markers should not be found there, especially not in excess of recycling pool markers (VGlut was used in this study to identify the recycling pool).

To summarise: no structural or molecular which distinguish releasable synaptic vesicles from inactive synaptic vesicles have been identified so far.

\subsection{The synaptic vesicle life cycle}

In search of the distinguishing factor of releasable and inactive synaptic vesicles, we need to consider the possibility that these are simply two states in the life cycle of the same vesicle, i.e. that a synaptic vesicle starts out as releasable and then becomes inactive, or vice versa, or that it alternates back and forth between these states. This is indeed a more likely scenario than that the neuron essentially produces two distinct types of organelles 
(releasable and inactive synaptic vesicles), both indistinguishable in all the parameters discussed in Chapter 1.4, but with fundamentally different behaviour. The fact that inactive synaptic vesicles can, at least to some degree (Denker and Rizzoli, 2010; Rizzoli and Betz, 2005), be mobilized by dynamic modulation of the synapsin meshwork (Cesca et al., 2010; Fornasiero et al., 2010; Valtorta et al., 2011) also argues for a scenario where releasable and inactive vesicles are distinct but connected stages in the vesicle life cycle. The final hint that prompted the investigation of this possibility in this Thesis came from the observation that vesicles that had been observed during release become inactive after a resting period (Richards et al., 2000, 2003; Rizzoli and Betz, 2004), and that mobile vesicles become immobile after a while (Kamin et al., 2010). These two observations seem to suggest a transition from the releasable state to the inactive state during the life cycle of the vesicle. The entire life cycle of the synaptic vesicle is, despite the fact that this is arguably the best characterized organelle of any cell type (Rizzoli, 2014; see Chapter 1.3 and Chapter 1.4), still relatively ill understood. I will nonetheless set out in this Chapter to construct a probable life cycle from the existing data, which will serve as a tentative framework for this Thesis (Figure 1.5). A the end of this chapter, I will restate the scope and aim of this thesis, which is to answer the open questions of the synaptic vesicle life cycle that are going to be raised here.

Biogenesis. Data on the biogenesis of synaptic vesicles is relatively scarce and diffuse, but a consensus picture emerges from data collected both from neurons and neuron-related cell types such as PC12 cells (Bonanomi et al., 2006; Rizzoli, 2014). How synaptic vesicle proteins are first brought together, to form a proto-vesicle patch in the ER or Golgi membrane, or a precursor vesicle, remains enigmatic. Based on data on protein assemblies formed by synaptophysin, VAMP2, and other synaptic vesicle proteins (Becher et al., 1999; Bennett et al., 1992; Jia et al., 2006; Mitter et al., 2003; Pennuto et al., 2003; Thiele et al., 2000; Zanetti et al., 2016), some (Rizzoli, 2014; Thiele et al., 2000) have suggested that synaptic vesicle micro-domains already start to organize co-translationally on the ER membrane or during initial sorting steps in the Golgi apparatus, aided by raft organization via cholesterol and, possibly, certain phospholipids (Jia et al., 2006; Thiele et al., 2000; Verstreken et al., 2009). Even if these early assembly steps do occur (see below for further discussion of the role of synaptophysin), further processing will be necessary to form a mature bona fide synaptic vesicle (reviewed by Bonanomi et al., 2006; Hannah et al., 1999; Prado and Prado, 2002; Rizzoli, 2014). Synaptophysin has been particularly well investigated for its role in synaptic vesicle biogenesis. In neuron-related PC12 cells, it passes from the ER to the Golgi apparatus to the cell membrane, from where it is endocytosed and recycled back and forth between endosomes and the cell membrane several times, before it ultimately arrives in synaptic vesicle-like organelles (Cameron et al., 1991; Johnston et al., 1989; 
Regnier-Vigouroux et al., 1991). A similar trafficking process is observed with the fluid-phase marker HRP in PC12 cells (Bauerfeind et al., 1993). In a process that can take several hours, HRP ultimately arrives from the cell surface via endosomes to synaptic vesicle-like organelles (Bauerfeind et al., 1993). How this trafficking process is recapitulated in neurons, and whether it takes place in the cell body or at the synapse, remains an open question. The main organizing sorting mechanism that brings together all the dozens of different types of proteins found on synaptic vesicles (Blondeau et al., 2004; Burré et al., 2006a; Takamori et al., 2006), and in the correct copy numbers no less (Takamori et al., 2006; Wilhelm et al., 2014), ultimately remains enigmatic. Synaptophysin and the related synaptogyrin have been proposed to be this mechanism, based on their function in synaptic vesicle protein clustering during endocytosis (Gordon et al., 2011; Kwon and Chapman, 2011; Stevens et al., 2013). This is supported by observations that synaptophysin triggers the assembly of synaptic vesicle-like organelles even in cell types that do not normally form such organelles (Johnston et al., 1989; Leube et al., 1989, 1994; but for an opposing view also see Cameron et al., 1991; Linstedt and Kelly, 1991; Regnier-Vigouroux et al., 1991). On the other hand, knockouts of synaptophysin and synaptogyrin have minimal phenotypes and synaptic vesicles are still formed (Eshkind and Leube, 1995; Mcmahon et al., 1996; Stevens et al., 2013), even in double knock-outs of synaptophysin and synaptogyrin (Abraham et al., 2006; Janz et al., 1999), casting doubt on this hypothesis. However, synaptophysin still seems to be the only protein that manages to consistently localize to synaptic vesicle-like organelles when exogenously expressed by itself (Feany et al., 1993), so a fundamental role of this protein in synaptic vesicle biogenesis is difficult to discount completely (Rizzoli, 2014). It should be noted, however, that there seem to be different types of synaptic vesicle precursors, carrying different proteins (Feany et al., 1993; Okada et al., 1995), which suggests that synaptic vesicle assembly is not achieved solely by protein-protein and protein-lipid interactions in the ER/Golgi pathway, but entails further maturation steps (Bonanomi et al., 2006; Rizzoli, 2014).

Anterograde transport. In whatever way synaptic vesicle precursors are first assembled in the secretory pathway of the cell body, the next step inevitably has to be the transport to the pre-synaptic fusion sites (reviewed by Hirokawa et al., 2010; Vale, 2003), since the presynapse itself is incapable of protein biosynthesis (Steward and Schuman, 2003; but for opposing views see Giuditta et al., 2002; Piper and Holt, 2013). This is accomplished by migration along the axonal microtubule network via motors such as KIF1A and KIF1B $\beta$ (Niwa et al., 2008; Okada et al., 1995; Zhao et al., 2001). These bind to synaptic vesicle precursors via their PH domains (Klopfenstein and Vale, 2004; Klopfenstein et al., 2002) and Liprin- $\alpha$ (Wagner et al., 2009) or, mediated by DENN/MADD, the synaptic vesicle trafficking protein Rab3 (Niwa et al., 2008); additional mechanisms, e.g. JNKs and ARL-8 (Wu et al., 2013), are 
likely involved. Two ill investigated, but nonetheless essential, issues in anterograde transport are: first, that synaptic vesicles need to be selectively gated at the axon initial segment, or pre-axonal exclusion zone, where dendritic cargo is retained and only axonal cargo is allowed to pass (Farías et al., 2015; Song et al., 2009), which is the basis of neuronal polarization (Craig and Banker, 1994; Kobayashi et al., 1992; Nakada et al., 2003; Winckler et al., 1999), and second, how it is achieved that young synaptic vesicle precursors are transported mainly anterogradely (while aged synaptic vesicles marked for degradation are only transported retrogradely, see below). Both issues are presumably solved through Rab3-mediated selection of the specific motor proteins for anterograde transport identified above (Kapitein and Hoogenraad, 2011; Song et al., 2009).

Maturation. How a fully functional bona fide synaptic vesicle is formed from the precursor organelles arriving at the synapse is particularly enigmatic. It remains somewhat controversial whether synaptic vesicle components are transported to the synapse on the same precursor organelle or on distinct carriers, but the latter view is best supported by the published data (Bonanomi et al., 2006; Feany et al., 1993; Okada et al., 1995; Rizzoli, 2014). For example, Feany et al. (1993) found synaptotagmin 1, synaptophysin, and SV2 sorted to distinct organelles after overexpression. This was, however, done in non-neuronal cell types, which does not exclude the possibility that the neuronal machinery of protein sorting would target these major synaptic vesicle proteins to the same precursor. Okada et al. (1995), however, analysed the association of the same synaptic vesicle proteins with the KIF1A motor of anterograde transport of synaptic vesicle precursors to the synapse in cultured neurons. They found that SV2 migrated on different carriers as synaptotagmin 1 and synaptophysin, separated by differential density centrifugation and immunoisolation. The different components need to be brought together, which presumably necessitates the fusion of the precursors to the same compartment, e.g. the cell membrane or synaptic endosomes, where the components are assembled into a synaptic vesicle, which is then formed by budding (Jähne et al., 2015; Rizzoli, 2014; Truckenbrodt and Rizzoli, 2014). That such processes can principally occur at the synapse is well documented (for recent examples, see Hua et al., 2011a; Watanabe et al., 2014), but their involvement in synaptic vesicle biogenesis ultimately requires further experimental investigation.

Activity and inactivity of mature vesicles at the synapse. It is unclear how long the maturation of the synaptic vesicle takes. It could then join either the releasable population or the inactive population. It is unclear, whether it preferentially joins either or whether it can switch between these two states, and how frequently this switch could take place. It has been speculated that synaptic vesicles start out in the active, releasable population, before 
they become inactive (Denker and Rizzoli, 2010; Rizzoli and Betz, 2005). This is largely conjecture at this point, based on observations that recently released synaptic vesicles seem to become immobile and inactive after a while (Kamin et al., 2010; Richards et al., 2000, 2003; Rizzoli and Betz, 2004). The same studies did not observe synaptic vesicles coming back from the inactive state. However, the above studies only observed synaptic vesicle dynamics on a timescale of minutes or a few hours at most. This is an insufficient time span to investigate possible future changes in releasability, as the entire synaptic vesicle life cycle takes, on average, at least 3-4 days in culture (Cohen et al., 2013; Daly and Ziff, 1997). In situ, where the studies of Richards et al. (2000, 2003) and Rizzoli \& Betz (2004) were performed, this is probably even longer (Price et al., 2010; Rosenberg et al., 2014; Eugenio F. Fornasiero, personal communication). It also remains unclear whether synaptic vesicles start their life cycle at the synapse in the active state, as the above studies did not employ a method of determining vesicle age.

Degradation. Once the synaptic vesicle becomes active, whether directly or after a resting period, and starts to participate in release, it presumably has a limited lifetime. Synaptic vesicle release and modulation of activity necessitates many protein-protein interactions, conformational changes, changes in $\mathrm{pH}$, post-translational modifications, and exposure to reactive oxygen species. All these events have the potential to damage the synaptic vesicle by unfolding or modifying proteins (Burré et al., 2006b; Dobson, 1999, 2003; Matouschek, 2003; Prakash and Matouschek, 2004; see Chapter 4.4 in the Discussion for an in-depth treatment of this aspect). Additionally, the synapse is metabolically extremely active (Harris et al., 2012; Laughlin et al., 1998; Rangaraju et al., 2014), which has the potential to damage synaptic vesicles via local generation of reactive oxygen species by mitochondria (Davies, 2005; Massaad and Klann, 2013). It is unclear how exactly synaptic vesicle degradation proceeds, and under which conditions. It has recently been suggested that local maintenance mechanisms, mediated by the skywalker protein, might remove and replace damaged components of synaptic vesicles locally (Uytterhoeven et al., 2011). It is unlikely, however, that this mechanism alone is sufficient for removal of ageing synaptic vesicle proteins. The most prevalent view is that ageing synaptic vesicles are fused locally to endolysosomal compartments (Haberman et al., 2012) or to autophagosomes (Binotti et al., 2014), or that multi-vesicular bodies are locally formed via the ESCRT pathway (Sheehan et al., 2016), which are then transported back to the cell body for final degradation (Rizzoli, 2014). This would solve the issue of the Rab3-mediated anterograde trafficking signal (see above), which seemingly persists also at the synapse (Rizzoli, 2014). To achieve retrograde transport, this signal needs to be masked, or anterograde trafficking will prevail and the ageing vesicle will remain at the synapse. Fusing the vesicle to endolysosomal 
compartments would dilute and possibly override the anterograde trafficking signal, and engulfing the vesicle in autophagosomes or multi-vesicular bodies would separate the signal from the anterograde motors via a lipid bilayer.

Open questions and aim of this Thesis. Clearly, several key aspects of the synaptic vesicle life cycle are poorly understood, particularly regarding timing and functional transitions. The purpose of this Thesis was to fill those gaps in our understanding of synaptic physiology, particularly with regard to quantifying timing in long-term temporal dynamics. These questions include: are there two distinct populations of releasable and inactive synaptic vesicles or can synaptic vesicles switch functionality? How much of the synaptic vesicle lifetime is spend in the releasable and in the inactive state? How often can a synaptic vesicle be released during its lifetime? What are the consequences of vesicle ageing and damage accumulation on functionality? Are there specific molecular differences between releasable and inactive synaptic vesicles and can those be modulated during the vesicle's lifetime? Many of these questions are not only important to understand the basic cell biological principles of neurotransmission, but may also impact on neurodegenerative diseases, which often are associated with changes in synaptic vesicle release. 


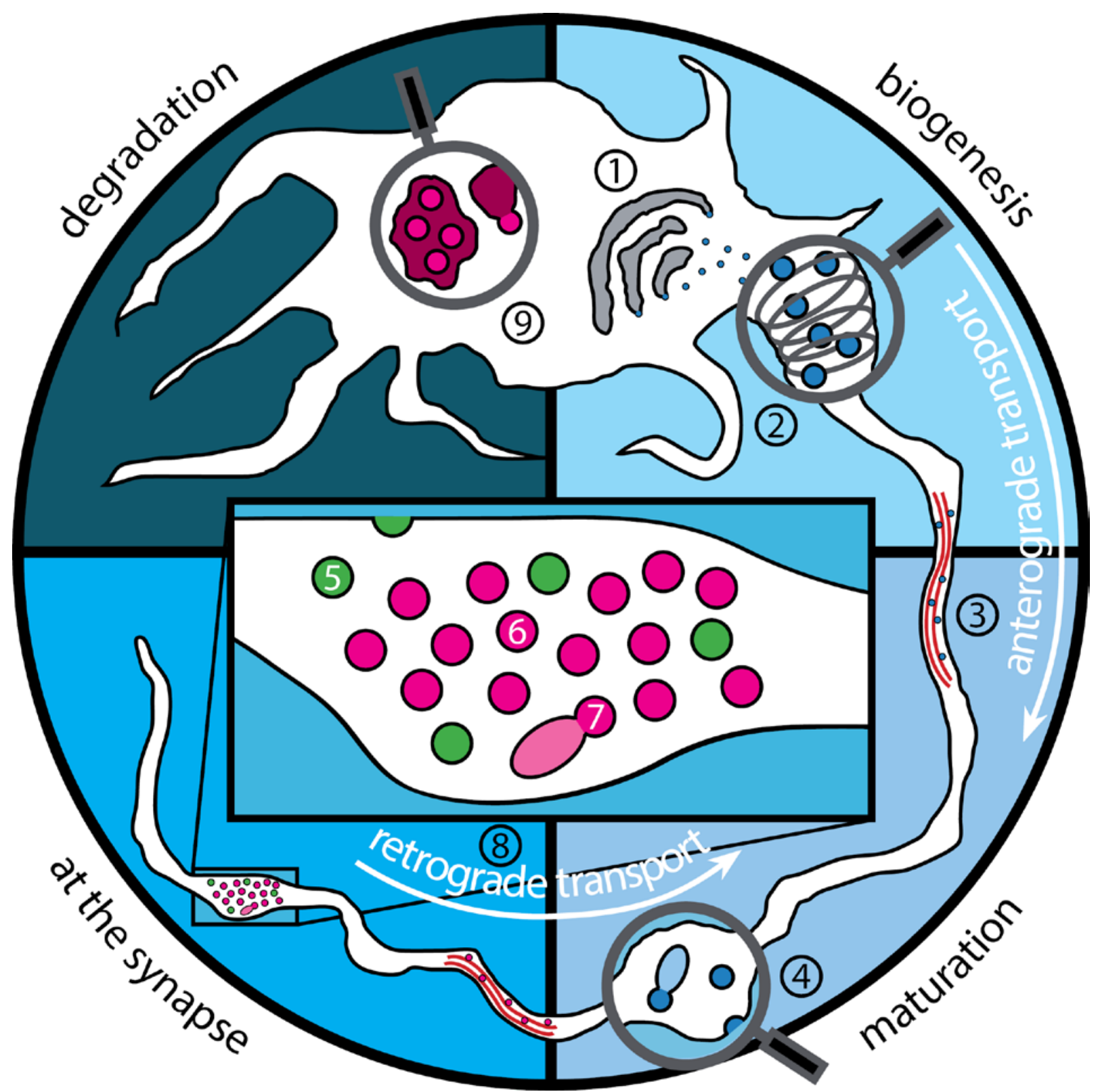

Figure 1.5: The synaptic vesicle life cycle.

Synaptic vesicle precursors (blue) are produced in the cell body, where they most likely arise from the ER, and are processed in the Golgi apparatus (grey sheets) (1). The precursors are then gated at the axon initial segment (grey meshwork) (2) and transported anterogradely along the axonal microtubule network (red) towards the synapses (3). Once the precursors reach a synapse, they have to undergo further maturation (4), possibly through repeated constitutive release and processing via endosomes (light blue), before they become active and responsive to evoked release (5). After a while, the releasable synaptic vesicles (green) might become inactive (pink) (6). This process as well as later stages of the synaptic vesicle life cycle might be modulated by local maintenance mechanisms via endosomes (light pink) (7). The synaptic vesicles might then at some later point be transported back (8) to the cell body for degradation in multi-vesicular bodies, autophagosomes, or lysosomes (dark pink) (9), although degradation could also be initiated locally (7). Note that the exact details of biogenesis (1) and degradation (8) of synaptic vesicles are still ill understood, and that the transition from activity (6) to inactivity (7) is conjecture at this point. 


\section{Materials and Methods}

\subsection{Hippocampal cultures}

Neuronal cultures were produced from rat hippocampi of P1-P2 rats in an adapted version of classical protocols (Banker and Cowan, 1977; Kaech and Banker, 2006). The rat pups were killed by decapitation, and the skulls opened to extract the brain. Subsequently, hippocampi were removed under a dissection microscope and collected in HBSS (Thermo Fisher Scientific, Waltham, MA, USA). The hippocampi were then digested in enzyme solution (Table 1 ) for $1 \mathrm{~h}$ to prepare the extraction of neuronal precursor cells. Following enzyme treatment, hippocampi were washed with Neurobasal-A (Thermo Fisher Scientific, Waltham, MA, USA), and subsequently incubated for $15 \mathrm{~min}$ in enzyme inactivating solution (Table 2), followed by another wash with Neurobasal-A and finally mechanical disruption. Cells were then counted and seeded at a concentration of $\sim 80,000 / \mathrm{cm}^{2}$ on $18 \mathrm{~mm}$ glass coverslips (Thermo Fisher Scientific, Waltham, MA, USA) that had been prepared by treatment with nitric acid (Sigma-Aldrich, St. Louis, MO, USA), followed by thorough washes with sterile water, sterilization, and coating with $1 \mathrm{mg} / \mathrm{ml}$ PLL (Sigma-Aldrich, St. Louis, MO, USA). The cells were allowed to adhere to the coverslips in plating medium (Table 3 ) for $1-4 \mathrm{~h}$ at $37^{\circ} \mathrm{C}$ in a $5 \% \mathrm{CO}_{2}$ atmosphere in the cell culture incubator. Afterwards, the medium was changed to culture medium (Table 4). Neurons were maintained in a cell culture incubator at $37^{\circ} \mathrm{C}$ in a $5 \% \mathrm{CO}_{2}$ atmosphere, generally for 14-21 days before use in experiments.

Table 2.1: Enzyme solution for isolation of neuronal precursor cells from rat hippocampi (solution equilibrated with carbogen for $10 \mathrm{~min}$ and sterile filtered before use).

\begin{tabular}{|l|l|l|}
\hline item & concentration & company \\
\hline DMEM & base solvent & Lonza, Basel, Switzerland \\
\hline L-cysteine & $0.5 \mathrm{mg} / \mathrm{ml}$ & Sigma-Aldrich, St. Louis, MO, USA \\
\hline $\mathrm{CaCl}_{2}$ & $100 \mathrm{mM}$ & Merck KGaA, Darmstadt, Germany \\
\hline EDTA & $50 \mathrm{mM}$ & Merck KGaA, Darmstadt, Germany \\
\hline papain & $2.5 \mathrm{U} / \mathrm{ml}$ & Cell Systems, Spich, Germany \\
\hline
\end{tabular}

Table 2.2: Enzyme inactivating solution for isolation of neuronal precursor cells from rat hippocampi.

\begin{tabular}{|l|l|l|}
\hline chemical & concentration & company \\
\hline DMEM & base solvent & Thermo Fisher Scientific, Waltham, MA, USA \\
\hline albumin & $0.2 \mathrm{mg} / \mathrm{ml}$ & Applichem, Darmstadt, Germany \\
\hline trypsin inhibitor & $0.2 \mathrm{mg} / \mathrm{ml}$ & Sigma-Aldrich, St. Louis, MO, USA \\
\hline FCS & $10 \%(\mathrm{w} / \mathrm{v})$ & Biochrom, Berlin, Germany \\
\hline
\end{tabular}


Table 2.3: Plating medium for seeding isolated hippocampal neuronal precursor cells on coverslips.

\begin{tabular}{|l|l|l|}
\hline chemical & concentration & company \\
\hline MEM & base solvent & Thermo Fisher Scientific, Waltham, MA, USA \\
\hline horse serum & $10 \%(w / v)$ & Biochrom, Berlin, Germany \\
\hline glucose & $3.3 \mathrm{mM}$ & Merck KGaA, Darmstadt, Germany \\
\hline glutamine & $2 \mathrm{mM}$ & Lonza, Basel, Switzerland \\
\hline
\end{tabular}

Table 2.4: Rat hippocampal neuron culture medium.

\begin{tabular}{|l|l|l|}
\hline item & concentration & company \\
\hline Neurobasal-A & base solvent & Thermo Fisher Scientific, Waltham, MA, USA \\
\hline B27 supplement & $1: 50$ & Thermo Fisher Scientific, Waltham, MA, USA \\
\hline GlutaMAX & $1: 100$ & Thermo Fisher Scientific, Waltham, MA, USA \\
\hline Pen/Strep & $1: 500$ & Lonza, Basel, Switzerland \\
\hline
\end{tabular}

\subsection{Live-cell tagging of synaptic vesicles with antibodies}

To tag recycling synaptic vesicles, I used a mouse monoclonal antibody, designated clone 604.2 (unconjugated \#105 311 or Atto647N-conjugated \#105 311AT1, Synaptic Systems, Göttingen, Germany), directed against the lumenal domain of synaptotagmin 1, or a rabbit polyclonal antibody directed against the lumenal domain of VGAT (\#131 103CpH, Synaptic Systems, Göttingen, Germany). Synaptotagmin 1 and VGAT are currently the only two synaptic vesicle proteins for which reliable antibodies are available for live-cell immunostainings (Kraszewski et al., 1995; Martens et al., 2008; Matteoli et al., 1992). Neurons were tagged with antibody diluted to $\sim 8.33 \mu \mathrm{g} / \mathrm{ml}$ (1:120 from a $1 \mathrm{mg} / \mathrm{ml}$ stock) for 1 $h$, unless otherwise indicated. Neurons were kept in their own culture medium throughout the entire procedure by placing them in a new well containing $300 \mu \mathrm{l}$ of their original culture volume of 1-1.5 ml and washed briefly 2-3 times with ice-cold Tyrode's solution (Table 5) to remove excess antibody before placing them back into the remaining medium. Tyrode's solution was kept ice-cold to suppress activity of the cultures during washing. Both measures were essential to prevent cell death induced by excitotoxicity caused by osmolarity shocks from fresh medium or medium from sister cultures.

Determining tagged vesicle populations. The different vesicle populations labelled with this tagging approach were determined by altering the labelling conditions (see Figure 3.2 for result). (1) Tagging living neurons for $1 \mathrm{~h}$ leads to labelling of the recycling pool (evoked release), the spontaneous pool (spontaneous release), and the surface pool. To differentiate between the recycling pool, the spontaneous pool, and the surface pool, vesicles were additionally tagged (2) in living neurons treated with 0.5-1 $\mu \mathrm{M}$ TTX (Cayman Chemical Company, Ann Harbor, MI, USA) to suppress evoked release (yields the spontaneous pool 
plus the surface pool), and (3) in living neurons treated with TTX and kept on $4^{\circ} \mathrm{C}$ (surface pool only, as the cold temperature inhibits any physiological reactions). Sequentially tagging neurons after fixation and permeabilization yields the total pool of vesicles in each of these experiments; this was done by cutting the cover slips in half and using only one half for sequential tagging and keeping the other half for comparison. This was necessary to determine the size of all vesicle pools assessed in these experiments relative to the total pool of vesicles to achieve comparability between experiments. All antibody incubations in these experiments were performed for $1 \mathrm{~h}$. To finally determine pools fractions, the fraction determined in (2) subtracted from the fraction determined in (1) yields the recycling pool fraction, and the fraction determined in (3) subtracted from the fraction determined in (2) yields the spontaneous pool fraction.

Validation of live-cell antibody-tagging. To determine how long the antibodies stay bound to their epitopes after tagging (see Figure 3.1D for result), I fixed and permeabilized neuronal cultures and performed an immunostaining with the 604.2-Atto647N antibody against the lumenal domain of synaptotagmin 1. I then cut the cover slips in half and post-fixed one half of the cover slips and immunostained for synaptophysin, as a marker of synapses and synapse size. I kept the other half of the cover slip for up to 10 days in PBS (Table 6$)+2.5 \%$ (w/w) BSA (Applichem, Darmstadt, Germany) + 0.05\% (w/w) $\mathrm{NaN}_{3}$ (Sigma-Aldrich, St. Louis, $\mathrm{MO}, \mathrm{USA})+10 \mu \mathrm{g} / \mu \mathrm{l}$ control peptide against which the antibody had been raised (\#105-1P, Synaptic Systems, Göttingen, Germany). Assuming a molecular weight of $150 \mathrm{kDa}$ for the mouse IgG antibodies (Feng and Konishi, 1992), and determining the molecular weight of the control peptide (first 19 amino acids of rat synaptotagmin 1) in silico as $\sim 1.85 \mathrm{kDa}$, the concentration of control peptide was chosen to be at least -100 -times in molar excess over the initial antibody concentration. Note that the actual excess was likely several fold higher, as not all antibody will have bound to epitopes.

Table 2.5: Tyrode's solution for washing steps of living neuronal cultures ( $\mathrm{pH}$ adjusted to 7.4 and sterile filtered before use).

\begin{tabular}{|l|l|l|}
\hline item & concentration & company \\
\hline $\mathrm{ddH}_{2} \mathrm{O}$ & base solvent & $\begin{array}{l}\text { from Lab Water Purification System from } \\
\text { Sartorius, Göttingen, Germany }\end{array}$ \\
\hline $\mathrm{NaCl}$ & $124 \mathrm{mM}$ & Sigma-Aldrich, St. Louis, MO, USA \\
\hline $\mathrm{KCl}$ & $5.0 \mathrm{mM}$ & Sigma-Aldrich, St. Louis, MO, USA \\
\hline glucose & $30 \mathrm{mM}$ & Merck KGaA, Darmstadt, Germany \\
\hline $\mathrm{HEPES}$ & $25 \mathrm{mM}$ & Merck KGaA, Darmstadt, Germany \\
\hline $\mathrm{CaCl}_{2}$ & $2 \mathrm{mM}$ & Sigma-Aldrich, St. Louis, MO, USA \\
\hline $\mathrm{MgCl}_{2}$ & $1 \mathrm{mM}$ & Sigma-Aldrich, St. Louis, MO, USA \\
\hline
\end{tabular}


Table 2.6: Phosphate buffered saline (PBS), pH adjusted to 7.3.

\begin{tabular}{|l|l|l|}
\hline item & concentration & company \\
\hline $\mathrm{ddH}_{2} \mathrm{O}$ & base solvent & $\begin{array}{l}\text { from Lab Water Purification System from } \\
\text { Sartorius, Göttingen, Germany }\end{array}$ \\
\hline $\mathrm{NaCl}$ & $137 \mathrm{mM}$ & Sigma-Aldrich, St. Louis, MO, USA \\
\hline $\mathrm{KCl}$ & $2.7 \mathrm{mM}$ & Sigma-Aldrich, St. Louis, MO, USA \\
\hline $\mathrm{Na}_{2} \mathrm{KPO}_{4}$ & $10 \mathrm{mM}$ & Sigma-Aldrich, St. Louis, MO, USA \\
\hline $\mathrm{KH}_{2} \mathrm{PO}_{4}$ & $2 \mathrm{mM}$ & Sigma-Aldrich, St. Louis, MO, USA \\
\hline
\end{tabular}

\subsection{Live cell experiments}

To determine the fraction of releasable vesicles at serial time points after tagging, I used two different approaches.

Investigating releasable vesicles during intrinsic network activity. In the first approach, releasable vesicles were tagged with the unconjugated 604.2 mouse monoclonal antibody against the lumenal domain of synaptotagmin 1 . The vesicles that were still releasing were then revealed by applying a secondary anti-mouse antibody conjugated to Cy5 (115-175146, Dianova, Hamburg, Germany) that had been dialyzed into Tyrode's solution (Table 5) prior to use to remove $\mathrm{NaN}_{3}$ and glycerine. The secondary antibody was applied for $1 \mathrm{~h}$ in the cell culture incubator at $37^{\circ} \mathrm{C}$ in a $5 \% \mathrm{CO}_{2}$ atmosphere at a dilution of $1: 100$ from an $\sim 0.4$ $\mathrm{mg} / \mathrm{ml}$ stock. The neurons were fixed immediately after the incubation with the secondary antibody, and a different secondary antibody conjugated to Cy3 was applied (115-165-146, Dianova, Hamburg, Germany). This revealed all tagged synaptic vesicles, since the first secondary antibody pulse was not saturating and did not diminish the fluorescence intensity that could be observed from the second secondary antibody. This allowed me to determine the fraction of initially tagged synaptic vesicles that was still releasable during intrinsic network activity of the neurons during the respective time points.

Investigating releasable vesicles during high-frequency stimulation. In the second approach, I used the same 604.2 mouse monoclonal antibody in an identical tagging protocol, but conjugated to CypHer5E, or a rabbit polyclonal antibody against the lumenal domain of VGAT, also conjugated to CypHer5E, in an identical tagging protocol. CypHer5E is brightly fluorescent in $\mathrm{pH} 5.5$, inside synaptic vesicles, and largely quenched in $\mathrm{pH} 7.3$, the extracellular medium. To determine the releasable fraction, neurons were then stimulated at 100 mA with a 385 Stimulus Isolator and an A310 Accupulser Stimulator (both from World Precision Instruments, Sarasota, FL, USA), using a custom-made platinum plate field stimulator with $8 \mathrm{~mm}$ distance between plates. To release the entire releasable population of synaptic vesicles, 600 action potentials were applied at $20 \mathrm{~Hz}$. To prevent the neurons from 
refilling recycled synaptic vesicles with protons, thereby lowering the $\mathrm{pH}$ inside recycled vesicles and increasing the fluorescence of CypHer5E before imaging could be completed, bafilomycin (Sigma-Aldrich, St. Louis, MO, USA) was applied at $0.5 \mu \mathrm{M}$. The neurons were imaged (1) before application of the stimulation and (2) after stimulation. After imaging, the neurons were fixed with methanol at $-20^{\circ} \mathrm{C}$ to preserve CypHer5E fluorescence and (3) flushed with a pH 5.5 buffer (Table 7), imaged, and then (4) flushed with a pH 7.4 buffer (Table 8), and imaged again. (4) gives the background signal to be subtracted from all other imaging conditions as CypHer5E in al vesicles is maximally quenched, (3) gives the total population of tagged vesicles at the imaging time point as CypHer5E in all vesicles is maximally fluorescent, (1) gives the internalized population of tagged vesicles as CypHer5e on the surface is maximally quenched while CypHer5e in internalized vesicles is maximally fluorescent, and (2) gives the non-releasable, inactive population of synaptic vesicles.

\section{Determining turnover dynamics of releasable synaptic vesicles with or without access} to newly produced synaptic vesicles. To determine the influx of new vesicles into the releasable population of vesicles, I first blocked all accessible epitopes with the unconjugated 604.2 mouse monoclonal antibody against the lumenal domain of synaptotagmin 1 by incubation with a 1:100 dilution from a $1 \mathrm{mg} / \mathrm{ml}$ stock for $2-3 \mathrm{~h}$ in the cell culture incubator. Then, I applied the same antibody, but conjugated to Atto $647 \mathrm{~N}$, for 30 min under the same conditions, $0 \mathrm{~h}, 12 \mathrm{~h}$, or $24 \mathrm{~h}$ after the initial blocking step. The increase of fluorescence represents the influx of new epitopes, which have not previously been blocked by the unconjugated monoclonal antibody. To disrupt the synthesis of new synaptic vesicles and check whether the releasable population could still be replenished, I applied $40 \mu \mathrm{M}$ anisomycin (Sigma-Aldrich, St. Louis, MO, USA), to block protein biosynthesis, or $10 \mu \mathrm{M}$ colchicine (Sigma-Aldrich, St. Louis, MO, USA), to disrupt the transport of precursor vesicles from the cell body to the synapse, starting during the blocking step.

Increasing synaptic activity. To check whether synaptic vesicle inactivation was dependent on vesicle age or vesicle usage, I repeated the first approach of vesicle functionality tracking outlined above, but under conditions where release frequency was increased for $12 \mathrm{~h}$ after the initial tagging. To increase release frequency, I added either $8 \mathrm{mM} \mathrm{CaCl}$ (Sigma-Aldrich, St. Louis, MO, USA), which decreases the activation threshold that triggers an action potential, or $20 \mu \mathrm{M}$ bicuculline (Sigma-Aldrich, St. Louis, MO, USA), which inhibits inhibitory GABAergic signalling in the culture network.

pHluorin imaging. Imaging of pHluorin responses to electrical stimulation (as described above) was performed in the presence of $10 \mu \mathrm{M}$ CNQX (Tocris Bioscience, Bristol, UK) and 
$50 \mu \mathrm{M}$ AP5 (Tocris Bioscience, Bristol, UK; Abcam, Cambridge, UK) to suppress intrinsic network activity.

Lysosome inhibition. To inhibit lysosomal degradation, in order to investigate differences in synaptic vesicle degradation after tagging with the 604.2 antibody conjugated to Atto $647 \mathrm{~N}$, the protease inhibitor leupeptin (Sigma-Aldrich, St. Louis, MO, USA) was applied at a concentration of $100 \mu \mathrm{M}$ for 1 day directly after tagging.

Table 2.7: pH 5.5 buffer for CypHer5E imaging.

\begin{tabular}{|l|l|l|}
\hline item & concentration & company \\
\hline $\mathrm{ddH}_{2} \mathrm{O}$ & base solvent & $\begin{array}{l}\text { from Lab Water Purification System from } \\
\text { Sartorius, Göttingen, Germany }\end{array}$ \\
\hline $\mathrm{MES}$ buffer & $20 \mathrm{mM}$ & Sigma-Aldrich, St. Louis, MO, USA \\
\hline $\mathrm{NaCl}$ & $150 \mathrm{mM}$ & Sigma-Aldrich, St. Louis, MO, USA \\
\hline Triton-X 100 & $0.1 \%(\mathrm{w} / \mathrm{v})$ & Sigma-Aldrich, St. Louis, MO, USA \\
\hline
\end{tabular}

Table 2.8: pH 7.4 buffer for CypHer5E imaging.

\begin{tabular}{|l|l|l|}
\hline item & concentration & company \\
\hline $\mathrm{ddH}_{2} \mathrm{O}$ & base solvent & $\begin{array}{l}\text { from Lab Water Purification System from } \\
\text { Sartorius, Göttingen, Germany }\end{array}$ \\
\hline $\mathrm{NaCl}$ & $137 \mathrm{mM}$ & Sigma-Aldrich, St. Louis, MO, USA \\
\hline $\mathrm{KCl}$ & $2.7 \mathrm{mM}$ & Sigma-Aldrich, St. Louis, MO, USA \\
\hline $\mathrm{Na}_{2} \mathrm{KPO}_{4}$ & $10 \mathrm{mM}$ & Sigma-Aldrich, St. Louis, MO, USA \\
\hline $\mathrm{KH}_{2} \mathrm{PO}_{4}$ & $2 \mathrm{mM}$ & Sigma-Aldrich, St. Louis, MO, USA \\
\hline Triton-X 100 & $0.1 \%(w / v)$ & Sigma-Aldrich, St. Louis, MO, USA \\
\hline
\end{tabular}

\subsection{Fixation, permeabilization, and immunostaining}

Fixation was usually performed with 4\% PFA (Sigma-Aldrich, St. Louis, MO, USA) for 10 min on ice followed by $30 \mathrm{~min}$ on room temperature. Alternatively, fixation with methanol (SigmaAldrich, St. Louis, MO, USA) was performed for $20 \mathrm{~min}$ at $-20^{\circ} \mathrm{C}$ after live cell experiments where CypHer5E-conjugated antibodies were used, since CypHer5E loses its fluorescence after PFA fixation. Following PFA fixation, cells were washed with PBS 2x for 5 min, followed by quenching of unreacted PFA with $100 \mathrm{mM} \mathrm{NH}_{4} \mathrm{Cl}$ in PBS for 20 min. Cells were then permeabilized in staining solution (Table 9) $3 x$ for $5 \mathrm{~min}$, followed by incubation with the primary antibody (see Table 13) in staining solution for $1 \mathrm{~h}$ by flipping the cover slip with cultured neurons upside down onto an $80 \mu \mathrm{l}$ droplet of staining solution with antibody on parafilm (Bemis, Neenah, WI, USA) in a humidified chamber. After the primary antibody incubation, cells were washed $3 x$ for 5 min with staining solution, followed by incubation with the secondary antibody (see Table 14 ) in staining solution for $1 \mathrm{~h}$, as described for the 
primary antibody. Subsequently, cells were subjected to stringency washes: $3 x$ for 5 min with blocking solution (Table 10), followed by $3 x$ for 5 min in high-salt PBS (Table 11), followed by $2 x$ for 5 min with PBS. The cells were then embedded in $10 \mu$ of Mowiol (Table 12) on a cover glass (Thermo Fisher Scientific, Waltham, MA, USA) and dried overnight at room temperature or embedded in melamine for thin-sectioning (see below). The samples were stored on $4^{\circ} \mathrm{C}$ until imaging.

Table 2.9: Staining solution for post-fixation immunostainings.

\begin{tabular}{|l|l|l|}
\hline item & concentration & company \\
\hline PBS & base solution & see Table 6 \\
\hline BSA & $2.5 \%(w / v)$ & Applichem, Darmstadt, Germany \\
\hline Triton-X 100 & $0.1 \%(w / v)$ & Sigma-Aldrich, St. Louis, MO, USA \\
\hline
\end{tabular}

Table 2.10: Blocking solution for post-fixation immunostainings.

\begin{tabular}{|l|l|l|}
\hline item & concentration & company \\
\hline PBS & base solution & see Table 6 \\
\hline BSA & $2.5 \%(w / v)$ & Applichem, Darmstadt, Germany \\
\hline
\end{tabular}

Table 2.11: High-salt PBS.

\begin{tabular}{|l|l|l|}
\hline item & concentration & company \\
\hline $\mathrm{ddH}_{2} \mathrm{O}$ & base solvent & $\begin{array}{l}\text { from Lab Water Purification System from } \\
\text { Sartorius, Göttingen, Germany }\end{array}$ \\
\hline $\mathrm{NaCl}$ & $500 \mathrm{mM}$ & Sigma-Aldrich, St. Louis, MO, USA \\
\hline $\mathrm{KCl}$ & $2.7 \mathrm{mM}$ & Sigma-Aldrich, St. Louis, MO, USA \\
\hline $\mathrm{Na}_{2} \mathrm{KPO}_{4}$ & $10 \mathrm{mM}$ & Sigma-Aldrich, St. Louis, MO, USA \\
\hline $\mathrm{KH}_{2} \mathrm{PO}_{4}$ & $2 \mathrm{mM}$ & Sigma-Aldrich, St. Louis, MO, USA \\
\hline
\end{tabular}

Table 2.12: Mowiol solution (prepared by heating to $50-60^{\circ} \mathrm{C}$ ).

\begin{tabular}{|l|l|l|}
\hline item & concentration & company \\
\hline $\mathrm{ddH}_{2} \mathrm{O}$ & $68 \%(\mathrm{w} / \mathrm{w})$ & $\begin{array}{l}\text { from Lab Water Purification System from } \\
\text { Sartorius, Göttingen, Germany }\end{array}$ \\
\hline glycerol & $23 \%(\mathrm{w} / \mathrm{w})$ & Sigma-Aldrich, St. Louis, MO, USA \\
\hline Mowiol 4-88 & $9 \%(\mathrm{w} / \mathrm{w})$ & Merck KGaA, Darmstadt, Germany \\
\hline TRIS $(\mathrm{pH} 8.5)$ & $100 \mathrm{mM}$ & Sigma-Aldrich, St. Louis, MO, USA \\
\hline
\end{tabular}


Table 2.13: List of primary antibodies used for post-fixation immunostainings (all diluted from a 1 $\mathrm{mg} / \mathrm{ml}$ stock). The antibodies were from Synaptic Systems, Göttingen, Germany or Cell Signalling Technology, Cambridge, UK.

\begin{tabular}{|l|l|l|l|l|}
\hline antibody against & species & dilution & catalogue \# & company \\
\hline PSD95 & rabbit & $1: 100$ & $3450 S$ & Cell Signalling \\
\hline Rab7 & rabbit & $1: 100$ & 9367 & Cell Signalling \\
\hline SNAP25 & rabbit & $1: 100$ & 111002 & Synaptic Systems \\
\hline synapsin I/II & rabbit & $1: 500$ & 106002 & Synaptic Systems \\
\hline synaptophysin & guiena pig & $1: 500$ & 101004 & Synaptic Systems \\
\hline synaptophysin & rabbit & $1: 100$ & 101011 & Synaptic Systems \\
\hline synaptotagmin 1 & mouse & $1: 100$ & 105311 & Synaptic Systems \\
\hline synaptotagmin 1 & mouse & $1: 100$ & 105311 AT1 & Synaptic Systems \\
\hline synaptotagmin 1 & rabbit & $1: 500$ & 105102 & Synaptic Systems \\
\hline syntaxin 1 & rabbit & $1: 100$ & 110302 & Synaptic Systems \\
\hline syntaxin 16 & rabbit & $1: 100$ & 110162 & Synaptic Systems \\
\hline VATPase & rabbit & $1: 100$ & 109002 & Synaptic Systems \\
\hline VAMP2 & rabbit & $1: 500$ & 104202 & Synaptic Systems \\
\hline VAMP4 & rabbit & $1: 100$ & 136002 & Synaptic Systems \\
\hline VGlut 1/2 & rabbit & $1: 100$ & 135503 & Synaptic Systems \\
\hline
\end{tabular}

Table 2.14: List of secondary antibodies used for post-fixation immunostainings (all diluted from an $\sim 0.4 \mathrm{mg} / \mathrm{ml}$ stock). The antibodies were from Dianova, Hamburg, Germany or Active Motif, Carlsbad, CA, USA.

\begin{tabular}{|l|l|l|l|l|l|}
\hline antibody against & fluorophore & species & dilution & catalogue \# & company \\
\hline guinea pig IgG & AMCA & donkey & $1: 100$ & $706-155-148$ & Dianova \\
\hline guinea pig IgG & Alexa 488 & donkey & $1: 100$ & $706-545-148$ & Dianova \\
\hline guinea pig IgG & Cy3 & donkey & $1: 100$ & $706-165-148$ & Dianova \\
\hline mouse IgG & Cy3 & goat & $1: 100$ & $115-165-146$ & Dianova \\
\hline mouse IgG & Cy5 & goat & $1: 100$ & $115-175-146$ & Dianova \\
\hline rabbit IgG & Cy3 & goat & $1: 100$ & $111-165-144$ & Dianova \\
\hline rabbit IgG & Chromeo 494 & goat & $1: 50$ & 15042 & Active Motif \\
\hline
\end{tabular}

\subsection{Metabolic labelling with AHA or ${ }^{15} \mathrm{~N}$ leucine}

For labelling of newly produced proteins with L-azidohomoalanine (AHA; Thermo Fisher Scientific, Waltham, MA, USA), neurons were placed in a methionine-free labelling medium (Table 15) for $9 \mathrm{~h}$, washed with Tyrode's solution (Table 4), and placed back into their original culture medium until further processing by live tagging of releasable synaptic vesicles or fixation and immunostaining (see above). For CLICK chemistry conjugation of AHA to fluorophores, the commercial Click-IT Cell Reaction Buffer Kit (Invitrogen, Waltham, MA, 
USA) was used as per the manufacturer's instructions with $5 \mathrm{mM}$ Chromeo 494-alkyne (Jena Bioscience, Jena, Germany) after fixation and before immunostaining.

For labelling of newly produced proteins for nanoSIMS imaging, $2.4 \mathrm{mM}{ }^{15} \mathrm{~N}$ leucine (SigmaAldrich, St. Louis, MO, USA) was added to the neuronal culture medium (see Table 4), which contains $0.8 \mathrm{mM}$ leucine. The neurons were incubated with ${ }^{15} \mathrm{~N}$ leucine for $1-3$ days before antibody tagging of releasable synaptic vesicles (see above).

Table 2.15: Methionine-free medium for labelling of newly produced proteins with AHA.

\begin{tabular}{|l|l|l|}
\hline item & concentration & company \\
\hline $\begin{array}{l}\text { DMEM w/o Met, } \\
\text { Gln, Cys, pyruvate }\end{array}$ & base solvent & Thermo Fisher Scientific, Waltham, MA, USA \\
\hline AHA & $50 \mu \mathrm{M}$ & Thermo Fisher Scientific, Waltham, MA, USA \\
\hline $\mathrm{MgCl}_{2}$ & $812 \mu \mathrm{M}$ & Sigma-Aldrich, St. Louis, MO, USA \\
\hline HEPES & $6.5 \mathrm{mM}$ & Merck KGaA, Darmstadt, Germany \\
\hline L-cysteine & $260 \mu \mathrm{M}$ & Thermo Fisher Scientific, Waltham, MA, USA \\
\hline B27 supplement & $1: 50$ & Thermo Fisher Scientific, Waltham, MA, USA \\
\hline GlutaMAX & $1: 100$ & Thermo Fisher Scientific, Waltham, MA, USA \\
\hline
\end{tabular}

\subsection{SNAP-tag labelling and TEV protease cleavage in living neurons}

To conjugate SNAP-tags in living cells to fluorophores, the cell permeable dyes TMR-Star and 647-SiR (both from New England Biolabs, Ipswich, MA, USA) were used in a concentration of $1 \mu \mathrm{M}$ each. TMR-Star was usually applied first (although the sequence is interchangeable), for $15-30 \mathrm{~min}$ at $37^{\circ} \mathrm{C}$ in a $5 \% \mathrm{CO}_{2}$ atmosphere in a cell culture incubator. The neurons were then allowed to rest for 1 day, before the second dye was applied for 24 hours. The extended second labelling step was necessary to achieve detectable fluorescence signal, presumably because there was less protein available for the second labelling step. All labelling steps were carried out in the neurons' own culture medium and the first labelling step was performed 3-4 days after transfection (see below).

\subsection{Embedding and thin-sectioning}

STED microscopy requires melamine embedding, while nanoSIMS requires LR White embedding.

Melamine embedding for STED microscopy. Samples to be imaged in super-resolution microscopy had to be embedded in melamine resin for ultrathin-sectioning to achieve an increased z-resolution that could match the xy-resolution provided by the STED microscope. After immunostaining, a BEEM capsule (BEEM Inc., West Chester, PA, USA) with its bottom 
cut off was placed on the coverslip of the cultured neurons and melamine solution (Table 16) was added into the opening of the capsule until the cell layer was covered completely. The melamine was then allowed to penetrate completely into the cells overnight at room temperature by placing the sample into a box containing silica beads (Sigma-Aldrich, St. Louis, MO, USA). The sample was then dried for $24 \mathrm{~h}$ at $40^{\circ} \mathrm{C}$ in the box with silica beads. Afterwards, the BEEM capsule was filled up with EPON resin prepared with a commercially available kit (Struers, Willich, Germany) and incubated at $60^{\circ} \mathrm{C}$ for $48 \mathrm{~h}$ to harden. After removing the BEEM capsule and the cover slip, the melamine around the region of interest was then trimmed with a razor blade and the melamine blocks were cut into $50 \mathrm{~nm}$ thin sections with an EM UC6 ultramicrorome (Leica, Wetzlar, Germany). The sections were collected floating on water and dried on a coverslip before embedding in Mowiol (Table 12) for imaging.

LR White embedding for nanoSIMS. For nanoSIMS imaging, the samples were embedded in LR White to avoid the high background signal from the amines in melamine. For that, the samples were dehydrated after immunostaining through 5 min washing with 30\% ethanol (Sigma-Aldrich, St. Louis, MO, USA) in $\mathrm{dd}_{2} \mathrm{O}$, followed by $3 \times 5$ min washes with $50 \%$ ethanol. The samples were then incubated in LR White medium grade acrylic resin (London Resin Company, London, UK) in 50\% ethanol (1:1 v/v mixture) for $30 \mathrm{~min}$, followed by $60 \mathrm{~min}$ incubation in pure LR White, both rotating at $50 \mathrm{rpm}$. For the final embedding step, samples were then incubated with pure LR White plus LR White accelerator (London Resin Company, London, UK) diluted into it 1:20 for $15 \mathrm{~min}$ on room temperature followed by $90 \mathrm{~min}$ on $40^{\circ} \mathrm{C}$. The samples were then cut into $200 \mathrm{~nm}$ thin sections as described above for melamine embedding. The thicker sections were necessary to provide sufficient volume for preionization implantation during nanoSIMS imaging. The sections were collected floating on water and placed on a silicon wafer (Siegert Wafer $\mathrm{GmbH}$, Aachen, Germany) for imaging.

Table 2.16: Melamine solution (mixed by agitation at $250 \mathrm{rpm}$ on a horizontal shaker until melamine was completely dissolved).

\begin{tabular}{|l|l|l|}
\hline item & concentration & company \\
\hline melamine & $68.3 \%(\mathrm{w} / \mathrm{w})$ & TCl, Chiyoda, Tokyo, Japan \\
\hline $\mathrm{ddH}_{2} \mathrm{O}$ & $29.3 \%(\mathrm{w} / \mathrm{w})$ & $\begin{array}{l}\text { from Lab Water Purification System from } \\
\text { Sartorius, Göttingen, Germany }\end{array}$ \\
\hline $\begin{array}{l}\text { p-tuloenesulfonic } \\
\text { acid monohydrate }\end{array}$ & $2.4 \%(\mathrm{w} / \mathrm{w})$ & Sigma-Aldrich, St. Louis, MO, USA \\
\hline
\end{tabular}




\subsection{Cloning}

GCaMP6, sypHy, and mOrange2 were obtained from Addgene (Cambridge, MA, USA; plasmid numbers 40753, 24478, and 54650 respectively). The superecliptic GFP from the original sypHy construct was cut out by Agel restriction and replaced with the sequence for the also $\mathrm{pH}$-sensitive mOrange2 fluorescent protein. The mOrange2 sequence was PCRamplified (Table 17, Table 18) with added Agel restriction sites from the Addgene vector. Restriction was carried out for $1 \mathrm{~h}$ each at $37^{\circ} \mathrm{C}$ (Table 19) and the DNA was purified with a commercial PCR cleaning kit as per the manufacturer's instructions (Qiagen, Hilden, Germany). Ligation was carried out (Table 20) and the resulting plasmid was then transformed into competent bacteria by incubating $50 \mu \mathrm{l}$ of bacteria (Bioline, London, UK) with $1 \mu \mathrm{l}$ of ligation mix for $20 \mathrm{~min}$ on ice, followed by a heat shock of $42^{\circ} \mathrm{C}$ for $42 \mathrm{~s}$, followed by 2 min on ice, followed by adding $500 \mu$ of LB medium (Sigma-Aldrich, St. Louis, MO, USA) and incubating for $1 \mathrm{~h}$ at $37^{\circ} \mathrm{C}$ before finally plating the transformed bacteria on agar plates containing the appropriate antibiotic. Clones were picked after overnight incubation at $37^{\circ} \mathrm{C}$ and midis were produced with a commercial kit as per the manufacturer's instructions (Macherey-Nagel, Düren, Germany). Correct orientation of the insert was confirmed by sequencing (Seqlab, Göttingen, Germany). YFP-SNAP25 was a kind gift from Reinhard Jahn (Göttingen, Germany) and was constructed by cloning SNAP25 into the pEYFP-C1 vector (Clontech, Otsu, Japan) via the Sacl and Apal restriction sites in a way similar to the procedure described above. Wild-type CSPa and mutated CSPa in which the cysteines of the cysteine string critical for palmitoylation and thus insertion into the vesicle membrane have been replaced with serines (Sharma et al., 2012) were obtained via custom synthesis through a commercial vendor (Invitrogen, Waltham, MA, USA).

Table 2.17: Reaction mix for PCR amplification of DNA.

\begin{tabular}{|l|l|l|}
\hline item & volume & company \\
\hline vector & $1 \mu \mathrm{l}($ of $0.25 \mu \mathrm{g} / \mu \mathrm{l})$ & $\begin{array}{l}\text { Addgene, Cambridge, MA, USA or own } \\
\text { production or kind gifts from other labs }\end{array}$ \\
\hline forward primer & $5 \mu \mathrm{l}($ of $50 \mu \mathrm{M})$ & Sigma-Aldrich, St. Louis, MO, USA \\
\hline reverse primer & $5 \mu \mathrm{l}($ of $50 \mu \mathrm{M})$ & Sigma-Aldrich, St. Louis, MO, USA \\
\hline $5 x$ reaction buffer & $10 \mu \mathrm{l}$ & New England Biolabs, Ipswich, MA, USA \\
\hline dNTPs & $1 \mu \mathrm{l}$ & Thermo Fisher Scientific, Waltham, MA, USA \\
\hline $\begin{array}{l}\text { Phusion } \\
\text { polymerase }\end{array}$ & $0.5 \mu \mathrm{l}$ & New England Biolabs, Ipswich, MA, USA \\
\hline dd $\mathrm{H}_{2} \mathrm{O}$ & to $50 \mu \mathrm{l}$ & $\begin{array}{l}\text { from Lab Water Purification System from } \\
\text { Sartorius, Göttingen, Germany }\end{array}$ \\
\hline
\end{tabular}


Table 2.18: PCR programme for DNA amplification, steps 2)-4) repeated 30x.

\begin{tabular}{|l|l|l|l|}
\hline step & rationale & temperature & time \\
\hline 1$)$ & initial denaturation & $98^{\circ} \mathrm{C}$ & $1 \mathrm{~min}$ \\
\hline 2$)$ & denaturation & $98^{\circ} \mathrm{C}$ & $10 \mathrm{~s}$ \\
\hline 3$)$ & annealing & variable, depending on primer & $15 \mathrm{~s}$ \\
\hline 4$)$ & extension & $72^{\circ} \mathrm{C}$ & $15 \mathrm{~s}$ per kb \\
\hline 5$)$ & final extension & $72^{\circ} \mathrm{C}$ & 5 min \\
\hline 6$)$ & storing & $4^{\circ} \mathrm{C}$ & until stopped \\
\hline
\end{tabular}

Table 2.19: Reaction mix for DNA restriction digest.

\begin{tabular}{|l|l|l|}
\hline item & volume & company \\
\hline $\begin{array}{l}\text { PCR product } \\
\text { or } \\
\text { vector }\end{array}$ & $\begin{array}{l}40 \mu \mathrm{l} \\
\text { or } \\
1 \mu \mathrm{l}(\text { of } \sim 0.5 \mu \mathrm{g} / \mu \mathrm{l})\end{array}$ & $\begin{array}{l}\text { Addgene, Cambridge, MA, USA or own } \\
\text { production or kind gifts from other labs }\end{array}$ \\
\hline enzyme 1 & $2 \mu \mathrm{l}$ & Thermo Fisher Scientific, Waltham, MA, USA \\
\hline enzyme 2 & $2 \mu \mathrm{l}$ & Thermo Fisher Scientific, Waltham, MA, USA \\
\hline $10 x$ buffer & $5 \mu \mathrm{l}$ & Thermo Fisher Scientific, Waltham, MA, USA \\
\hline $\mathrm{ddH}_{2} \mathrm{O}$ & to $50 \mu \mathrm{l}$ & $\begin{array}{l}\text { from Lab Water Purification System from } \\
\text { Sartorius, Göttingen, Germany }\end{array}$ \\
\hline
\end{tabular}

Table 2.20: Reaction mix for DNA ligation.

\begin{tabular}{|l|l|l|}
\hline item & volume & company \\
\hline T4 ligase & $1 \mu \mathrm{l}$ & Thermo Fisher Scientific, Waltham, MA, USA \\
\hline 10x reaction buffer & $2 \mu \mathrm{l}$ & Thermo Fisher Scientific, Waltham, MA, USA \\
\hline vector & $1 \mu \mathrm{l}$ & $\begin{array}{l}\text { Addgene, Cambridge, MA, USA or own } \\
\text { production or kind gifts from other labs }\end{array}$ \\
\hline PCR product & $3 \mu \mathrm{l}$ or $7 \mu \mathrm{l}$ & see Table 15 and Table 16 \\
\hline $\mathrm{ddH}_{2} \mathrm{O}$ & to $20 \mu \mathrm{l}$ & $\begin{array}{l}\text { from Lab Water Purification System from } \\
\text { Sartorius, Göttingen, Germany }\end{array}$ \\
\hline
\end{tabular}

\subsection{Transfections}

Neurons were transfected with a commercially available calcium phosphate transfection kit (Promega, Fitchburg, WI, USA), using a protocol adapted from the manufacturer's instructions. To minimize excitotoxicity caused by excessive release of neurotransmitter due to the osmolarity shock associated with buffer changes, neurons were pre-treated for 15-30 min at $37^{\circ} \mathrm{C}$ in a $5 \% \mathrm{CO}_{2}$ atmosphere in a cell culture incubator in fDMEM (Table 21). $50 \mu \mathrm{l}$ of transfection mix (Table 22) per $1 \mathrm{ml}$ of fDMEM per coverslip was then prepared and added after 15 min incubation at room temperature for another 15 min incubation with the neurons in the incubator. The neurons were then washed 3x on/off with fDMEM to remove excess calcium phosphate crystals and placed back into their original culture medium. Transfection was usually allowed for 3-4 days before further experiments. 
Table 2.21: Fresh DMEM (fDMEM) for transfection of neurons (adjusted to $\mathrm{pH} 7.5$ before use).

\begin{tabular}{|l|l|l|}
\hline item & concentration & company \\
\hline DMEM & base solvent & Thermo Fisher Scientific, Waltham, MA, USA \\
\hline $\mathrm{MgCl}_{2}$ & $10 \mathrm{mM}$ & Sigma-Aldrich, St. Louis, MO, USA \\
\hline HEPES & $5 \mathrm{mM}$ & Merck KGaA, Darmstadt, Germany \\
\hline
\end{tabular}

Table 2.22: Transfection mix for one 12-well plate of rat hippocampal neurons (HBS added last, followed by thorough vortexing for $\sim 30 \mathrm{~s}$ ).

\begin{tabular}{|l|l|l|}
\hline item & amount & company \\
\hline $\mathrm{CaCl}_{2}$ & $80 \mu \mathrm{l}$ & Promega, Fitchburg, WI, USA \\
\hline DNA & $2 \mu \mathrm{g}$ per coverslip & own cloning \\
\hline $\mathrm{ddH}_{2} \mathrm{O}$ & to $300 \mu \mathrm{l}$ & Promega, Fitchburg, WI, USA \\
\hline $\mathrm{HBS}$ & $300 \mu \mathrm{l}$ & Promega, Fitchburg, WI, USA \\
\hline
\end{tabular}

\subsection{Imaging}

Imaging on different microscopes was performed as required by the experiment. Which technique was used follows from the description of the experiments in the Figure Legends.

STED. For STED super-resolution imaging, including two-colour STED microscopy, a TCS SP5 STED microscope (Leica, Wetzlar, Germany) equipped with an HCX plan apochromat 100x 1.4 NA oil-immersion STED objective, and operated via the LAS AF imaging software (Leica, Wetzlar, Germany), was used. Atto 647N and Chromeo 494 were excited with pulsed diode lasers (PicoQuant, Berlin, Germany) at $640 \mathrm{~nm}$ and $531 \mathrm{~nm}$, respectively. The STED depletion beam was generated by a MaiTai Ti:Sapphire laser (Spectra Physics, Mountain View, CA, USA) tuned to $750 \mathrm{~nm}$. For acquiring confocal images, the same microscope was used, either with the objective named above, or with an HCX plan apochromat $63 \times 1.4 \mathrm{NA}$ oil-immersion objective.

Live imaging. Live imaging was performed on a Nikon Ti-E epifluorescence microscope (Nikon Corporation, Chiyoda, Tokyo, Japan) equipped with a plan apochromat 60x 1.4 NA oil-immersion objective and a plan apochromat 100x 1.4 NA oil-immersion objective, an HBO-100W lamp, an IXON X3897 Andor camera (Andor, Belfast, Northern Ireland, UK) and an OKOLab cage incubator system (OKOLab, Ottaviano, Italy), and operated with the NISElements AR software (Nikon Corporation, Chiyoda, Tokyo, Japan). The same microscope was also occasionally used for acquiring images of fixed samples. Alternatively, an inverted epifluorescence Olympus microscope equipped with a 60x 1.35 NA oil-immersion objective, a 100W mercury lamp and a charge-coupled device camera (all devices from Olympus 
Corporation, Shinjuku, Tokyo, Japan), and operated via the cell^P software (Olympus Corporation, Shinjuku, Tokyo, Japan), was used.

nanoSIMS. The nanoSIMS imaging was performed on a Cameca nanoSIMS 50L instrument (Cameca, Gennevilliers, France). The scanning parameters were $512 \times 512$ pixels with a pixel size of $35 \mathrm{~nm}$ and a dwell time of $4000 \mu$ s per pixel.

\subsection{Data Analysis}

Data analysis was performed with custom-written MATLAB (MathWorks, Natick, MA, USA) routines, ImageJ (Wayne Rasband, NIH, Bethesda, MD, USA), and SigmaPlot (Systat Software Inc., Erkrath, Germany). To correct for synapse size, the fluorescence level of synaptotagmin 1 live tagging in releasable synaptic vesicles was corrected for the fluorescence level of synaptophysin whenever neurons were processed for coimmunostainigs. For display purposes only, images were deconvolved with the Huygens Essentials software (Scientific Volume Imaging, Hilversum, Netherlands), based on built-in algorithms that were adjusted for the specific imaging parameters.

\subsection{Statistical Analysis}

Student's t-test or one-way ANOVA with Tukey's post-hoc test were used to calculate statistical significance, as appropriate. A p-value of $<0.05$ was considered to be statistically significant.

\subsection{Calculation of SNAP25 copy numbers on young and old synaptic vesicles}

To calculate the copy number of SNAP25 on old, inactive and young, releasable synaptic vesicles from the ratio of these (determined in Figure 3.11), it is only necessary to know the copy number of SNAP25 on the average synaptic vesicle and the fraction of old, inactive and young, releasable synaptic vesicles. These parameters relate to each other in the following manner:

$$
N_{\text {mean }}=N_{\text {young }} * F_{\text {young }}+N_{\text {old }} * F_{\text {old }}
$$

with

- $\quad N_{\text {mean }}$ is the average copy number of SNAP25 per synaptic vesicle (average of both young and old vesicles), which is 1.8 (Takamori et al., 2006).

- $\quad N_{\text {young }}$ is the copy number of SNAP25 on young, releasable synaptic vesicles. 
- $F_{\text {young }}$ is the fraction of young, releasable synaptic vesicles in the population of vesicles from which SNAP25 was quantified, which was rat cortices. This is $\sim 0.136$ (Marra et al., 2012).

- $\quad N_{\text {old }}$ is the copy number of SNAP25 on old, inactive synaptic vesicles.

- $F_{\text {old }}$ is the fraction of old, inactive synaptic vesicles in the population of vesicles from which SNAP25 was quantified, which was rat cortices. This is $\sim 0.864$ (Marra et al., 2012).

As the ratio of the copy number of SNAP25 on old over young synaptic vesicles was determined experimentally in this Thesis (Figure 3.11), one can substitute and rearrange the formula to arrive at the copy number of SNAP25 on young synaptic vesicles:

$$
N_{\text {young }}=\frac{F_{\text {young }}+r_{\text {old } / \text { young }} * F_{\text {old }}}{N_{\text {mean }}}
$$

with

- $r_{\text {old } / \text { young }}$ is the ratio of copies of SNAP25 on old over young synaptic vesicles, which is $\sim 1.87$ (Figure 3.11).

- The other parameters are defined in Equation (3).

This yields an average copy number of 0.97 SNAP25 proteins per young synaptic vesicle. From this, the copy number of SNAP25 on old synaptic vesicles is easily calculated from Equation (3) to be 1.94, on average.

\subsection{Prediction of protein damage from protein lifetimes}

To predict the protein damage synaptic vesicles accumulate during their lifetime and match this with the time points of functional changes during a vesicle's lifetime, I used data from a published study on synaptic protein lifetimes in rat hippocampal cultures (Cohen et al., 2013). I extracted the lifetimes of all synaptic vesicle proteins that had been measured in this study and calculated the average lifetime of the entire vesicle by weighting these lifetimes by protein copy number (Wilhelm et al., 2014; see Figure 3.17A). The average lifetime of a synaptic vesicle was thus determined to be $3.8 \pm 0.8$ (mean \pm SD) days. I then assumed that degradation would usually occur only after damage to the proteins in question and thus equated lifetimes with damage accumulation. Assuming a Gaussian distribution of degradation and damage accumulation, with the peak at 3.8 days and a standard deviation of 0.8 days (see above), I could thus plot a probability density function for damage accumulation on the average synaptic vesicle (Figure 3.17B). On the same graph, I plotted 
the approximate time point at which the synaptic vesicle enters the releasable population after biogenesis and the approximate time point at which it leaves the releasable population (time constant $\mathrm{T}=0.5$ days, derived from Figure 3.5C, times 2 for correcting for the fact that tagging occurs for vesicles after half their lifetime in the releasable population has already passed, on average (see Chapter 2.13), plus $12 \mathrm{~h}$ for transport to the synapse). Finally, I plotted the average time point at which the vesicle leaves the synapse for degradation (time constant $\mathrm{T}=1.6$ days, derived from Figure $3.4 \mathrm{~A}, \mathrm{~B}$, times 2 for correcting for the fact that tagging occurs for vesicles after half their lifetime in the releasable population has already passed, on average (see Chapter 2.13), plus $12 \mathrm{~h}$ for transport to the synapse), which intersects with the probability density plot at $\sim 50 \%$ damage, which equates to the average lifetime of the synaptic vesicle of 3.8 days. 


\section{Results}

\subsection{Live-cell tagging with antibodies allows investigation of the synaptic vesicle life cycle}

To follow the life cycle of synaptic vesicles in hippocampal cultures, I made use of antibodies against the vesicle lumenal domains of synaptic vesicle proteins (Figure 3.1A). The only two proteins for which reliable antibodies against their lumenal domain are available are synaptotagmin 1 (Kraszewski et al., 1995; Matteoli et al., 1992) and VGAT (Martens et al., 2008). Synaptotagmin 1 is the main calcium sensor of synaptic vesicle release (Geppert et al., 1994) and VGAT is the GABA transporter of synaptic vesicles in inhibitory synapses (McIntire et al., 1997). Since the majority of synapses in our culture system are excitatory glutamatergic synapses (only $\sim 5-10 \%$ are GABAergic; Benson et al., 1994) and since the synaptotagmin 1 antibody is better characterized and offers more experimental flexibility due to its monoclonal nature, the experiments presented in this Thesis are almost exclusively based on the synaptotagmin 1 antibody. The antibody localizes well to synaptic compartments after live-tagging, as demonstrated by co-immunostaining for the synaptic vesicle marker synaptophysin (Figure 3.1B), which localizes to synaptic vesicles better than any other protein (Rizzoli et al., 2006; Takamori et al., 2006). The co-immunostaining for synaptophysin is regularly used throughout this Thesis to correct for synapse size. Once the antibodies are bound to the lumenal domain of synaptic vesicle proteins during release and recycling of synaptic vesicles (Figure 3.1A), they stay bound for up to 10 days (Figure $3.1 C, D$ ). The entire recycling pool of $\sim 25 \%$ of internalized synaptic vesicles (Marra et al., 2012; Rose et al., 2013) can be labelled by application of the antibody for $1 \mathrm{~h}$ (Figure 3.2). No external stimulation is needed, due to the intrinsic network activity of the cultures (see Figure 3.10 in Chapter 3.4). The antibodies recapitulate the entire life cycle of the synaptic vesicles from the point of tagging up until degradation in the cell body (Figure 3.3). The use of antibodies means minimal disruption of physiological processes, as the tagged vesicles remain fully functional in terms of release in response to stimulation (see Figure 3.4 in Chapter 3.2) and no overexpression of proteins is required, which might potentially alter protein levels. Taken together, this approach allows undisturbed functional observations of synaptic vesicles throughout the majority of their life cycle. 
A

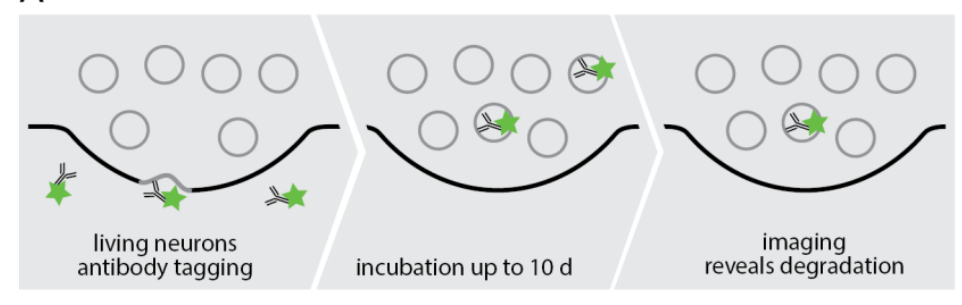

B

synaptophysin synaptotagmin 1 overlay permeabilised live-tagging
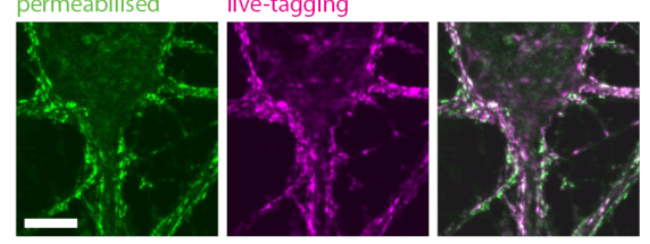

$\mathrm{D}$

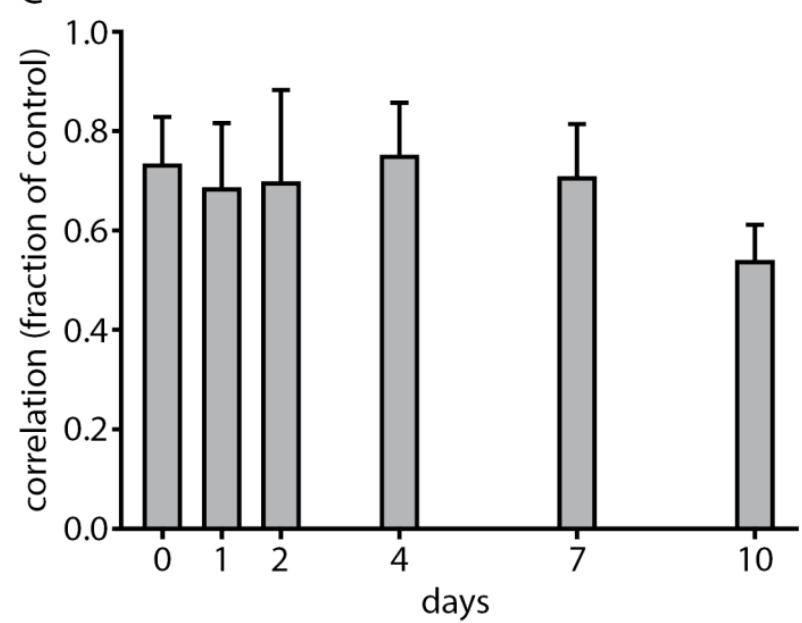

$\mathrm{pH} 5.5+$ peptide (100x molar excess) $\square \mathrm{pH} 7.4$ + peptide (100x molar excess)

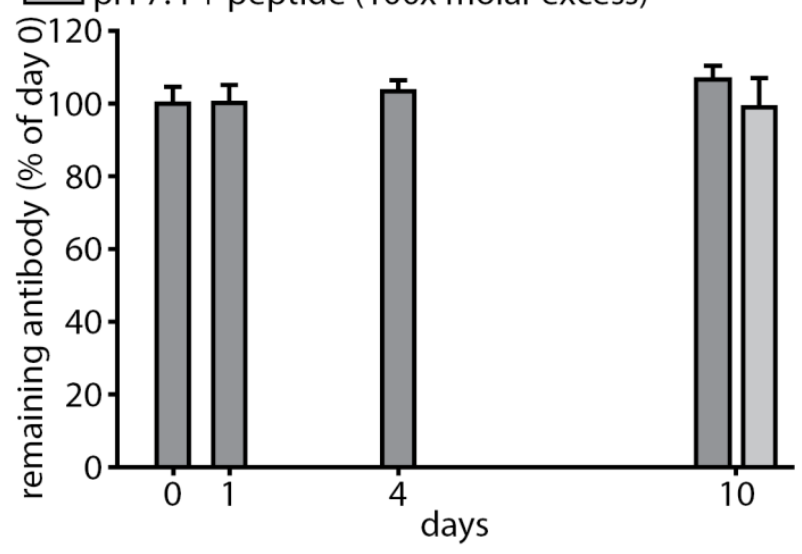

Figure 3.1: Synaptic vesicles can be reliably tagged with antibodies and followed for up to 10 day in living hippocampal cultures.

(A) The principle of antibody tagging: the antibody is added to the culture medium, where it binds to the lumenal domain of its target protein on releasing synaptic vesicles (left panel), followed by uptake of the antibody together with the vesicle (middle panel), which move together until degradation of vesicle and antibody or imaging of the directly fluorescently conjugated antibodies (right panel).

(B) A co-immunostaining with synaptophysin antibodies of synapses live-tagged with synaptotagmin 1 antibodies shows a high degree of signal overlap, indicating that the live-tagging antibody localizes mostly to synaptic compartments.

(C) A Pearson's correlation coefficient analysis of fluorescence signal from live-tagging synaptotagmin 1 antibodies with that from synaptophysin antibodies reveals a high degree of co-localization for up to 10 days $(n=3,3,2,3,3,2$ independent experiments per respective time point, at least 10 neurons sampled per experiment). The correlation is expressed as fraction of the Pearson's correlation coefficient of the synaptotagmin 1 live-tagging and a co-immunostaining for synaptophysin and the Pearson's correlation coefficient of two different synaptophysin antibodies (control).

(D) The live-tagging antibody for the lumenal domain of synaptotagmin 1 stays bound for up to 10 days, as revealed by staining fixed and permeabilized neurons with them and storing the neurons for up to 10 days in the presence of a molar excess of the antigenic peptide that was used to raise the antibody ( $n=3$ independent experiments per time point, at least 10 neurons sampled per experiment). All data represent the mean \pm SEM. 


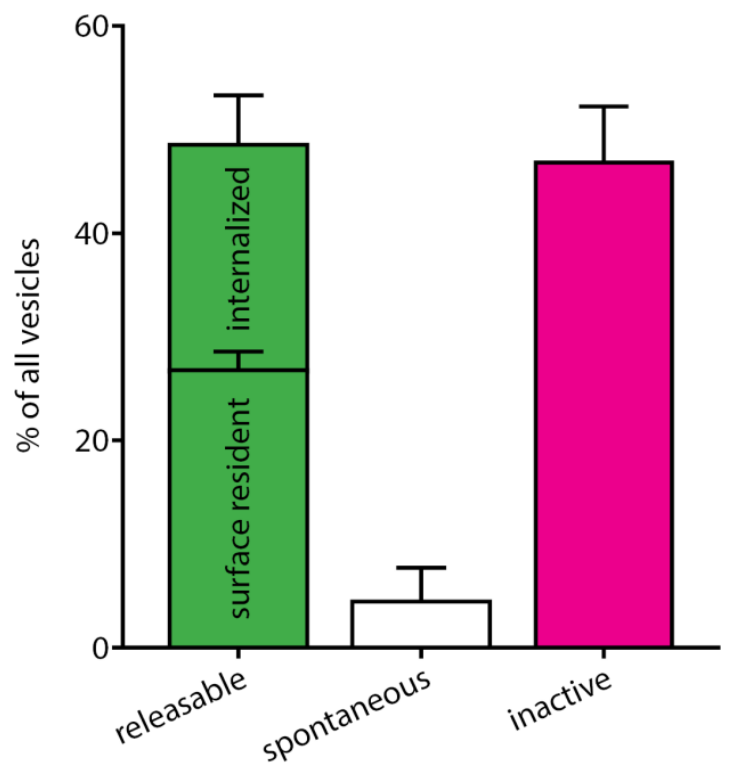

Figure 3.2: Live-tagging of synaptotagmin 1 in culture labels the complete releasable population of synaptic vesicles.

This releasable population consists of vesicles that are capable of responding to stimulation and differentiates into internalized and surface-resident synaptic vesicles. The surface fraction was tagged during application of TTX on $4^{\circ} \mathrm{C}$. The spontaneously releasing population was derived from this condition and tagging during application of TTX on $37^{\circ} \mathrm{C}$. The size of the spontaneously releasing population is negligible in mature neurons. The inactive population of vesicles remains unlabelled during live-tagging and was revealed by an additional staining after fixation and permeabilization $(n=3$ independent experiments per data point, at least 10 neurons sampled per experiment). All data represent the mean \pm SEM.

A

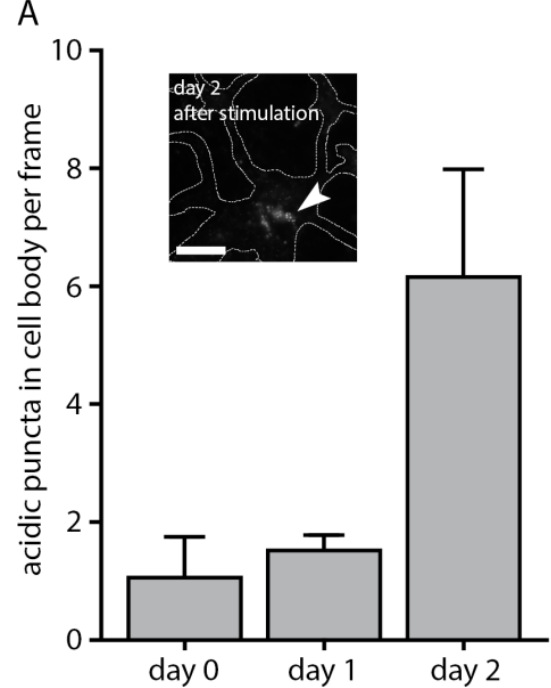

B

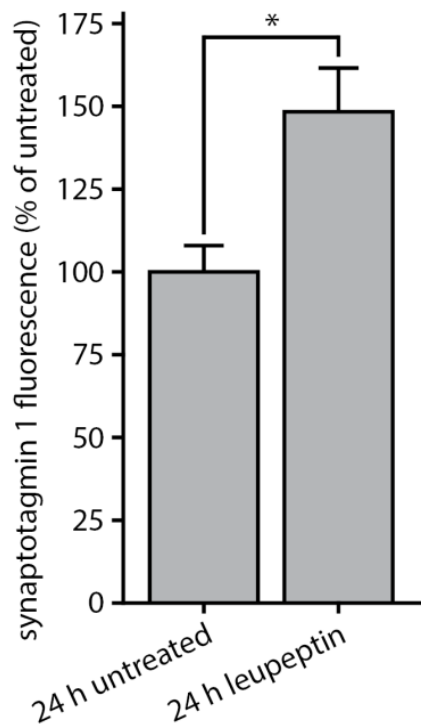

Figure 3.3: Live-tagged synaptic vesicles recapitulate the normal vesicle life cycle, including degradation in lysosomes.

(A) Synaptotagmin 1 live-tagged with CypHer5E appears in the cell body as non-releasable puncta (lysosomes) after $\sim 2$ days $(n=4,3,4$ independent experiments per respective time point, at least 12 neurons sampled per experiment). Inset: non-releasable tagged synaptotagmin 1 proteins in acidic compartments (indicated by the

arrow) inside the cell body (outlined with dashed white line) of a neuron 2 days after tagging and after stimulation (600 action potentials, $20 \mathrm{~Hz}$ ). Scale bar: $10 \mu \mathrm{m}$.

(B) Inhibiting lysosome function with leupeptin leads to a delay in degradation ( $n=3$ independent experiments per data point, at least 10 neurons sampled per experiment).

All data represent the mean \pm SEM. ${ }^{*} p<0.05$ 


\subsection{Synaptic vesicles lose the ability to release as they age}

The live-tagging approach characterized above can be used to track the degradation of synaptic vesicles, which follows a similar time course for synaptotagmin 1 and VGAT (Figure $3.4 A, B)$. Both proteins show an approximate half-life of 2-4 days. This is in agreement with previous descriptions of synaptic vesicle protein life-times by mass spectrometry and radioisotopic labelling (Cohen et al., 2013; Daly and Ziff, 1997).

Synaptic vesicle inactivation under high-frequency stimulation. Further, the functional state of synaptic vesicles can be monitored by using antibodies coupled to the $\mathrm{pH}$-sensitive fluorophore CypHer5E. CypHer5E is brightly fluorescent in $\mathrm{pH} 5.5$ inside synaptic vesicles and gets quenched upon release of the vesicles and contact of the vesicle lumen to the $\mathrm{pH}$ 7.4 extracellular medium (Hua et al., 2011a; Martens et al., 2008). A stimulation protocol of 600 action potentials triggered at $20 \mathrm{~Hz}$ leads, to evoke the release of all synaptic vesicles that are potentially releasable, was employed here to determine the maximally possible response of the ageing vesicles remaining at the synapse after up to 10 days. Both for livetagged synaptotagmin 1 and VGAT, releasable vesicles become progressively unresponsive to stimulation as they get older (Figure 3.4C,D). The inactivation proceeds somewhat faster for VGAT, presumably due to cell-type specific behaviour of the $\sim 5-10 \%$ minority of inhibitory neurons in the culture, but follows a similar dynamic for both proteins and reaches an absolute unresponsiveness at 7-10 days after tagging. This indicates two things: first, ageing synaptic vesicles progressively lose their ability to release in response to stimulation. Second, synaptic vesicle proteins age together, as an absolute inactivation could never be reached if the synaptic vesicles dispersed and were re-formed from old and young proteins during each round of release and recycling. The young proteins would rejuvenate the vesicle and the old proteins would continue to release and to recycle together with the young proteins that replenished the newly formed vesicle. This provides further evidence that the synaptic vesicle maintains its integrity during release and recycling (see also Chapter 1.4 in the Introduction). 

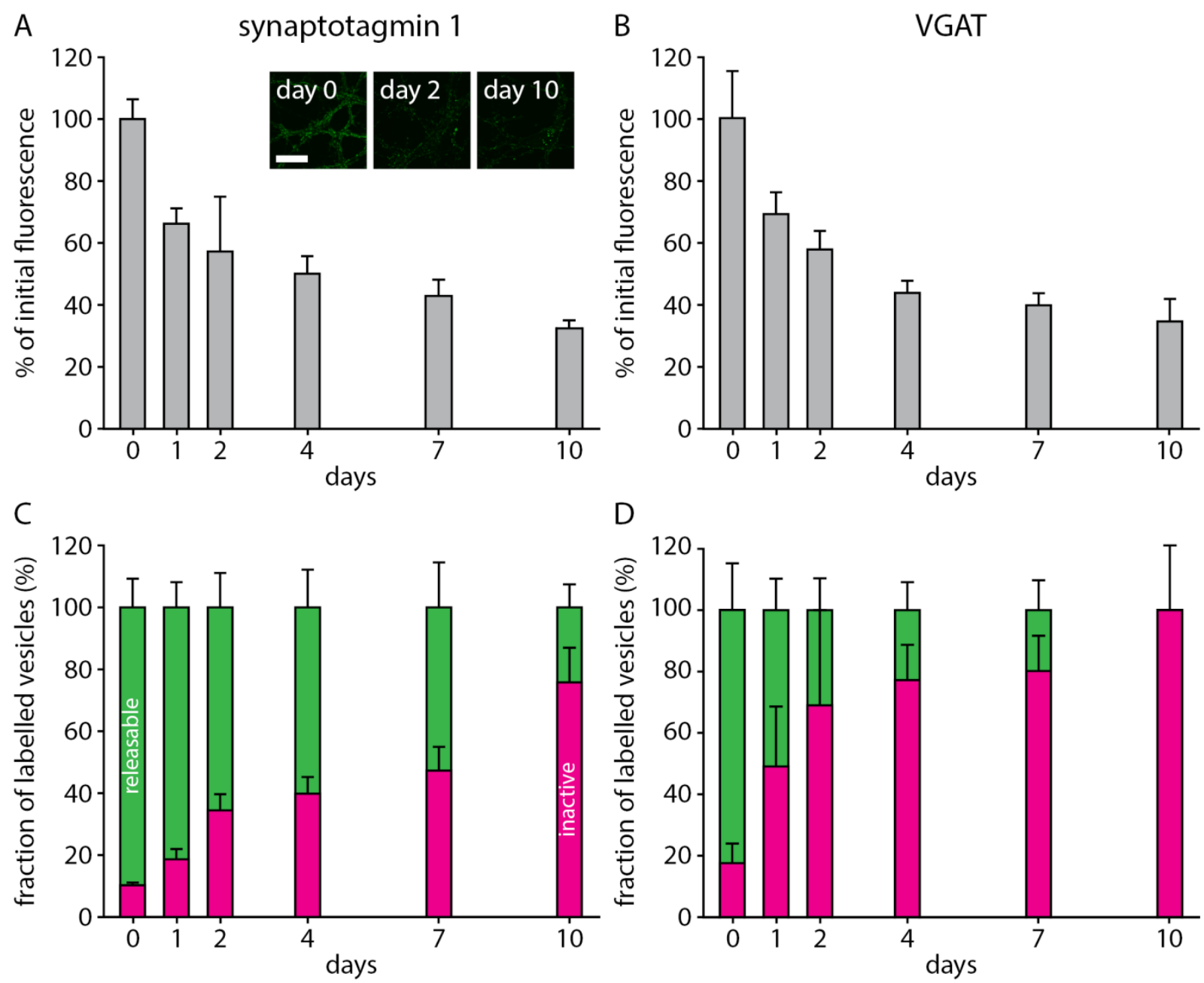

Figure 3.4: Synaptic vesicles lose the ability to release as they age.

(A-B) The loss of synaptic vesicles was monitored by imaging the antibody fluorescence at serial time points after tagging synaptotagmin $1(A ; n=3,3,2,3,3,2$ independent experiments per respective time point, at least 10 neurons sampled per experiment) or VGAT (B; $n=3,4,2,4,4,3$ independent experiments per respective time point, at least 10 neurons sampled per experiment). Inset: exemplary images of synapses labelled with synaptotagmin 1 antibodies. Scale bar: $20 \mu \mathrm{m}$. (C-D) Synaptic vesicle responses to stimulation (600 action potentials at $20 \mathrm{~Hz}$ ), monitored by imaging antibodies conjugated to the $\mathrm{pH}$-sensitive fluorophore CypHer5E. The antibodies were directed against synaptotagmin 1 ( $C ; n=4,3,4,4,2$, 2 independent experiments per respective time point, at least 12 neurons sampled per experiment) or VGAT ( $D ; n=3,4,2,4,4$, 3 independent experiments per respective time point, at least 8 neurons sampled per experiment). All data represent the mean \pm SEM. 
Synaptic vesicle inactivation during intrinsic network activity. Since these observations are based on supra-physiological stimulation (600 action potentials at $20 \mathrm{~Hz}$ ), I wanted to observe the inactivation of ageing synaptic vesicles during intrinsic network activity of the cultures. To do this, I used an unconjugated version of the synaptotagmin 1 antibody to tag releasable vesicles and followed this up with pulses of fluorescently conjugated secondary antibodies at subsequent time points, to reveal the vesicles that are still releasable (Figure 3.5A). To obtain a line of comparison, I revealed the total population of remaining tagged synaptic vesicles by fixing and permeabilizing the neurons, followed by staining with a second secondary antibody conjugated to a spectrally separable fluorophore (Figure 3.5A). This experiment revealed that releasable synaptic vesicles become inactive and unresponsive to stimulation after less than a day ( $T=0.5$ days; Figure $3.5 B, C)$, substantially faster than when applying artificial stimulation (Figure 3.4C,D).

A

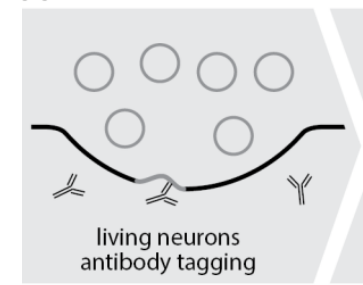

B
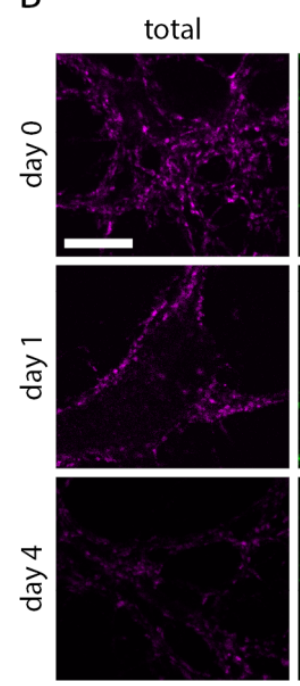

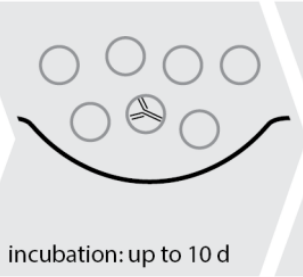

C
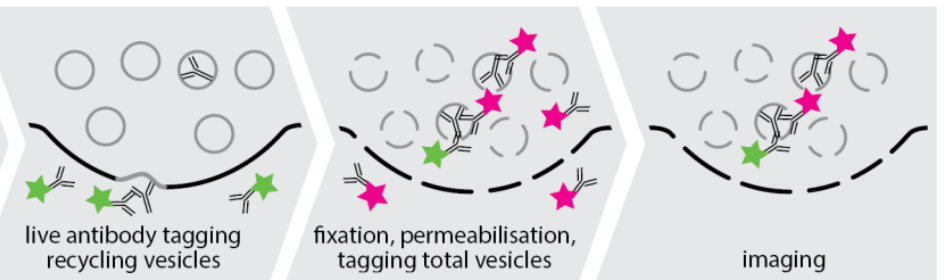
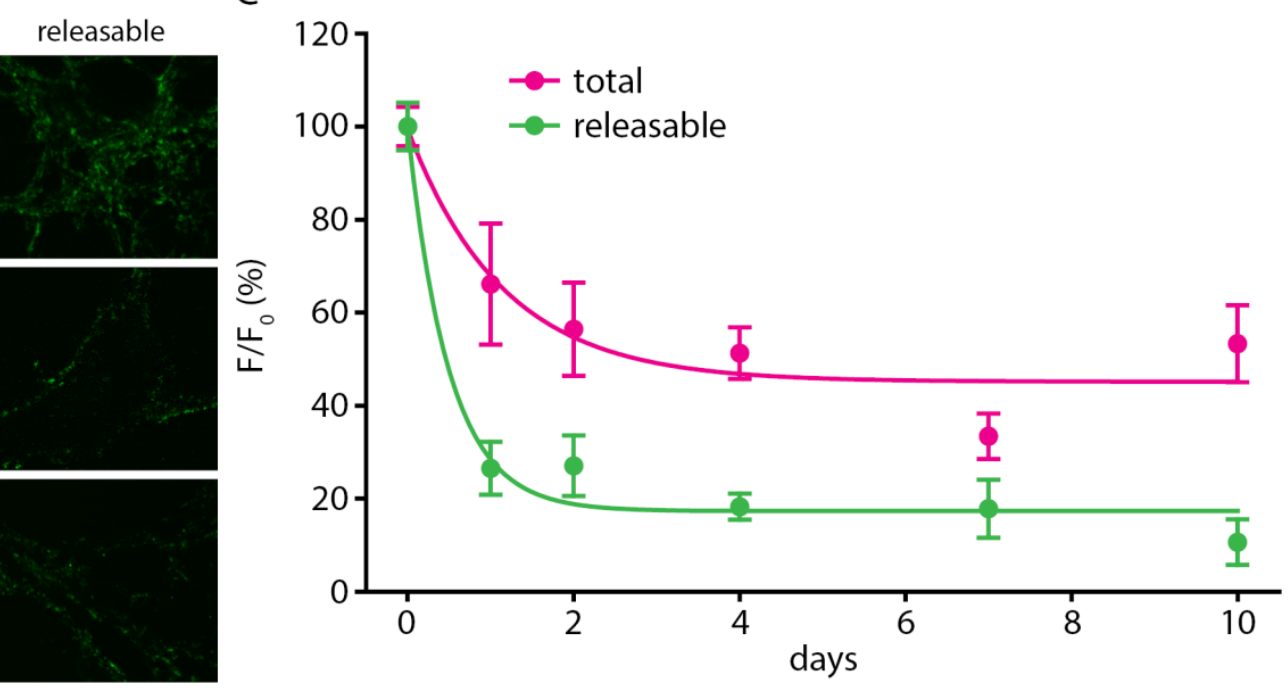

Figure 3.5: Synaptic vesicles become inactive within less than a day during intrinsic network activity.

(A) Schematic of the experiment: releasable synaptic vesicles were tagged with unconjugated antibodies against synaptotagmin 1 . These tags were then revealed on vesicles that continue to release by a fluorescently conjugated secondary antibody added to the culture medium of the neuron cultures (green). To obtain a line of comparison, neurons were then fixed and permeabilized, and a second secondary antibody conjugated to a spectrally separable fluorophore was applied to reveal all initially tagged synaptic vesicles still left (pink).

(B) Exemplary images of loss of tagged vesicles and inactivation of releasable vesicles. Scale bar: 10 $\mu \mathrm{m}$. 
(C) Quantification of loss of tagged vesicles from the synapse and inactivation of releasable vesicles ( $n=5,4,4,3,3,2$ independent experiments per respective time point, at least 10 neurons sampled per experiment). The degradation of vesicles is identical to what is shown by similar means in Figure 3.4A. The lines are best fits obtained from 3-parameter exponential decay functions. The time constant T of inactivation is $\sim 0.5$ days.

All data represent the mean $\pm \mathrm{SEM}$.

\section{Synaptic vesicle inactivation assessed with a novel genetic construct. Since all} experiments presented so far are based on antibody live-tagging, and although this is a robust approach (Figure 3.1), I wanted to confirm the observations made so far with an alternative technique. For this, I utilized a novel genetic construct: VAMP2-TEV-SNAPtag. This consists of synaptobrevin/VAMP2, fused to a TEV protease cleavage site at the vesicle lumenal C-terminus, followed by a SNAP-tag (Figure 3.6A). The construct targets to synaptic vesicles via VAMP2, which has been used successfully to robustly target indicators of vesicle function to synaptic vesicles in C-terminal fusions before (Gandhi and Stevens, 2003; Miesenböck et al., 1998). The SNAP-tag self-catalyses the covalent attachment of fluorescent dyes with appropriate reactive groups (Keppler et al., 2003, 2004), which can be used to label synaptic vesicles in living neurons with cell-permeable fluorophores (Juillerat et al., 2003). To separately tag synaptic vesicles of different ages, I first applied one pulse of fluorophores and then waited 1-2 days before applying a second pulse with a different spectrally separable fluorophore. The first pulse was saturating (data not shown). The TEV protease cleavage site in the construct could then be used to cleave off the fluorescent tag from old or young vesicles during intrinsic network activity of the cultures during live imaging (Figure 3.6B). This allows differentiating which synaptic vesicles are preferentially used in release, the young or the old ones. The loss of fluorescence from young synaptic vesicles was indeed higher than the loss of fluorescence from the old vesicles tagged 1-2 days earlier (Figure 3.6C). This confirms the previous antibody-based observations.

I could thus establish so far, using antibody live-tagging or genetic timer constructs, and for three different synaptic vesicle proteins (synaptotagmin 1, synaptobrevin/VAMP2, VGAT), that releasable synaptic vesicles become inactive as they age. Again, this incidentally also confirms that synaptic vesicles stay largely intact during release and recycling, since otherwise observations on functional changes with age would not be possible due to an intermixing of synaptic vesicle proteins of different ages, leading to an equilibration of the age of the synaptic vesicle as a whole (also see Chapter 1.4). 
A

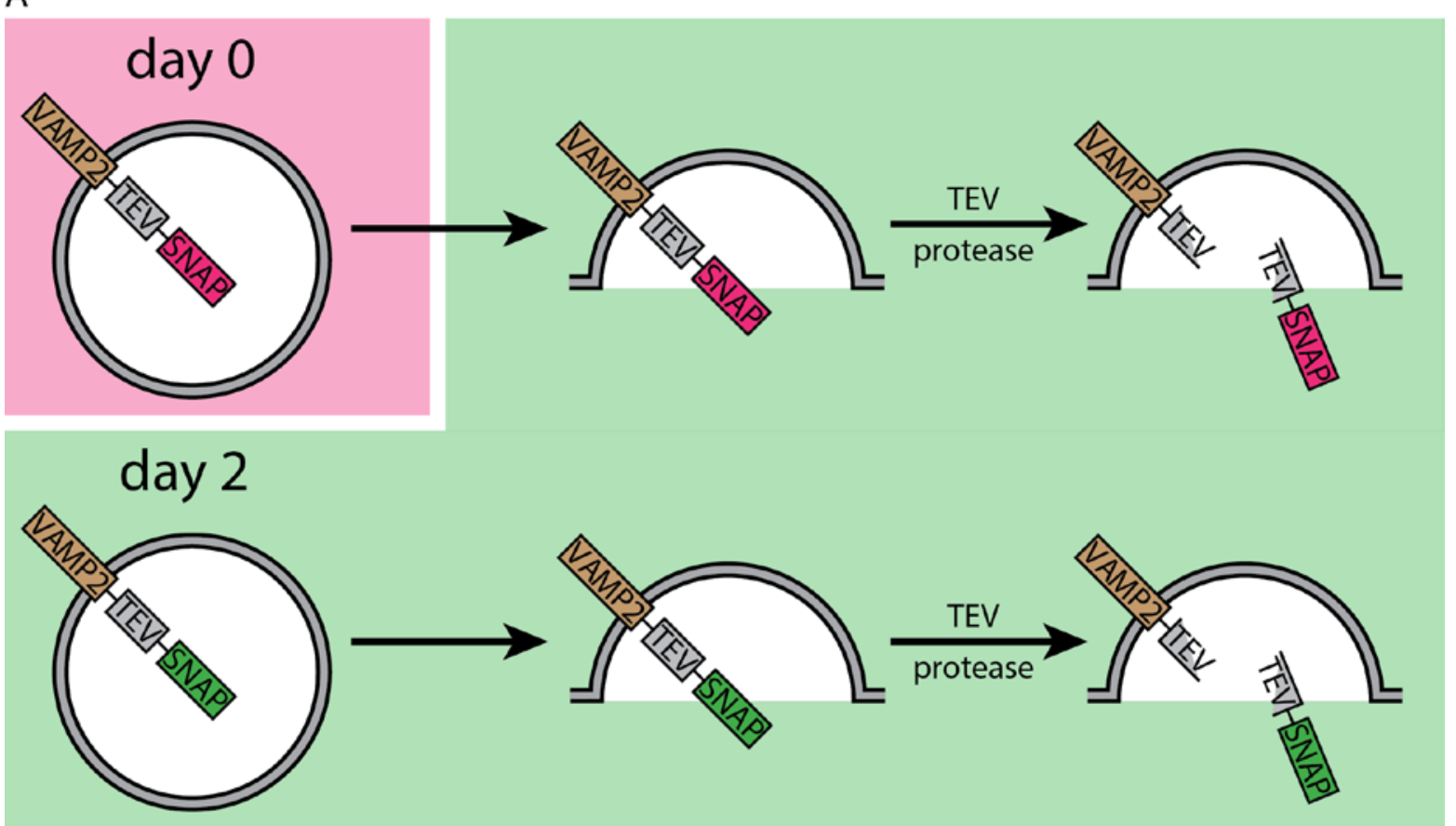

B
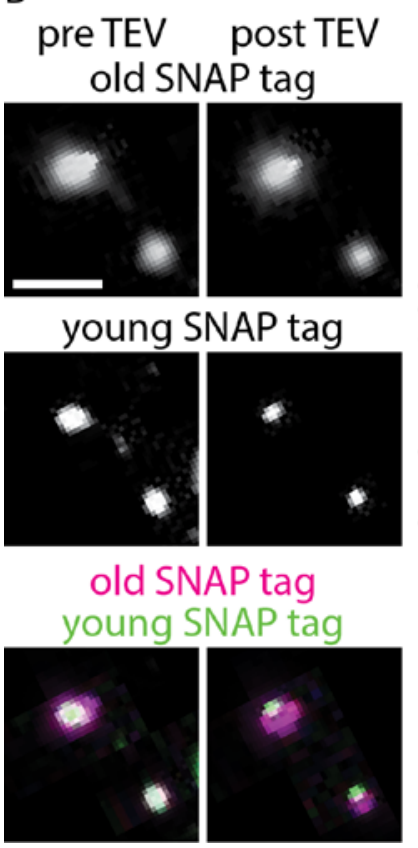

C
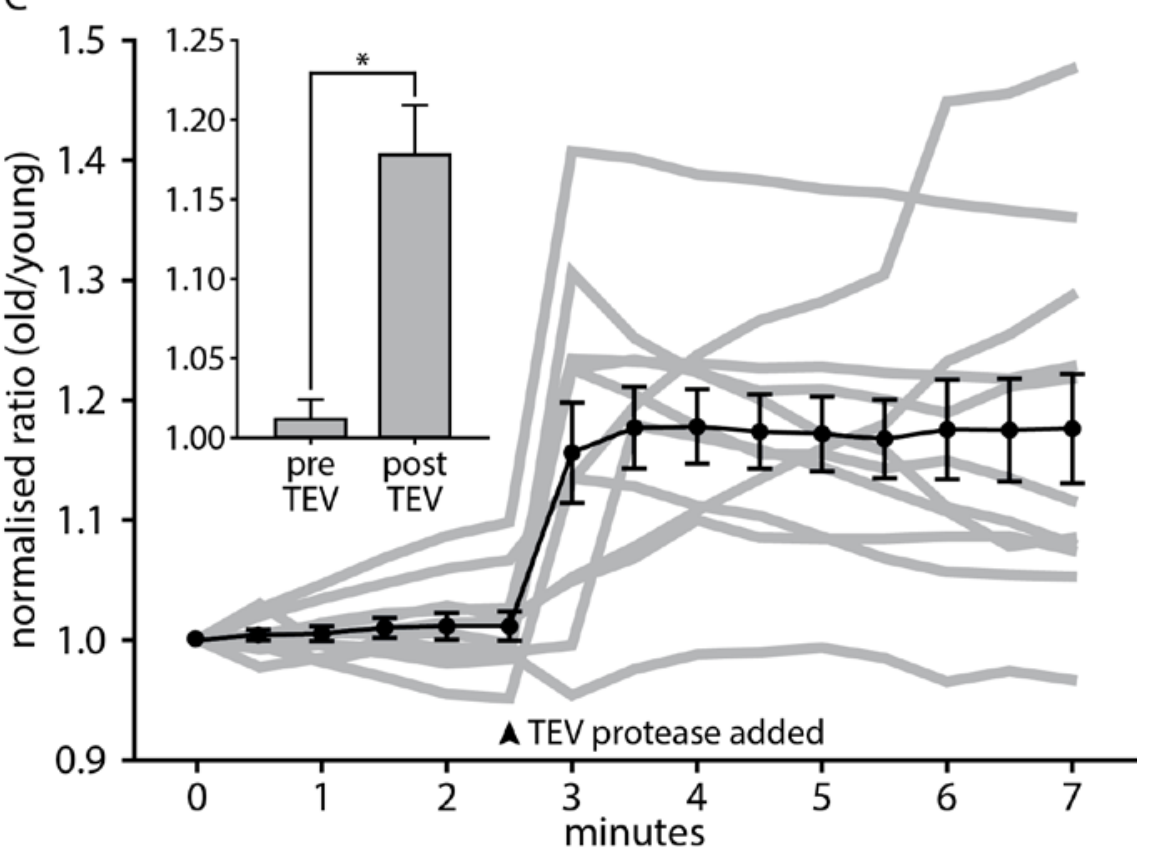

Figure 3.6: A novel VAMP2-based genetic reporter of synaptic vesicle ageing confirms that young synaptic vesicles are preferentially released.

(A) Schematic of the experiment: a VAMP2-TEV-SNAPtag construct was expressed in neurons and then labelled first with a saturating pulse of TMR-Star (pink) and then, after 1-2 days, with a second pulse of the spectrally separable 647-SiR (green). Following the second labelling pulse, neurons were live-imaged in both channels and TEV protease was applied during imaging to determine whether the tag on the old or the young vesicles was preferentially cleaved.

(B) Exemplary images of two synapses with young and old vesicles labelled via the VAMP2-TEVSNAPtag construct, before and after TEV protease cleavage. Scale bar: $2 \mu \mathrm{m}$.

(C) A quantification of the ratio of old over young synaptic vesicle label during imaging. Application of the TEV protease is indicated by the arrow at $2.5 \mathrm{~min}$. The neurons were allowed to be active under 
their intrinsic network activity. The grey traces show individual experiments, which reflect the different activity levels of the sampled neurons. The black trace is the mean \pm SEM ( $n=11$ independent experiments). Inset: ratio directly before application of TEV protease and at the highest ratio reached during the experiment after application of TEV protease.

All data represent the mean \pm SEM. ${ }^{*} p<0.05$

\subsection{Releasable synaptic vesicles are metabolically young and the synapse is dependent on replenishment with young vesicles}

Having established that synaptic vesicles lose the ability to release as they age, I next wanted to establish if the vesicles used in release are also metabolically younger than other vesicles. A previous study found that synaptic vesicle proteins detected on bona fide synaptic vesicles seem to be generally younger than the same proteins found on endosomes that are presumably entering the endolysosomal degradation pathway (Fernandes et al., 2014a), but this does not assert anything about the age and functionality of the synaptic vesicles before they enter the degradation pathway, the last step in their life cycle (see Chapter 1.5). The experiments presented so far in this Thesis suggest that young synaptic vesicles are more active and releasable (Figure 3.4, Figure 3.5, Figure 3.6), but are not sufficient to positively assert it. I approached this problem via two independent methods of metabolic labelling. In both approaches, I tagged recently produced proteins, then identified releasable synaptic vesicles by live-tagging, and finally imaged the degree of co-localisation between the tags for releasable vesicles and young proteins. To perform the same experiment for inactive vesicles, I reversed the sequence of live-tagging and metabolic labelling and waited 4 days in between to allow the tagged vesicles to become inactive (see Figure 3.7A for a schematic of the experiment).

FUNCAT. In one approach, I used the artificial amino acid L-azidohomoalanine (AHA) for metabolic labelling. AHA is incorporated in the position of methionine into all proteins newly produced during its incubation with the cultures. AHA can subsequently be detected in fluorescence microscopy after fluorophore conjugation (FUNCAT; Dieterich et al., 2011) through CLICK chemistry after fixation (Rostovtsev et al., 2002). This allows simple correlative imaging of the AHA label of recently produced proteins and releasable or inactive vesicles live-tagged with fluorophore-conjugated antibodies. Using two-colour STED microscopy on ultrathin sections allowed single vesicle resolution in this approach. This revealed that newly produced proteins co-localize significantly more with releasable synaptic vesicles than with inactive ones (Figure 3.7B). 
COIN. In another approach, I used ${ }^{15} \mathrm{~N}$ leucine (instead of AHA) as a metabolic label. ${ }^{15} \mathrm{~N}$ is a stable isotope of nitrogen that only appears at a frequency of less than $0.4 \%$ in nature and can thus be used as a tag in this experiment. To identify the tag in the sample, I turned to nanoSIMS, a mass spectrometry imaging technique (Lechene et al., 2006; Steinhauser and Lechene, 2013). The co-localization with the fluorescent tag of releasable synaptic vesicles was determined through prior fluorescence imaging (COIN; Saka et al., 2014b). This allowed me to determine the ${ }^{15} \mathrm{~N} /{ }^{14} \mathrm{~N}$ ratio, as an indicator of protein age, of protein material associated with releasable synaptic vesicles and inactive synaptic vesicles. Furthermore, the nanoSIMS approach allows to compare this with the ${ }^{15} \mathrm{~N} /{ }^{14} \mathrm{~N}$ ratio in adjacent axonal segments, as a baseline for the average protein age in the cell, as the axon is a compartment of active retrograde and anterograde trafficking and thus an intermixing of cellular structures with different ages. I found that the releasable vesicles are significantly younger than the axon $(p<0.0005)$, and that the inactive vesicles are significantly older $(p<$ 0.0001 ), while the releasable vesicles are also significantly younger than the inactive vesicles $(p<0.00001$; Figure 3.7C). This further strengthens the previous observation that young synaptic vesicles are preferentially used in release. 
A

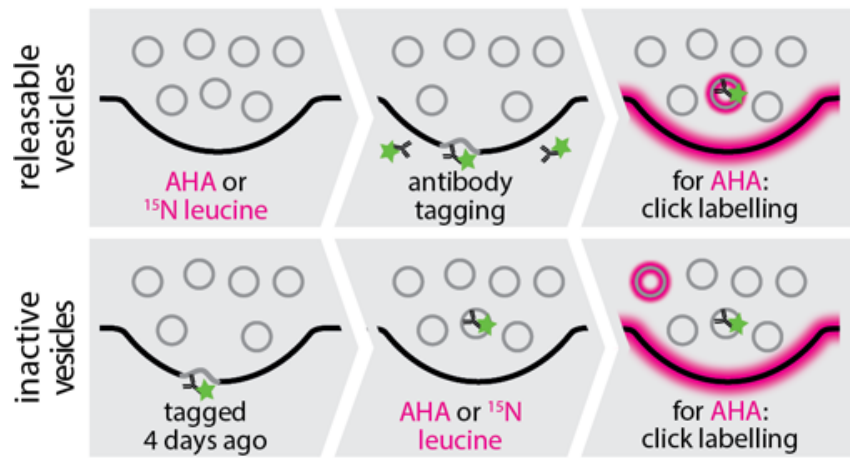

B

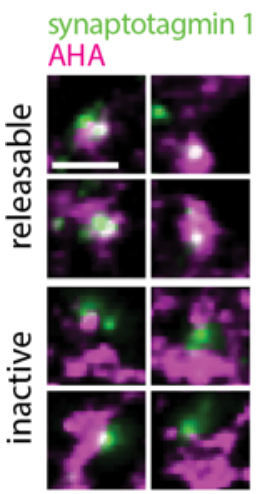

C

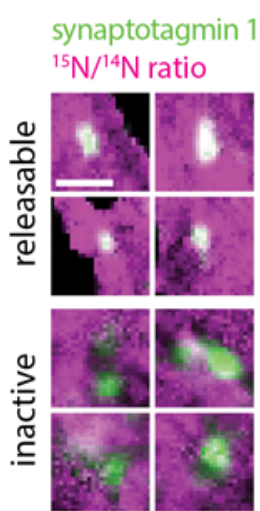

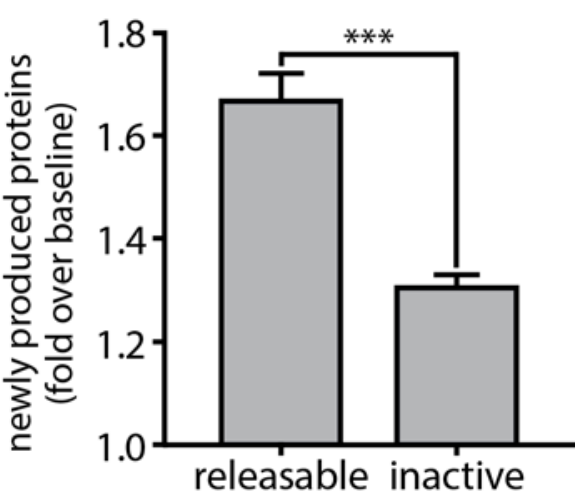

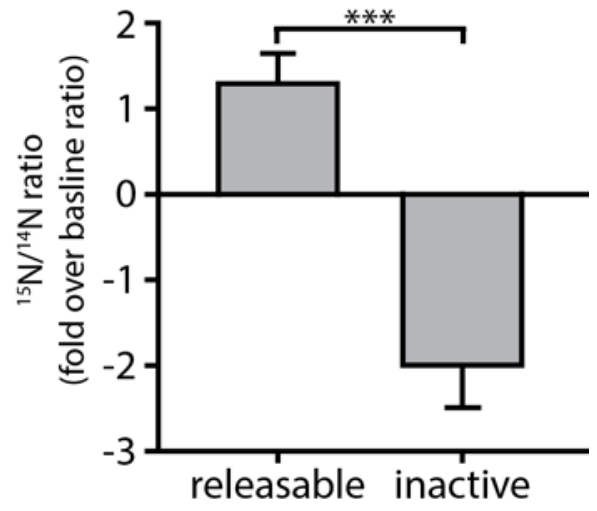

Figure 3.7: Releasable synaptic vesicles are metabolically young.

(A) Schematic of the experiment: for metabolic labelling of recently produced proteins, I applied either AHA or ${ }^{15} \mathrm{~N}$ leucine. Antibodies were then applied to tag releasable synaptic vesicles, followed by imaging both labels to determine their colocalization (upper panel). To determine the co-localization of inactive synaptic vesicles with newly produced proteins, the antibody tag was applied first and the metabolic label was added after 4 days (lower panel), when the vesicles became inactive (see Figure 3.5).

(B) Co-localization of the metabolic AHA label of recently produced proteins with releasable and inactive synaptic vesicles in STED microscopy on $50 \mathrm{~nm}$ thin sections $(\mathrm{n}=3$ independent experiments per data point, at least 10 neurons sampled per experiment). Left: exemplary images. Scale bar: $500 \mathrm{~nm}$. (C) ${ }^{15} \mathrm{~N} /{ }^{14} \mathrm{~N}$ ratio of releasable and inactive synaptic vesicles in correlated fluorescence imaging and nanoSIMS $(n=57$ synapses from 3 independent experiment for releasable synaptic vesicles, $n=47$ synapses from 2 independent experiments for inactive

vesicles). The ratio is given as fold over the ${ }^{15} \mathrm{~N} /{ }^{14} \mathrm{~N}$ ratio of the axonal regions neighbouring the synapses (baseline). Inactive vesicles contain substantially fewer newly synthesized proteins than the rest of the axon $(p<0.0001)$, while the active vesicles contain substantially more newly synthesized proteins $(p<0.0005)$. Left: exemplary images. Scale bar: $500 \mathrm{~nm}$.

All data represent the mean \pm SEM. ${ }^{* *} p<0.005$ 
Dependence of the synapse on young synaptic vesicles. I next set out to complement this information on protein age with a functional approach, to determine if the synapse is also dependent on the use of young vesicles or if older vesicles could be reactivated to substitute for young vesicles in release. To do this, tracked new synaptic vesicles entering the releasable population by blocking the epitopes on all releasable vesicles with an unconjugated version of the monoclonal antibody against synaptotagmin 1 used throughout this thesis, followed by sequential pulses with the same antibody, but conjugated to a fluorophore (see Figure 3.8A for a schematic of this approach). This reveals new vesicles with unblocked epitopes. To test whether the synapse could replace the releasable population from old, inactive vesicles residing locally at the synapse ( $\sim 50 \%$ of all vesicles at the synapse, see Figure 3.2), instead of young vesicles recently arrived at the synapse, I cut off the supply of newly produced vesicles to the synapse. To do this, I applied anisomycin to block protein biosynthesis, or colchicine to disrupt the transport of newly synthesized synaptic vesicles from the cell body to the synapse. In both cases, the synapse was unable to recruit old inactive vesicles and no replacement of the releasable population occurred, while replacement was clearly observable in an untreated control condition where access to newly produced synaptic vesicles was unrestricted (Figure 3.8B,C). The drugs used did not significantly impair release of synaptic vesicles in response to stimulation (Figure 3.8D), or during intrinsic network activity (Figure 3.8E), and did not change the total vesicle pool size at the synapse (Figure 3.8F) throughout the time of the experiment. 
A

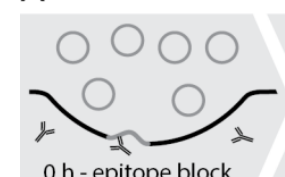

$0 \mathrm{~h}$ - epitope block

B
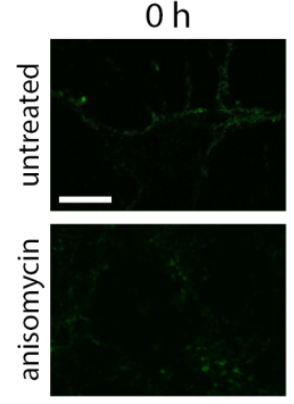

$\mathrm{D}$

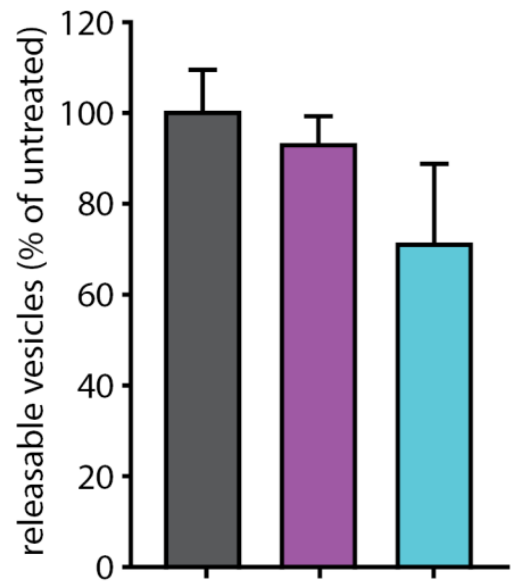

untreated anisomy colchicine

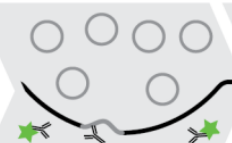

$\mathrm{o} \mathrm{h}$-no fluorescence

C

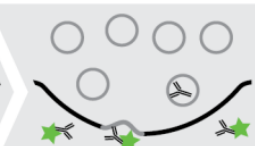

$24 \mathrm{~h}$ - new epitopes

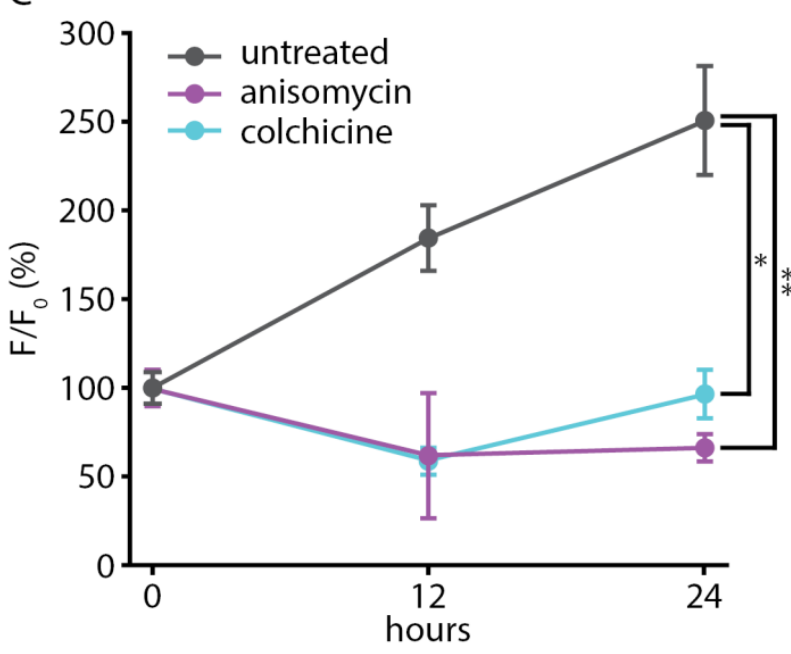

$\mathrm{F}$
$\mathrm{E}$

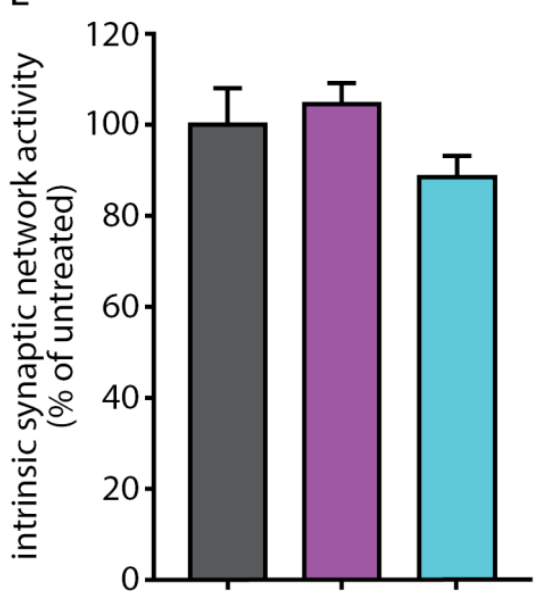

untreated anisomy colchicine

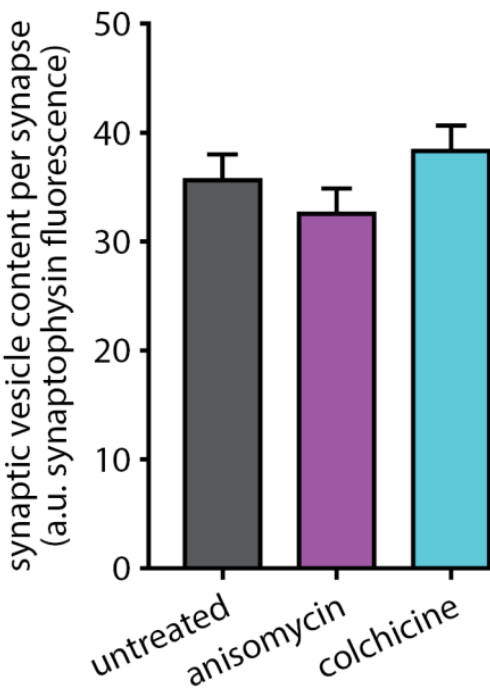

Figure 3.8: The synapse is critically dependent on newly produced synaptic vesicles to replenish the releasable population.

(A) Schematic of the experiment: epitopes of releasable synaptic vesicles can be blocked with an unconjugated monoclonal synaptotagmin 1 antibody, and new synaptic vesicles entering the releasable population can subsequently be revealed at serial time points by applying a fluorophoreconjugated version of the same antibody.

(B) Exemplary images of new synaptic vesicles entering the releasable population under untreated conditions and during inhibition of protein biosynthesis via anisomycin. Scale bar: $10 \mu \mathrm{m}$.

(C) The releasable population of synaptic vesicles is replaced only when the synapse has access to newly produced synaptic vesicles (untreated). When protein biosynthesis was suppressed (anisomycin) or the transport of newly synthesized synaptic vesicles was disrupted (colchicine), there was no replacement of the releasable population of synaptic vesicles $(n=3-4$ independent experiments per data point, at least 10 neurons sampled per experiment).

(D) The drugs used to cut the synapse off from newly produced proteins did not significantly impair the release of synaptic vesicles in response to stimulation (600 action potentials, $20 \mathrm{~Hz}$ ). The drugs were applied for $24 \mathrm{~h}$, as in the experiments shown in (B-C), before stimulation in the presence of 
fluorophore-conjugated synaptotagmin 1 antibodies ( $n=3$ independent experiments per data point, at least 10 neurons sampled per experiment).

(E) The drugs did not affect the intrinsic network activity of cultures after $24 \mathrm{~h}$ of treatment, as measured via uptake of synaptotagmin 1 antibodies ( $n=3$ independent experiments per condition).

(F) The total size of the synaptic vesicle pool was not affected, as determined by synaptophysin immunostaining after $24 \mathrm{~h}$ of drug treatment ( $\mathrm{n}=3$ independent experiments per condition).

All data represent the mean \pm SEM. ${ }^{*} p<0.05,{ }^{* \star} p<0.01$

\subsection{Synaptic vesicles release $\sim 260$ times before becoming inactive} Young synaptic vesicles are active and releasable, aged synaptic vesicles are inactive and cannot replenish the releasable population even when the synapse is cut off from newly produced vesicles (Figure 3.8). This indicates that there is a mechanism that specifically prevents the use of aged synaptic vesicles or that aged synaptic vesicles are intrinsically less releasable. It is difficult to make sense of this observation by ascribing it exclusively to vesicle age, however. Damage or other molecular changes can usually only occur to an organelle if it is getting used or if it resides in a metabolically highly active environment (Burré et al., 2006b; Dobson, 1999, 2003; Matouschek, 2003; Prakash and Matouschek, 2004; see Chapter 4.4 in the Discussion for an in-depth treatment of this aspect). For the synaptic vesicle, use means fusion to the cell membrane (Südhof, 2004) and, possibly, recycling through endosomal compartments (Jähne et al., 2015; Rizzoli, 2014), which could lead to changes in the molecular composition of the vesicle. I therefore set out to determine if the inactivation of ageing synaptic vesicles might be related to their usage frequency and the activity of the synapse rather than to their temporal age alone.

Increased synaptic activity frequency accelerates vesicle inactivation. To do this, I repeated the experiment from Figure 3.5, but while increasing the release probability of synaptic vesicles during intrinsic network activity over a period of $12 \mathrm{~h}$. To do this, I used either the $\mathrm{GABA}_{\mathrm{A}}$ receptor antagonist bicuculline to silence inhibitory signalling, or a calcium concentration raised to $8 \mathrm{mM}$ to lower the threshold of stimulation. In both cases, releasable synaptic vesicles became inactive significantly sooner (Figure 3.9). 
A

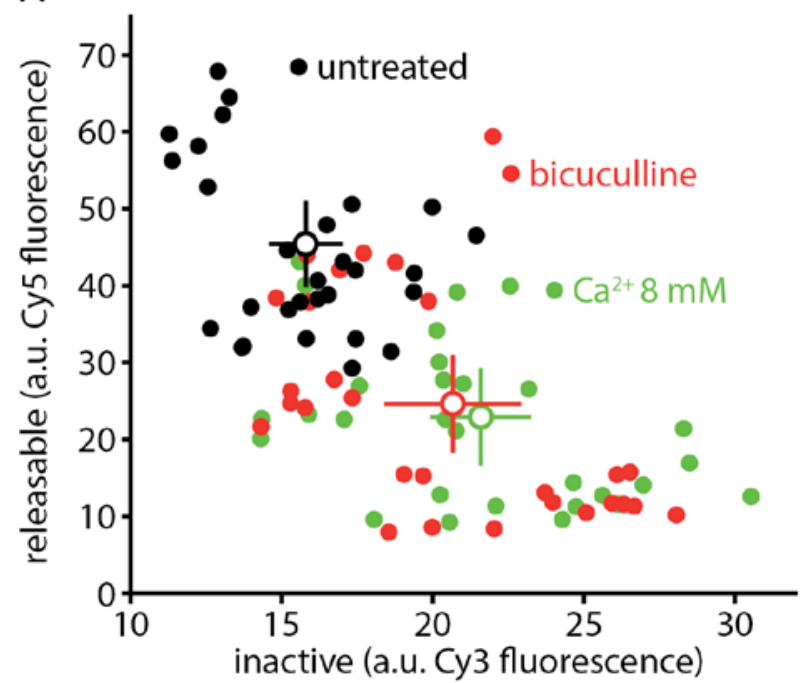

B

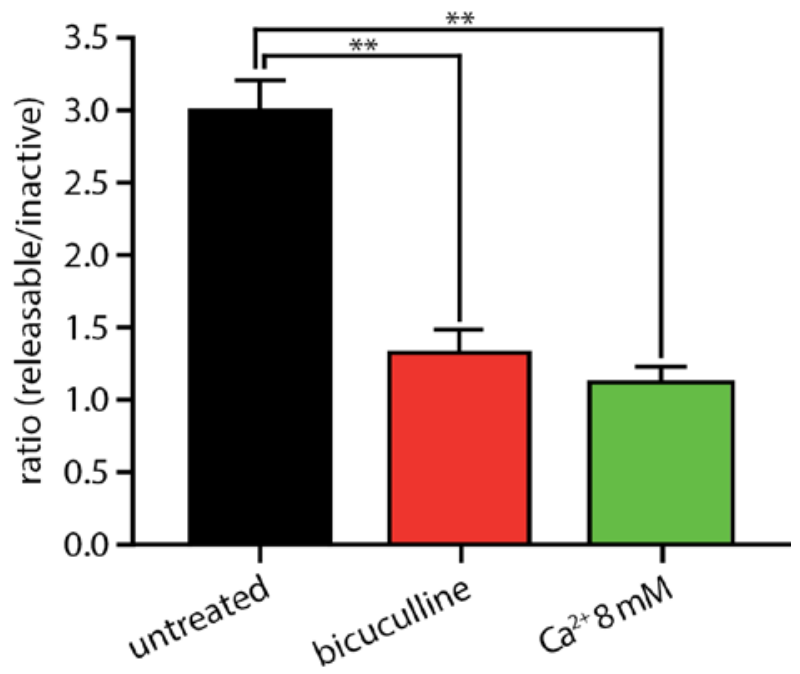

Figure 3.9: Synaptic vesicle inactivation is not determined by passing time, but by usage.

(A) To determine whether increased activity speeds up inactivation of synaptic vesicles, the experiment shown in Figure 3.5 was repeated after $12 \mathrm{~h}$ of treatment with bicuculline (blocks inhibitory $\mathrm{GAGA}_{\mathrm{A}}$ receptors) or raising the $\mathrm{Ca}^{2+}$ concentration to $8 \mathrm{mM}$ (increases probability of vesicle release), with untreated medium as control ( $n=3$ independent experiments per condition, at least 10 neurons sampled per experiment). The intensity of signal from releasable and inactive vesicles is shown (filled dots for individual experiments, empty circles with error bars for the mean $\pm S E M$ ).

(B) Quantification of the ratio of releasable over inactive vesicles from (A).

All data represent the mean \pm SEM. ${ }^{* *} p<0.01$

Dual measurement of synaptic activity and release. After having determined that releasable synaptic vesicles are metabolically young (Figure 3.7) and lose their ability to release as they age (Figure 3.4, Figure 3.5, Figure 3.6), and further determining that the synapse is dependent on a constant supply of newly produced synaptic vesicles to replenish its releasable population (Figure 3.8), which seems to be limited to a certain number of release events per vesicle (Figure 3.9), I wanted to determine how often a synaptic vesicle could be employed in release and be recycled before it becomes inactive. To calculate that number, only three parameters are needed: (1) the time a synaptic vesicle spends in the releasable population at the synapse (already determined in Figure $3.5 \mathrm{C}, \mathrm{T}=0.5$ days), (2) the frequency of synaptic activity of neurons in culture, and (3) the fraction of releasable vesicles released per activity burst (see Equation (1) in the following section). To determine the missing parameters experimentally, I used a combination of calcium indicator imaging (GCaMP6; Chen et al., 2013) and concurrent imaging of a reporter of synaptic vesicle release (synaptophysin-based pHluorin sypHy; Granseth et al., 2006; Li and Murthy, 2001). First, imaging $\mathrm{Ca}^{2+}$ spikes during stimulation of neurons allowed me to calculate the intrinsic network activity frequency, which is $\sim 0.09 \mathrm{~Hz}$ (Figure 3.10A-C). But one spike of network activity does not correspond to one single action potential, but is composed to $\sim 60-80$, on 
average, as demonstrated by comparing the amplitude of intrinsic network activity bursts to spikes evoked by external stimulation of different amount (Figure 3.10D). Imaging $\mathrm{Ca}^{2+}$ spikes (GCaMP6) in parallel with synaptic vesicle release (sypHy) revealed that both robustly coincided (Figure 3.10E-G). I could then use this to determine how much of the releasable population of synaptic vesicles was released per activity burst, by comparison to release of the entire releasable population through stimulation with 600 action potentials at $20 \mathrm{~Hz}$ (Figure $3.10 \mathrm{H}, \mathrm{I}$ ); this was $-7.5 \%$, or $\sim 3 \%$ of the total synaptic vesicle population (Figure 3.10I). 


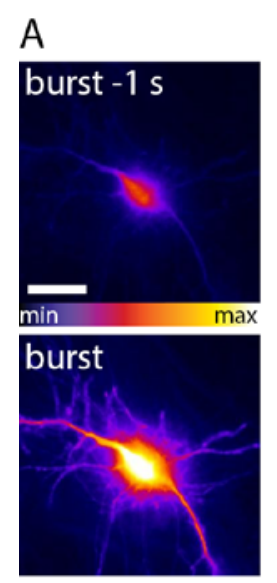

\section{B}

C
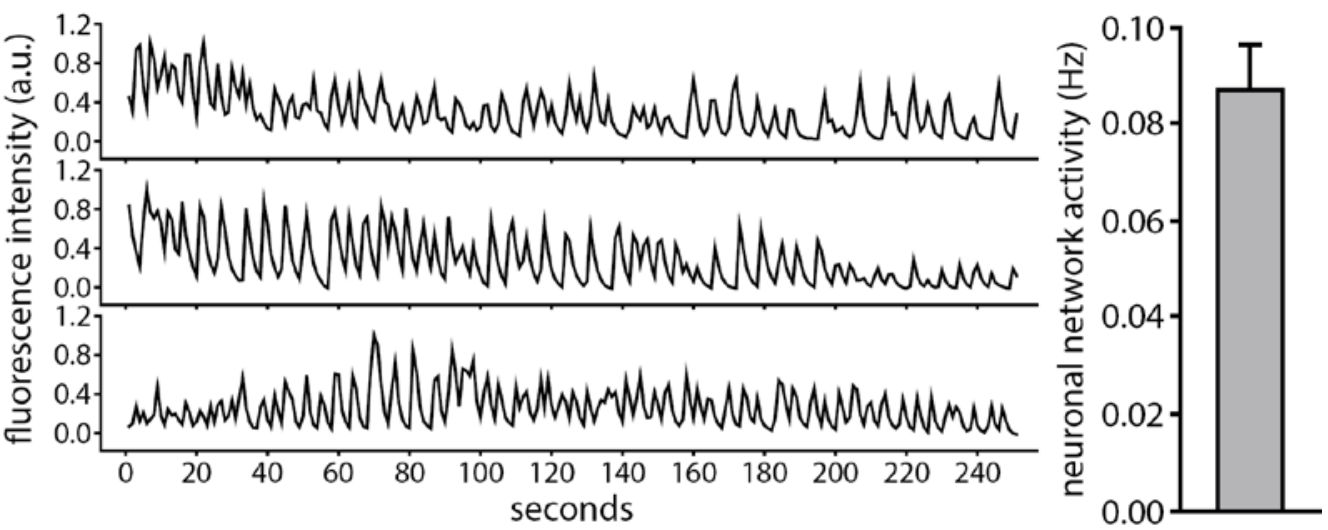

D
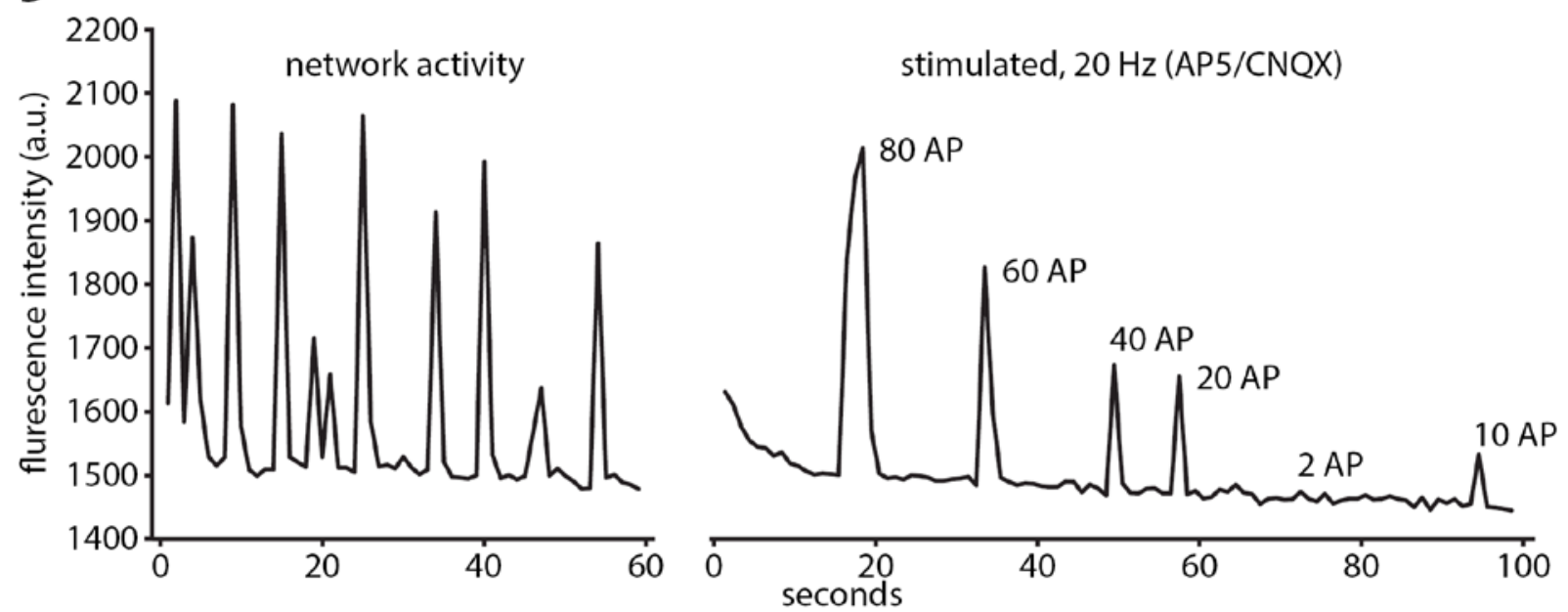

E

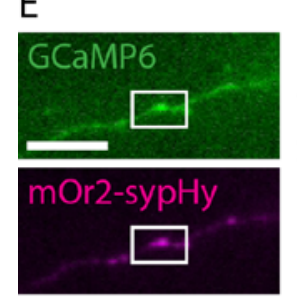

G

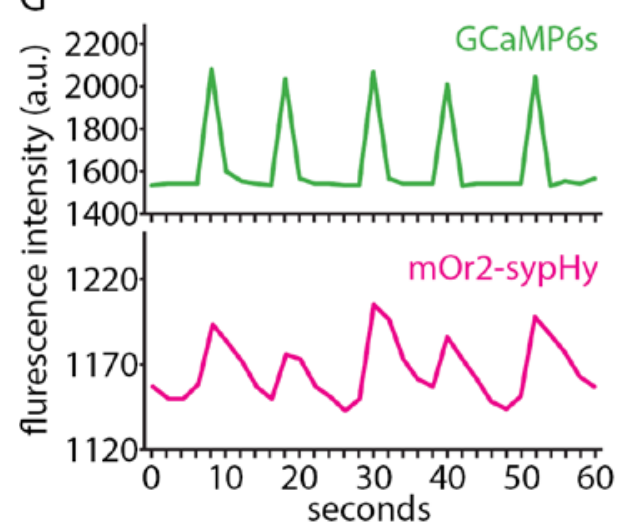

$\mathrm{F}$

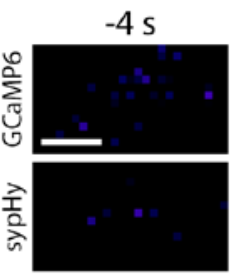

(1)

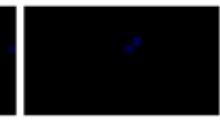

$\mathrm{H}$
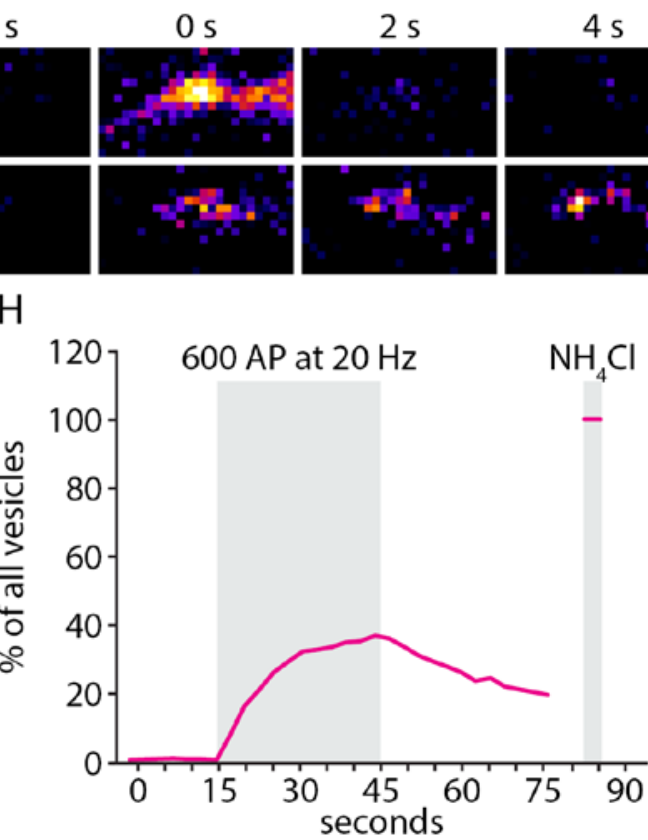

$2 s$
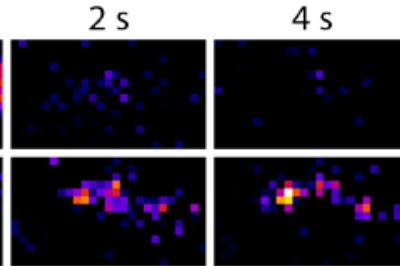

1

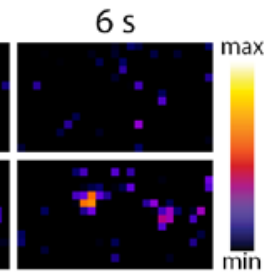

Figure 3.10: Live-cell imaging of synaptic activity and synaptic vesicle release allows the calculation of the number of release events per synaptic vesicle lifetime.

(A) Exemplary images of a neuron imaged with the calcium indicator GCaMP6 directly before and during an activity burst. Scale bar: $20 \mu \mathrm{m}$. 
(B) Exemplary traces of neuronal activity bursts during intrinsic network activity of the cultures, as revealed by GCaMP6 imaging.

(C) Quantification of the average frequency of activity bursts in hippocampal cultures.

(D) A quantitative comparison of the calcium signals evoked during intrinsic network activity (left), and during $20 \mathrm{~Hz}$ trains of electrical field stimulation (right). The stimulation trains were varied in duration to correspond to the indicated number of action potentials (AP). The comparison suggests that the bursts during intrinsic network consist of several tens of action potentials, usually $\sim 60-80$.

(E) Exemplary images of an axon with a synapse from a neuron co-transfected with GCaMP6 and sypHy (mOr2 variant to allow spectral separation from GCaMP6). Scale bar: $2 \mu \mathrm{m}$.

(F) Exemplary images of changes in GCaMP6 and sypHy fluorescence changes during activity bursts (area is the magnified white box from (E)). Scale bar: $500 \mathrm{~nm}$.

(G) Exemplary traces of fluorescence changes from GCaMP6 and sypHy during synaptic activity. The signals coincide robustly. The activity bursts are of approximately equal intensity (GCaMP6), but the amount of release per burst (sypHy) can vary.

$(\mathrm{H})$ An exemplary trace of synaptic vesicle release measured by sypHy during electrical field stimulation with 600 action potentials (AP) at $20 \mathrm{~Hz}$. For comparison, the total pool of vesicles (releasable and inactive) was assessed after stimulation with a pulse of $\mathrm{NH}_{4} \mathrm{Cl}$ (equilibrates $\mathrm{pH}$ in all intracellular compartments to neutral).

(I) Quantification of the fraction of synaptic vesicles released during a single activity burst (average for individual $\mathrm{Ca}^{2+}$ bursts derived from traces as shown in (G), average for 600 AP derived from traces as shown in $(\mathrm{H})$ ).

All data represent the mean \pm SEM.

Release events per vesicle lifetime: $\mathbf{2 6 0}$. The parameters derived from these experiments now allowed me to estimate the number of release events per synaptic vesicle lifetime that occur before inactivation:

$$
N=2 * \tau * f * \frac{F_{\text {burst }}}{F_{\text {releasable }}} * \frac{F_{\text {internalized }}}{F_{\text {releasable }}}
$$

with

- $\quad N$ is the number of release events per synaptic vesicle lifetime.

- $\quad 2$ is a correction factor for the total time a synaptic vesicle remains releasable before its inactivation. This correction factor was necessary since synaptic vesicles can get tagged both during their first or their last release event. On average, this results in synaptic vesicle tagging after half their lifetime as releasable vesicles has passed. To arrive at their total lifetime as part of the releasable population, a multiplication by 2 is necessary. This assumes a Gaussian distribution of release events per vesicle lifetime. 
- $\quad \tau$ is the time constant of a synaptic vesicles staying releasable (obtained from the experiments shown in Figure 3.5C; $\tau=0.5$ days). As indicated in the description of the previous parameter, this underestimates the real time spend by the vesicle in the releasable population by half, which is why the previous factor was introduced.

- $\quad f$ is the frequency of activity bursts occurring in the cultures used here, as measured by $\mathrm{Ca}^{2+}$ imaging. As indicated in Figure 3.10C, $f=0.09 \mathrm{~Hz}$.

- $\frac{F_{\text {burst }}}{F_{\text {releasable }}}$ is the fraction of the releasable population of synaptic vesicles that is released during each neuronal activity burst, as measured by pHluorin imaging. As indicated in Figure 3.10I, $\frac{F_{\text {burst }}}{F_{\text {releasable }}}=0.075$ (release occurring during a single $\mathrm{Ca}^{2+}$ burst divided by release occurring during 600 AP stimulation).

- $\frac{F_{\text {internalized }}}{F_{\text {releasable }}}$ is the fraction of the releasable population that is internalized, and therefore available for release during activity bursts. As indicated in Figure 3.2, $\frac{F_{\text {internalized }}}{F_{\text {releasable }}}=0.45$ (internalized fraction of the releasable population divided by total releasable population, which includes the current surface resident fraction). This is a necessary correction factor to account for the fact that the surface-resident population also participates in release and recycling (see Chapter 1.3 of the Introdcution).

Using this formula, I calculated the number of release events per synaptic vesicle lifetime in rat hippocampal cultures to be $\sim 260$.

\subsection{Ageing synaptic vesicles get contaminated with SNAP25 from the cell membrane}

Thus far, I dissected the life cycle of synaptic vesicles from active participation in release to inactivation at the synapse. I determined that young synaptic vesicles are preferentially released (Figure 3.4; Figure 3.5) and that ageing synaptic vesicles progressively lose their ability to release the more often they are used (Figure 3.9), and that they become inactive after $\sim 260$ rounds of release and recycling (Chapter 3.4; Figure 3.10). But the mechanism of inactivation was still unclear.

\section{Experimental approach to determine changes in synaptic vesicle protein content over} time. The synaptic vesicle comes into contact with several compartments during its cycles of release and recycling (Südhof, 2004) that offer the possibility of making changes in the molecular composition of the vesicle, with potential impact on the release properties of the vesicle. This hypothesis is not in conflict with my earlier claim that synaptic vesicles stay largely intact throughout release and recycling (Chapter 1.4; Chapter 3.3; Chapter 3.4). As 
described earlier, loss of proteins from the vesicle upon fusion and uptake of proteins during recycling are a possibility (Hoopmann et al., 2010). Even small changes in the quantitative composition of vesicles could potentially greatly impact their behaviour (Hua et al., 2011b; Ramirez et al., 2012). There are currently no methods in existence to specifically separate young, releasable synaptic vesicles from old, inactive synaptic vesicles for quantitative biochemical analysis of their respective protein composition. It might in the future become possible to use fluorescence activated vesicle sorting (FAVS), which has been recently derived from FACS (Cao et al., 2008), to do this. However, FAVS currently still is largely diffraction limited and thus not precise enough to separate $40 \mathrm{~nm}$ diameter synaptic vesicles. The current limit for reliable FAVS is $\sim 100 \mathrm{~nm}$ (Momen-Heravi et al., 2012). An alternative would be magnetism activated vesicle sorting (MAVS), but this is not currently feasible either, because the size of the magnetic beads necessary for effective separation, which would have to be delivered into releasable vesicles to tag them, exceeds the diameter of the synaptic vesicle (Belov et al., 2016). To assess changes in the molecular composition of synaptic vesicles over time, I thus used a two-colour STED imaging approach on ultrathin slices. This is not ideal, because it does not allow direct absolute quantifications, as mass spectrometry or Western blotting would (Takamori et al., 2006; Wilhelm et al., 2014). However, the STED approach facilitates the detection of changes in association of proteins of interest with synaptic vesicles at a hitherto unrivalled level of sensitivity and specificity, as it allows the identification of individual synaptic vesicles in non-homogenized synapses.

SNAP25 is increased on old vesicles. To distinguish between young, releasable vesicles and old, inactive vesicles, I performed the co-immunostaining for candidate proteins either directly after antibody live-tagging (day 0 , young vesicles) or 4 days later (day 4 , old vesicles). I selected nine candidate proteins for this approach, based on their abundance (Takamori et al., 2006; Wilhelm et al., 2014) in compartments the synaptic vesicle passes through during its cycle of release and recycling (Südhof, 2004), and based on their significance in synaptic vesicle release (Jahn and Fasshauer, 2012; Rizzoli, 2014). These proteins were: SNAP25 and syntaxin 1 for the cell membrane, VGlut 1/2, VATPase, VAMP2, and synaptotagmin 1 for the synaptic vesicle itself, syntaxin 16 and VAMP4 for the endosome, and synapsin I/II as an abundant cytosolic organiser of the vesicle cluster. There were no detectable significant changes between young and old vesicles for any of these, except for a significant increase, by approximately 87\%, in SNAP25 on old vesicles (Figure 3.11). 


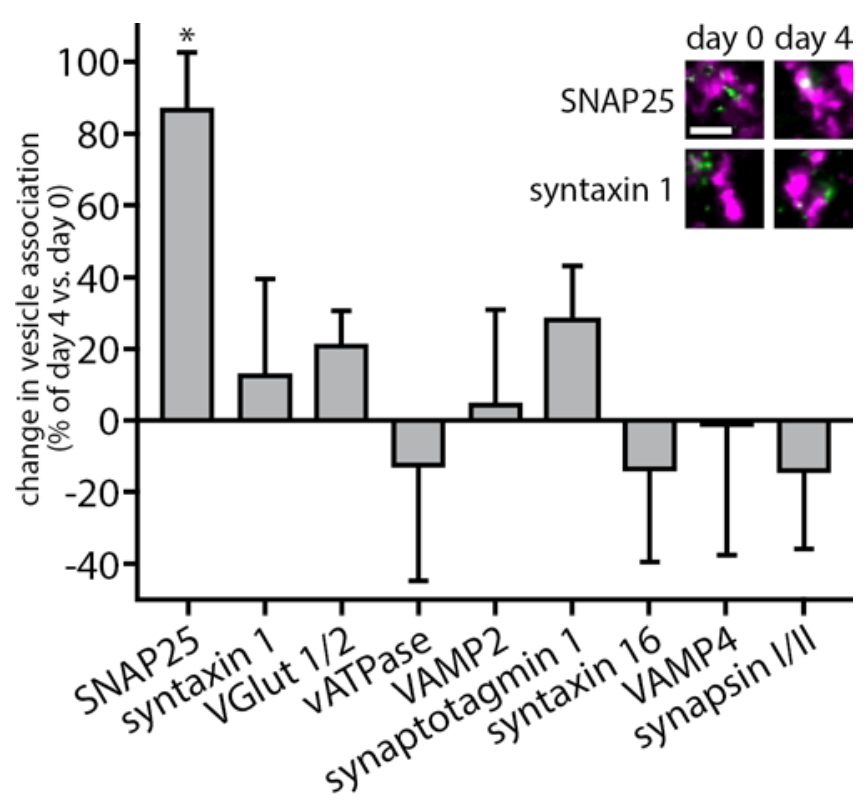

Figure 3.11: Ageing synaptic vesicles get contaminated with SNAP25 from the cell membrane.

Synaptic vesicles were tagged with synaptotagmin 1 antibodies and coimmunostained for a variety of proteins of interest, directly after tagging for young releasable vesicles, or after 4 days for aged inactive vesicles ( $n=4 / 3$ [young, releasable/old, inactive], 3/3, 3/2, 3/4, 4/4, 3/3, $4 / 4,3 / 3,3 / 3$, independent experiments per respective data point; at least 10 neurons sampled per experiment). Two-colour STED imaging of $50 \mathrm{~nm}$ thin sections then revealed

changes in association of the proteins of interest with synaptic vesicles. The only significant change was an increase of association of old vesicles with SNAP 25 by $\sim 87 \%$, compared to young vesicles. Inset: exemplary images of synaptic vesicle (synaptotagmin 1, green) association with proteins of interest (pink), for young, releasable vesicles (day 0) and aged, inactive vesicles (day 4). Scale bar: $500 \mathrm{~nm}$.

All data represent the mean \pm SEM. ${ }^{*} p<0.05$

Vesicular SNAP25 leads to inactivation. To test whether this contamination of ageing vesicles with SNAP25 also has functional implications or is just a bystander effect, I engineered a construct to specifically target SNAP25 to all newly produced synaptic vesicles: sypHy-SNAP25. This consists of the synaptophysin-based pHluorin sypHy C-terminally fused to a version of SNAP25 where the palmitoylation sites for membrane insertion had been removed. I chose sypHy because synaptophysin targets better to synaptic vesicles than most other proteins and because it allows monitoring of vesicle release at the same time. The latter was done by subjecting neurons transfected with either sypHy or sypHy-SNAP25 to a stimulation paradigm designed to release all releasable vesicles (600 action potentials at 20 $\mathrm{Hz}$; Wilhelm et al., 2010). This approach revealed that physiologically normal synapses (sypHy overexpression; Granseth et al., 2006; Li and Murthy, 2001) could release $\sim 40 \%$ of their internalized vesicles, which is comparable to what I measured with antibody live-tagging (Figure 3.2; also see Figure 3.10). Synapses which contain vesicles that are enriched with SNAP25 already during biogenesis, on the other hand, could release less than $10 \%$ of their internalized vesicles during stimulation (Figure 3.12). This significant depression in release indicates that SNAP25 does have a direct effect on the releasability of synaptic vesicles. 


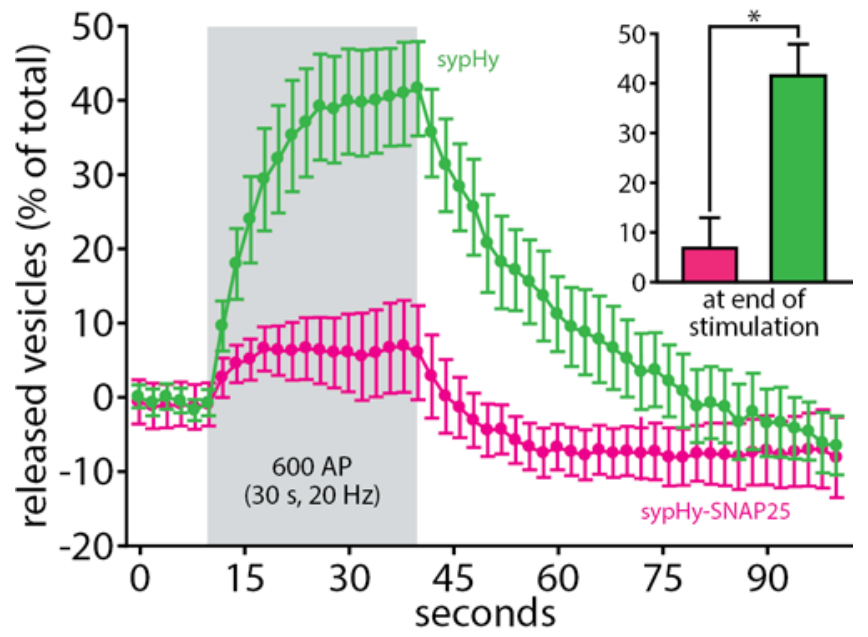

Figure 3.12: SNAP25 on synaptic vesicles results in their inactivation.

Synaptic vesicles carrying a sypHy construct or a sypHy-SNAP25 construct were subjected to stimulation with 600 action potentials (AP) at $20 \mathrm{~Hz}$, and the release was monitored by imaging fluorescence changes of sypHy; the values obtained were then normalized to the total vesicle pool after a pulse of $\mathrm{NH}_{4} \mathrm{Cl}$. Synaptic vesicles carrying SNAP25 through the construct did respond significantly less to

stimulation than their counterparts without direct targeting of SNAP25 $(n=6$ independent experiments for sypHy, $\mathrm{n}=9$ independent experiments for sypHy-SNAP25). Inset: quantification of response at the last time point of stimulation.

All data represent the mean \pm SEM. ${ }^{*} p<0.05$

\subsection{CSPa is a quantitative bottleneck for synaptic vesicle release and} is blocked by vesicular SNAP25 in a molecular timer mechanism

After having determined that ageing synaptic vesicles can be inactivated by contamination with SNAP25 from the cell membrane, I wanted to elucidate the mechanism of this process. How exactly does SNAP25 decrease the releasability of ageing vesicles? To approach this problem, I tried to predict possible interaction partners of SNAP25 on the vesicle that could be blocked in their function in vesicle fusion by interaction with SNAP25 in cis on the vesicle itself rather than in trans with SNAP 25 on the cell membrane. Two proteins in particular qualify as candidates: VAMP2 and CSPa.

VAMP2 as a poor candidate for a quantitative bottleneck. VAMP2 is the most important interaction partner of SNAP25 in the synapse; it is the vesicular SNARE that interacts with the membrane SNAREs SNAP25 and syntaxin 1 to effect vesicle fusion (Blasi et al., 1993a, 1993b; Link et al., 1992; Schiavo et al., 1992; Söllner et al., 1993; Südhof and Rizo, 2011). VAMP2 can further be sequestered into ternary complexes with SNAP25 and syntaxin 1 in cis on the vesicle itself (Otto et al., 1997). VAMP2 is also the most abundant vesicular protein, with $\sim 70$ copies (Takamori et al., 2006; Wilhelm et al., 2014). This very abundance, however, effectively rules it out as a candidate protein that could be blocked by contaminating SNAP25 on the vesicle. Considering that the average synaptic vesicle contains only 1.8 copies of SNAP25 (Takamori et al., 2006), even the increase measured here (Figure 3.11) could not result in enough interactions to block all 70 vesicular copies of VAMP2 in 1:1 interactions (Poirier et al., 1998; Sutton et al., 1998) in cis, especially since two 
copies of VAMP2 seem sufficient for fusion (Sinha et al., 2011). In excess of $90 \%$ of VAMP2 would be left free for fusogenic trans interactions. This is unlikely to drastically inhibit release.

CSPa as an excellent candidate for a quantitative bottleneck. I therefore turned to CSPa as a candidate. CSPa acts in a chaperoning complex with Hsc70 and SGT $\alpha$ (Thakur et al., 2001) that primes SNAP25 on the cell membrane for participation in the SNARE complex (Sharma et al., 2012). There are only 2-3 copies of CSPa per synaptic vesicle, so SNAP25 might well sequester all the CSPa on a synaptic vesicle in non-functional cis-complexes. To test this hypothesis, I performed several overexpression experiments, using wild-type CSPa $\left(\mathrm{CSP}_{\mathrm{WT}}\right)$, a version of CSPa mutated to replace the palmitoylation sites that target it to the vesicle membrane (CSP mut; $_{\text {; }}$ Sharma et al., 2011), and SNAP25 as well as syntaxin 1, as a control, in different combinations (Figure 3.13). I monitored synaptic vesicle release through live-tagging of synaptotagmin 1 with fluorophore-conjugated antibodies.

CSPa as the quantitative bottleneck that sets the molecular timer. If CSPa actually is a quantitative bottleneck for vesicle release and gets blocked after contamination with enough copies of SNAP25, as my hypothesis predicts, overexpression of CSP ${ }_{W T}$ should increase release in synapses. This was indeed the case (Figure 3.13). Almost all synaptic vesicles in synapses overexpressing $\mathrm{CSP}_{\mathrm{WT}}$ were able to release during intrinsic network activity during a $1 \mathrm{~h}$ tagging period (Figure 3.13B). This indicates that the affected synapses can no longer distinguish between young and old vesicles and thus use all of them indiscriminately. $\mathrm{CSP}_{\text {mut, }}$ as expected, did have no effect on release levels (Figure 3.13B), because it could not target to vesicles correctly and thus left them with the same amount of CSPa as without any overexpression. I then overexpressed SNAP25 alone to check if this would reduce release, as did the direct targeting of SNAP25 to vesicles during biogenesis via sypHy-SNAP25 (Figure 3.12). Indeed, this diminished release by $\sim 30 \%$ (Figure 3.13). Next, I tested if overexpression of $\mathrm{CSP}_{\mathrm{WT}}$ could rescue release in neurons also overexpressing SNAP25. This did not only restore the physiological levels of release, but led to the release of almost all vesicles in the synapse, as during overexpression of $\mathrm{CSP}_{\mathrm{WT}}$ alone. This overcompensation hints at a delicate balance between the amount of CSP ${ }_{W T}$ and SNAP25 on the vesicle necessary to control release of ageing synaptic vesicles. As expression levels and the actual copy number of overexpressed $\mathrm{CSP}_{\mathrm{WT}}$ and SNAP25 on the vesicles is practically impossible to control or measure accurately in this system, this issue cannot be dissected further at the moment. As a control, I tested whether overexpression of $\mathrm{CSP}_{\text {mut }}$ together with SNAP25 would have any effect on vesicle release, but as expected, it did not restore release depressed from SNAP25 overexpression. As a further control, I overexpressed syntaxin 1, the other cell membrane SNARE of synaptic release. This was necessary to exclude any off- 
target effects of SNARE overexpression in synapses, since a decrease in release after overexpression of SNAP25 is somewhat unexpected, in the absence of the timer mechanism hypothesis presented here. One would usually expect the overexpression of a SNARE protein to result in an increased release. Nonetheless, a depression of release as an effect of SNAP25 overexpression has been observed before (Owe-Larsson et al., 1999). Also, overexpression of syntaxin 1 leads to an increase in release, comparable to the release induced by overexpression of $\mathrm{CSP}_{\mathrm{WT}}$ (Figure 3.13). This indicates a very specific effect of SNAP25 that supports the timer mechanism hypothesis presented here.
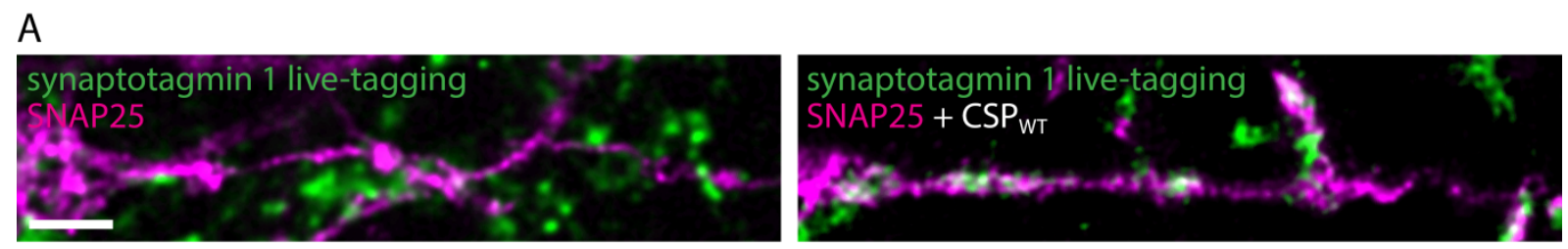

B

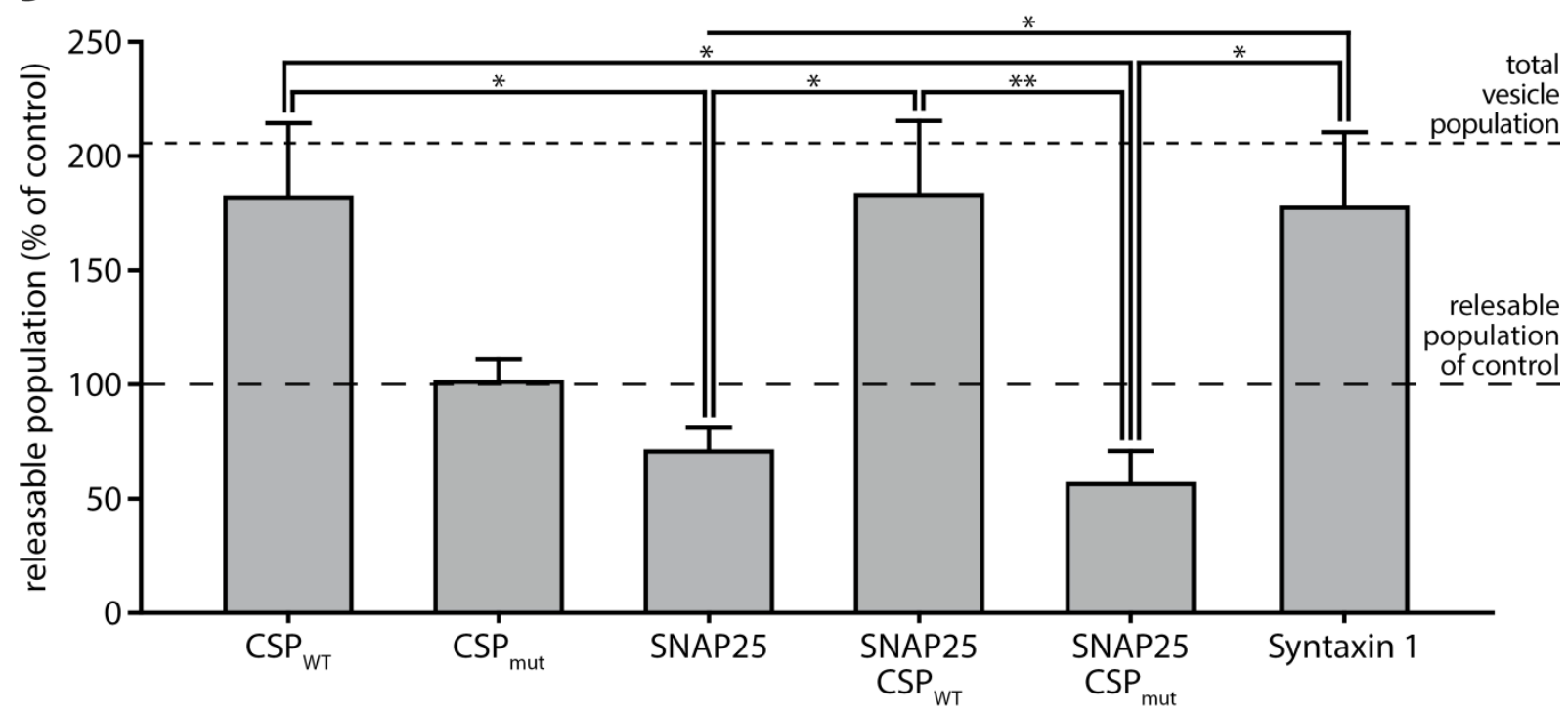

Figure 3.13: CSPa is a quantitative bottleneck for synaptic vesicle release and its interaction with vesicular SNAP25 acts as a timer for vesicle inactivation after $\sim 260$ uses.

(A) Exemplary images of uptake of synaptotagmin 1 antibody as a measure of intrinsic network activity in neurons overexpressing SNAP25 or SNAP25 + wild-type CSPa (CSP $\left.{ }_{\mathrm{WT}}\right)$. Recycling in neurons overexpressing SNAP25 is decreased compared to neurons overexpressing SNAP25 + CSP ${ }_{\mathrm{WT}}$ Scale bar: $2 \mu \mathrm{m}$.

(B) Quantification of release during intrinsic network activity, monitored via uptake of synaptotagmin 1 antibody, in neurons overexpressing $\mathrm{CSP}_{\mathrm{WT}}(\mathrm{n}=17$ transfected neurons from 4 independent experiments), a mutated version of CSPa unable to target to vesicles and thus incapable of interacting with SNAP25 (Sharma et al., 2012; CSP $_{\text {mut; }} \mathrm{n}=11$ transfected neurons from 4 independent experiments), SNAP25 ( $n=24$ transfected neurons from 8 independent experiments), SNAP25 + $\mathrm{CSP}_{\mathrm{WT}}\left(\mathrm{n}=20\right.$ transfected neurons from 8 independent experiments), SNAP25 $+\operatorname{CSP}_{\text {mut }}(n=20$ transfected neurons from 6 independent experiments), and, as a control, syntaxin $1(n=13$ 
transfected neurons from 7 independent experiments). The lower dashed line represents the normal level of activity; the upper dashed line indicates the total vesicle pool at the synapse (see Figure 3.2). All data represent the mean \pm SEM. ${ }^{*} p<0.05,{ }^{\star \star} p<0.01$

A model for the timer mechanism. To summarize these experiments: young synaptic vesicles are made fusion-competent via a chaperoning complex of CSPa, Hsc70, and SGTa that primes cell membrane SNAP25 in a trans-complex (Figure 3.14A; Sharma et al., 2011, 2012; Thakur et al., 2001). The same complex could form in cis on the vesicle itself after contamination with SNAP25 through repeated fusion of ageing vesicles to the cell membrane, which would effectively sequester CSPa from participation in the fusogenic transcomplex, reducing the releasability of the vesicle (Figure 3.14B).

A

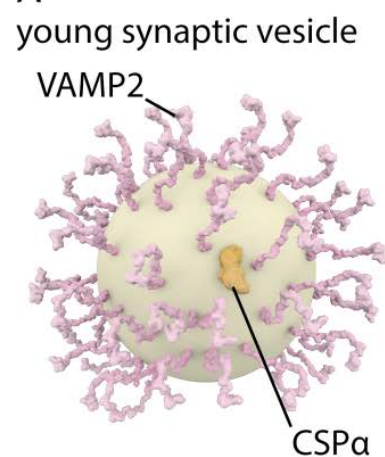

B

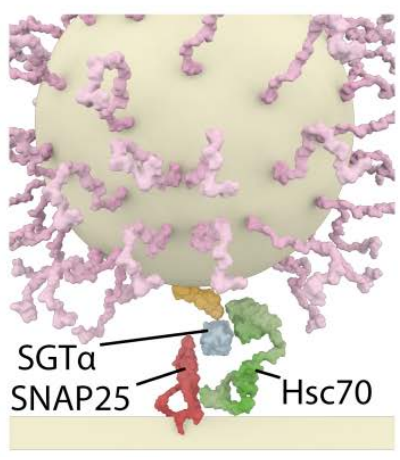
aged synaptic vesicle

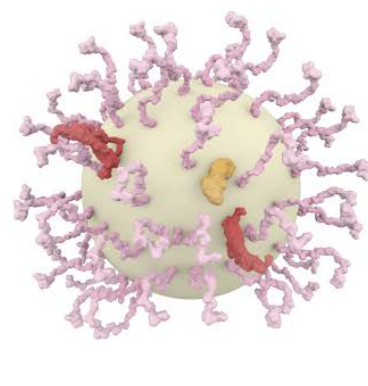

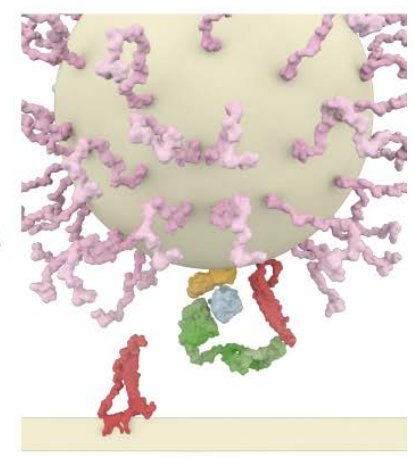

Figure 3.14: A model of the timer mechanism.

(A) CSPa forms a chaperoning complex with Hsc70 and SGTa in trans with SNAP25 on the cell membrane, to prepare the synaptic vesicle for fusion (Sharma et al., 2012).

(B) SNAP25 contamination on aged vesicles could allow the formation of the same complex as in (C), but in cis on the vesicle itself. This non-fusogenic complex would effectively sequester CSPa and prevent it from forming the fusogenic trans-complex.

SNAP25: involvement in vesicle degradation. After having established that contamination with SNAP25 inactivates synaptic vesicles, I finally wanted to investigate whether SNAP25 might also have a role in vesicle degradation. This possibility again emerged from a consideration of protein copy numbers (Wilhelm et al., 2014). It is generally assumed that synaptic vesicle degradation proceeds via fusion to the endolysosomal system (Candiello et al., 2016; Fernandes et al., 2014b; Haberman et al., 2012; Rizzoli, 2014; Uytterhoeven et al., 2011) and autophagy (Binotti et al., 2014; Rizzoli, 2014; Wang et al., 2015). This necessitates the fusion of synaptic vesicles to early endosomes or other precursors of the endolysosomal system. However, there are only about a quarter as many endosomal $\mathrm{Q}_{\mathrm{b}^{-}}$ and $\mathrm{Q}_{c}$-SNARES present at the synapse as there are endosomal $\mathrm{Q}_{a}$-SNAREs (Figure 3.15A; Wilhelm et al., 2014). This might present a bottleneck for the entrance point of synaptic 
vesicles into the degradation pathway; R-SNAREs should not be a bottleneck, as the abundant VAMP2 can act as R-SNARE in endolysosomal fusion (Haberman et al., 2012). I therefore investigated the association of synaptic vesicles with Rab7, a marker of retrogradely transported early endosomes and autophagosomes (Cheng et al., 2015; Deinhardt et al., 2006), during direct targeting of SNAP25 to synaptic vesicles via sypHySNAP25 (as in Figure 3.12), or during overexpression of SNAP25 (as in Figure 3.13). In both cases, there was a marked increase in Rab7 in synaptic compartments (Figure 3.15B-D). This was not due to an increase in synapse size in general, as synaptophysin levels did not change significantly (Figure 3.15D).

A

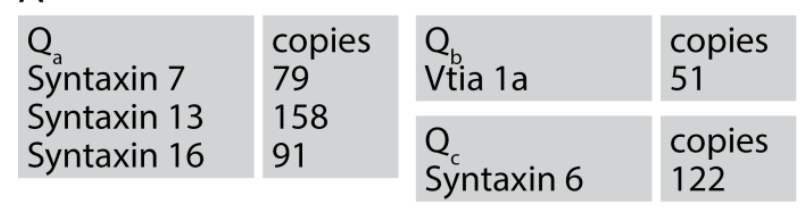

B

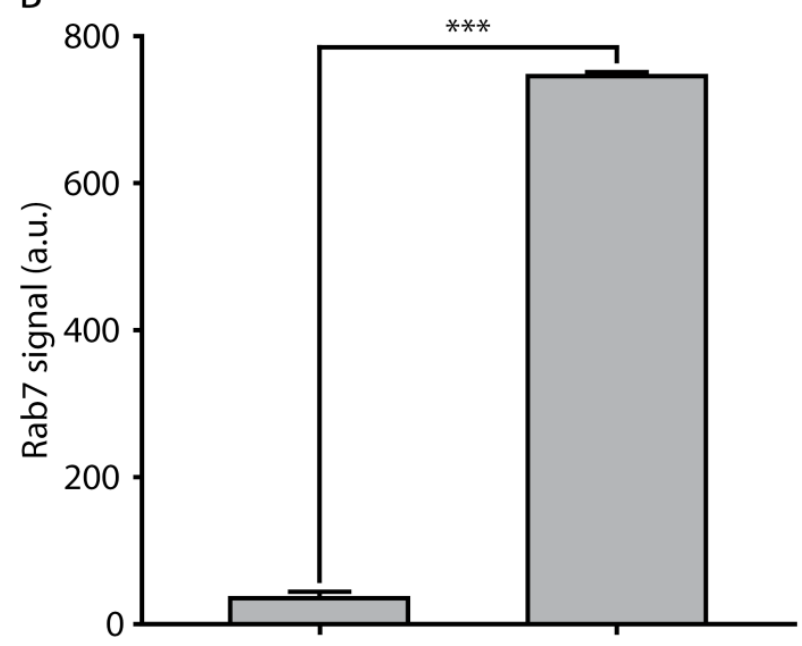

sypHy
C synaptophysin Rab7 control

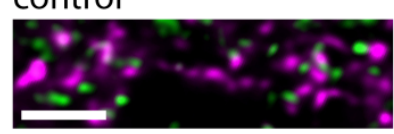

$\mathrm{D}$

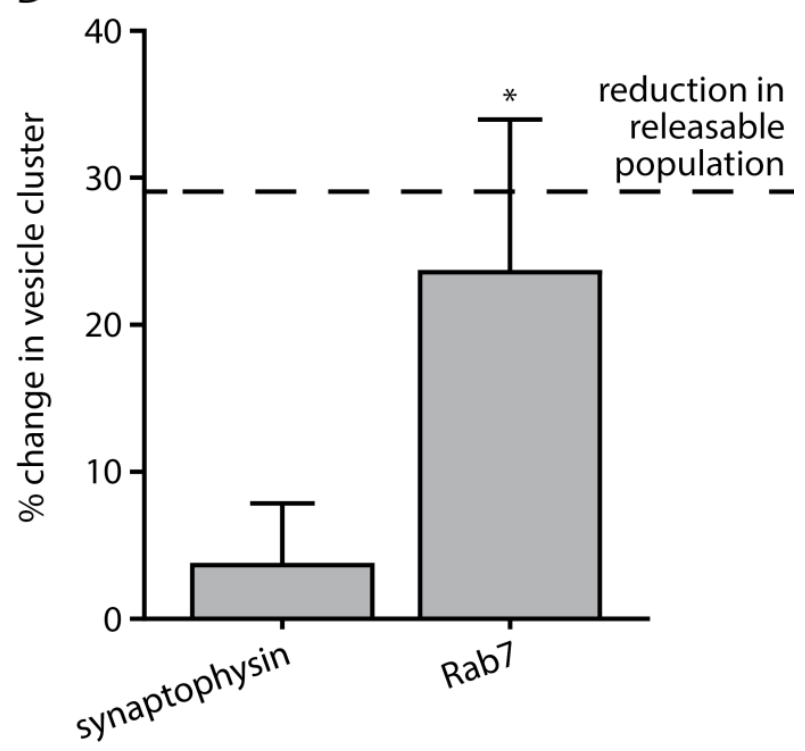

Figure 3.15: SNAP25 contamination of synaptic vesicles coincides with the fusion of synaptic vesicles to early endo-/lysosomal compartments.

(A) A quantitative analysis of endosomal Q-SNAREs in synaptosomes showed that there are many more $\mathrm{Q}_{a}$-SNAREs than $\mathrm{Q}_{\mathrm{b}}$ - and $\mathrm{Q}_{\mathrm{c}}$-SNAREs (from Wilhelm et al., 2014).

(B) Quantification of changes in Rab7 association with vesicle clusters during overexpression of sypHy or sypHy-SNAP25 (as used in Figure 3.12).

(C) Exemplary images of Rab7 in synaptic compartments (identified by synaptophysin) in neurons overexpressing SNAP25 or not overexpressing SNAP25 (control). Scale bar: $2 \mu \mathrm{m}$.

(D) Quantification of changes in Rab7 association with vesicle clusters during overexpression of SNAP25. As a control for effects on synapse size, the changes in synaptophysin levels are shown, too (no significant increase).

All data represent the mean \pm SEM. ${ }^{*} p<0.05,{ }^{* \star *} p<0.005$ 


\subsection{Synaptic vesicle inactivation through a timer for use prevents the use of potentially damaged vesicles}

One remaining open question is why such an elaborate timer mechanism for the inactivation of synaptic vesicles might have evolved. It is important to repeat here that this inactivation precedes degradation by at least $\sim 1$ day, and that a synaptic vesicle spends more time in the inactive state than it spends in the releasable state (Figure 3.5).

Continued use of old vesicles is detrimental to neuronal health and function. First of all, I wanted to check whether the use of aged vesicles is actually detrimental to synapse function or neuronal health. It seems that protracted use of aged vesicles, in conditions where the supply with young vesicles is disrupted, can slightly impair release during highfrequency activity (Figure 3.8D). Furthermore, synapses overexpressing $\mathrm{CSP}_{\mathrm{WT}}$ show an increase in neurite degeneration and synapse swelling that is significantly higher than during overexpression of $\mathrm{CSP}_{\text {mut }}$ (Figure 3.16A,B; overexpression usually always impairs neuron health somewhat, which explains the neurite damage in $\mathrm{CSP}_{\text {mut }}$ neurons). One possible cause for this could be excitotoxicity (Manev et al., 1989), brought about by release of too many vesicles, as old ones are not inactivated properly. In addition, I observed a loss of released vesicle material into the peri-synaptic axonal space during stimulation in conditions where the supply of newly produced vesicles was cut off and only aged vesicles were available (Figure 3.16C,D; see Figure 3.8 for treatments). This is indicative of a problem with endocytosis, possibly through impaired synaptic vesicle integrity or decreased recognition of vesicles by the adapter complexes of clathrin-mediated endocytosis. If an aged vesicle dissociates during release or cannot be recognized properly by the endocytosis machinery, the endocytosis process will necessarily take longer, because the components need to be reclustered first or because their recognition is impaired. This would then stall endocytosis (Neher, 2010) and lead to an accumulation of vesicular material at the peri-active zone (Roos and Kelly, 1999) that might ultimately be lost into the peri-synaptic space or axon. The stalled endocytosis could ultimately also impair release (Neher, 2010), which might explain the neuronal degeneration (Figure 3.16A,B). This all hints at neuronal impairments caused by the use of aged synaptic vesicles, which would explain why they need to be inactivated. 

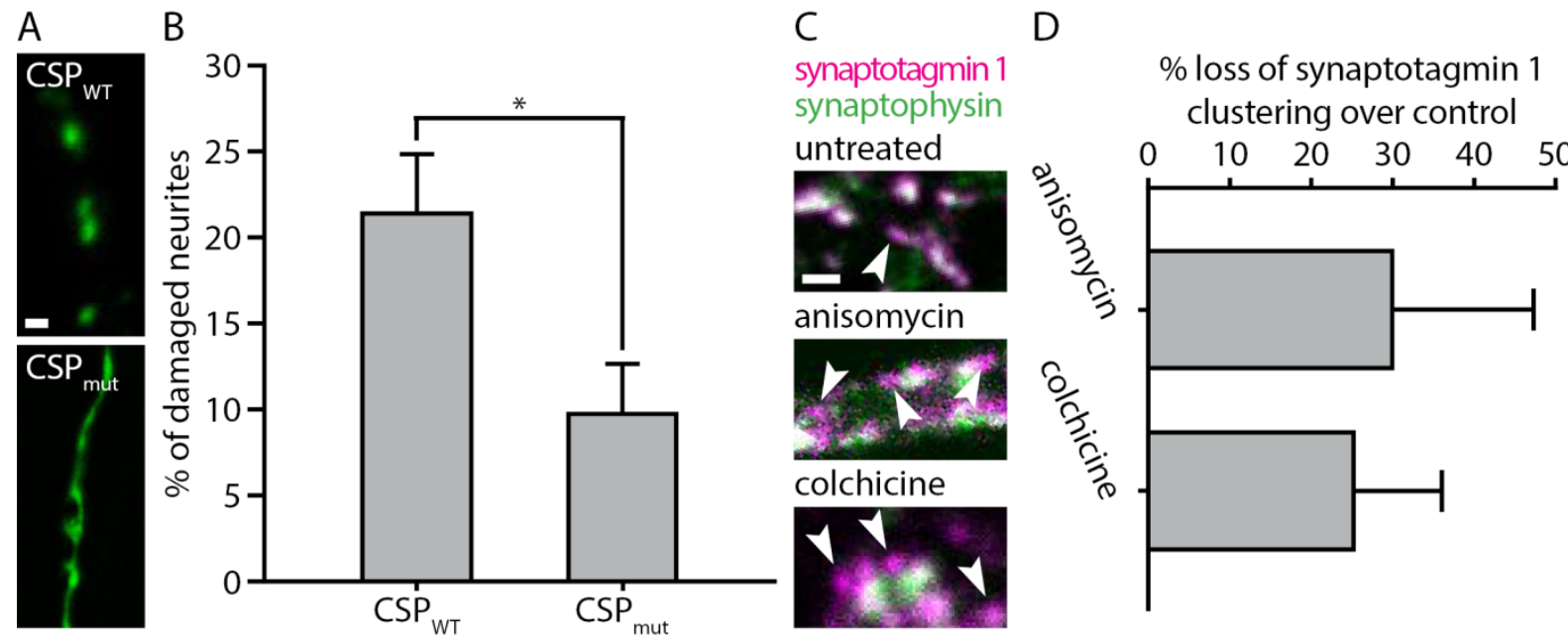

Figure 3.16: Using aged synaptic vesicles leads to neurodegeneration and endocytosis defects.

(A) Exemplary images of neurites from neurons overexpressing $\mathrm{CSP}_{\mathrm{WT}}$ or $\mathrm{CSP}_{\text {mut }}$. As demonstrated in Figure 3.13, the overexpression of $\mathrm{CSP}_{\mathrm{WT}}$ abolishes the timer mechanism of synaptic vesicle inactivation, while overexpression of $\mathrm{CSP}_{\text {mut }}$ leaves it intact. Neurite degeneration in the form of synapse swelling is clearly visible in $\mathrm{CSP}_{\mathrm{WT}}$ overexpressing neurites. The fluorescence shown is not from CSPa, but from co-expressed cytosolic GFP. Scale bar: $1 \mu \mathrm{m}$.

(B) Quantification of neurite damage induced by overexpression of $\mathrm{CSP}_{\mathrm{WT}}$ and $\mathrm{CSP}_{\text {mut. There is some }}$ damage to neurons even from overexpression of $\mathrm{CSP}_{\text {mut }}$, which is usual for any overexpression. The damage induced by overexpression of $\mathrm{CSP}_{\mathrm{WT}}$, however, is significantly higher.

(C) Exemplary images of loss of synaptotagmin 1 from synapses into the peri-synaptic space upon stimulation (600 action potentials, $20 \mathrm{~Hz}$ ). Treatment with anisomycin, to inhibit protein biosynthesis, and colchicine, to disrupt trafficking of newly produced synaptic vesicles from the cell body to the synapse, leads to the protracted use of aged synaptic vesicles (see Figure 3.8). Under these conditions, more live-tagged synaptotagmin 1 is lost from synapses (arrows). Scale bar: $1 \mu \mathrm{m}$.

(D) Quantification of the increased loss of synaptotagmin 1 from the protracted use of aged synaptic vesicles.

All data represent the mean \pm SEM. ${ }^{*} p<0.05$

\section{Synaptic vesicle inactivation is timed to precede accumulation of damage. It is}

puzzling, however, why inactivated synaptic vesicles are not immediately removed by damage response mechanisms. This could be impossible, because of a lack of damage at the time of inactivation. I tried to predict protein damage during the synaptic vesicle life cycle, to compare it with the timing of the stages in the life cycle of the vesicle that I could determine during this Thesis. First of all, this required an estimate of protein damage accumulation on the synaptic vesicle. Data on this issue is practically non-existent, because the question is difficult to address experimentally. There is, however, extensive data on synaptic vesicle protein lifetimes in hippocampal cultures (Cohen et al., 2013). Assuming that protein degradation would only occur after protein damage, as all known degradation mechanisms seem to target either proteins specifically targeted for degradation by direct 
mechanisms or damaged proteins (Goldberg, 2003; Rubinsztein, 2006), I could use this data to predict accumulation of protein damage. To do that, I first weighted the average lifetimes (Cohen et al., 2013) of all published major synaptic vesicle proteins by their copy numbers (Wilhelm et al., 2014), to arrive at the average lifetime of the synaptic vesicle, with standard deviation ( $3.8 \pm 0.8$. days, mean $\pm \mathrm{SD}$; Figure 3.17A). This can be used to plot a cumulative Gaussian, or probability density function, of synaptic vesicle degradation, which under the above assumption equates to synaptic vesicle protein damage (Figure 3.17B). So how much damage would a synaptic vesicle have accumulated at the time it gets inactivated? Assuming $\sim 12 \mathrm{~h}$ for biogenesis, transport, and maturation of a newly synthesized synaptic vesicle precursor until it enters the releasable population (Karra et al., 2010; personal observation from overexpression of GFP-tagged synaptic vesicle proteins; also see Legend of Figure 3.17), the inactivation of synaptic vesicles (derived from Figure 3.5C) comes at a time when the vesicle has accumulated very little damage ( $-0.2 \%$ of all vesicle proteins, or less than one protein copy, on average; Figure 3.17B). After inactivation, however, damage rapidly increases to the inflection point (50\% damaged proteins) at 3.8 days (Figure $3.17 \mathrm{~B}$ ), which coincides almost perfectly with the lifetime of synaptic vesicles experimentally determined in this Thesis (derived from Figure 3.4A,B; see Legend of Figure 3.17 for further elaboration). Extending the releasable period of the synaptic vesicle by $12 \mathrm{~h}$ would result in damage to an average of $-1.4 \%$ of all vesicle proteins, or one protein copy; extending the releasable period by $24 \mathrm{~h}$ would already result in damage to an average of $\sim 6 \%$ of all vesicle proteins, or at least four protein copies. This indicates that synaptic vesicles are indeed removed from synaptic release before they have a chance to accumulate damage, and with a margin of error of about one lifetime in the releasable population. This is presumably necessary to avoid the use of synaptic vesicles that are even slightly damaged, so that the sensitive process of neurotransmission is not disrupted in any way (see Discussion for further elaboration, especially Chapter 4.5). 
A

\begin{tabular}{l|l|l|}
\hline protein & copy number & lifetime \\
\hline synaptophysin & 31 & 4.1 days \\
\hline syntaxin 1 & 6 & 5.4 days \\
\hline SNAP25 & 2 & 2.3 days \\
\hline synaptotagmin 1 & 15 & 2.7 days \\
\hline synaptogyrin 1 & 2 & 5.2 days \\
\hline SV2a & $10(\mathrm{SV} 2 \mathrm{a} / \mathrm{b})$ & 3.8 days \\
\hline SV2b & $10(\mathrm{SV} 2 \mathrm{a} / \mathrm{b})$ & 3.4 days \\
\hline VATPase a1 & 1 & 3.6 days \\
\hline total & 67 & $3.8 \pm 0.8$ days \\
\hline
\end{tabular}

B

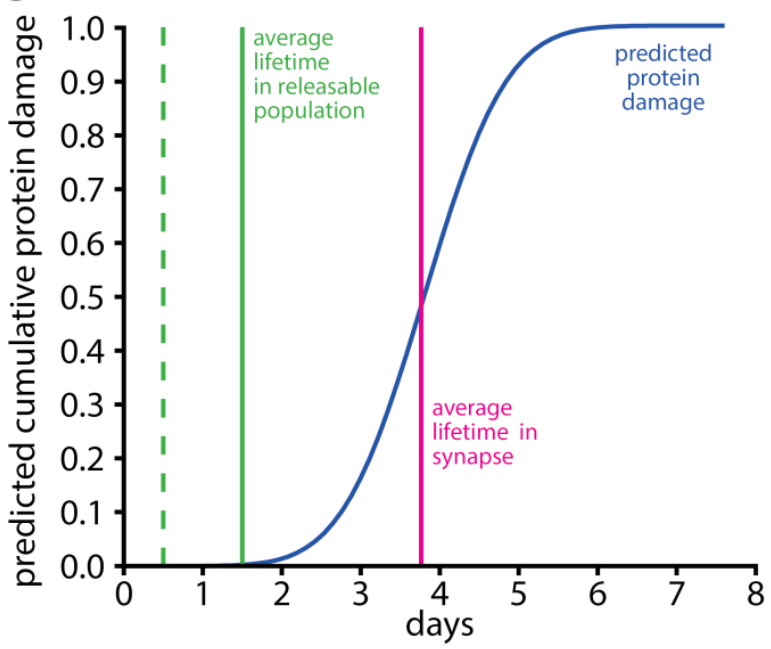

Figure 3.17: Synaptic vesicle inactivation via the timer mechanism pre-empts damage.

(A) Protein copy numbers (from Wilhelm et al., 2014) and protein lifetimes in hippocampal cultures (from Cohen et al., 2013), for the major synaptic vesicle proteins. The average lifetime (mean \pm SD) of the synaptic vesicle (in the row "total") was calculated by weighting the individual protein lifetimes by their copy number.

(B) A cumulative Gaussian distribution, or probability density function, of predicted protein damage. This assumes that proteins are only degraded after they have been damaged. With this assumption, the mean and SD from (A) can be used to predict accumulation of damage, as shown in the plot. The dashed green line indicates the approximate time at which synaptic vesicles produced in the cell body reach the synapse (based on experience with overexpression of GFP-tagged synaptic proteins). The solid green line indicates the time point at which synaptic vesicles turn from the releasable state to the inactive state $(\tau=0.5$ days, derived from Figure 3.5C, times 2 plus the time it takes to reach the synapse; see Chapter 2.13; see Chapter 2.14). The pink line indicates the average lifetime of synaptic vesicles in the synapse ( $T=1.6$ days, derived from Figure 3.4A,B, times 2 plus the time it takes to reach the synapse; see Chapter 2.13; see Chapter 2.14). The vesicles thus become inactive just as the predicted accumulation of damage starts. The experimentally determined average lifetime of synaptic vesicles (pink line) intersects the predicted damage accumulation almost precisely at the $50 \%$ value, so at the peak of the Gaussian distribution used to plot this probability density function, or the average value of the population. 


\section{Discussion}

\subsection{The synaptic vesicle life cycle revisited}

In the Introduction, I proposed a somewhat preliminary synaptic vesicle life cycle, synthesized from the published data (Chapter 1.5; Figure 1.5). This life cycle contained several uncertainties and conjectures, particularly regarding the transitions of vesicles from the releasable to the inactive state (also see Chapter 1.4). For example, it was unclear whether synaptic vesicle activity is linked to certain stages in the vesicle life cycle, whether synaptic vesicles switch frequently between the releasable state and the inactive state, and when and how inactivation and degradation do occur. From the data presented in this Thesis, I can now update the synaptic vesicle life cycle regarding these aspects (Figure 4.1).

Permanent inactivation of ageing synaptic vesicles. Ageing synaptic vesicles are less releasable than young synaptic vesicles (Figure 3.4; Figure 3.5; Figure 3.6). As the vesicles age, they participate less in evoked release, both from intrinsic network activity (Figure 3.5; Figure 3.6) and from external stimulation (Figure 3.4). I observed this behaviour with two independent approaches, synaptic vesicle protein tagging with lumenal antibodies (Figure 3.4; Figure 3.5) and CLICK chemistry tagging of novel genetic constructs (Figure 3.6), and for three different proteins, synaptotagmin 1 (Figure 3.4; Figure 4.5), VGAT (Figure 3.4), and VAMP2 (Figure 3.6). This is supported by earlier observations that synaptic vesicles labelled with FM-dyes in frog neuromuscular junctions cannot be induced to unload the dye through evoked release after a resting period of a few hours (Richards et al., 2000, 2003; Rizzoli and Betz, 2004). Another study, employing super-resolution STED microscopy to follow individual vesicles, found that synaptic vesicles tagged with lumenal synaptotagmin 1 antibodies during release and recycling start out quite mobile, but become relatively immobile after a few minutes (Kamin et al., 2010). This is in line with the idea that inactive synaptic vesicles are immobilized by the synapsin/cytoskeleton meshwork (see Chapter 1.4). These studies, however, had to short a timeframe to confirm that the inactivated synaptic vesicles could not become releasable again after a while. I remedied this with my approaches, which allow functional investigations of tagged synaptic vesicles for up to 10 days. This timescale spans the entire life cycle of the synaptic vesicle, which is on average 2-4 days long in hippocampal cultures (Cohen et al., 2013; Daly and Ziff, 1997).

Dependence on young vesicles. The synaptic vesicles that are releasable are not just younger than their inactive counterparts, they seem to be the youngest vesicles present in the synapse (Figure 3.7). I could reveal this by metabolic tagging of releasing synaptic vesicles, and detection of the tag in super-resolution microscopy. Again, I used two different 
approaches: FUNCAT (Dieterich et al., 2011) and COIN (Saka et al., 2014b). Both techniques showed that releasable synaptic vesicles are young. I could further show, by inhibiting the re-supply of the synapse with newly produced vesicles, that young synaptic vesicles are necessary to replace the releasable population and that lack of replenishment with young vesicles ultimately results in defects in endocytosis and neuronal health (Figure 3.16). This distribution of labour - release of young vesicles, inactivity of old vesicles - is not mere chance. A possible evolutionary explanation for the inactivation of ageing synaptic vesicles will be presented below (Chapter 4.5).

Contamination of ageing synaptic vesicles with SNAP25. As young, releasable synaptic vesicles repeatedly fuse with the cell membrane, which they do $\sim 260$ times during their lifetime in hippocampal culture (Chapter 3.4), they have a chance to become contaminated with proteins from the cell membrane or endosomes, or to lose synaptic vesicle proteins to these compartments (Hoopmann et al., 2010; Truckenbrodt and Rizzoli, 2014). I investigated this question with super-resolution STED microscopy on ultrathin sections. I found only one significant change: SNAP25 is increased on old, inactive synaptic vesicles (Figure 3.11). This change raises the number of SNAP25 copies to an approximately 1:1 ratio with CSPa (see Chapter 4.2 for an in-depth treatment of this estimate). This is an event specific to SNAP25, as syntaxin 1 did not get taken up (Figure 3.11). The most likely explanation for this difference is that there is a much steeper concentration gradient between cell membrane for SNAP25 than for syntaxin 1, and that the membrane insertion via palmitoylation of SNAP25 might favour integration into the vesicle membrane (see Chapter 4.3 for an in-depth treatment of this aspect).

\section{Inactivation of aged synaptic vesicles via a CSPa bottleneck closed by SNAP25. In} Chapter 3.6 of the Results section I introduced the possibility (Figure 3.12) that CSPa might get sequestered in non-fusogenic trans-complexes with SNAP25 on the vesicle membrane (see Chapter 4.3 for in-depth discussion of the timer mechanism), which prevents it from participating in the fusogenic cis-complex with SNAP25 on the cell membrane that is necessary to prime SNAP25 for fusion (Sharma et al., 2011, 2012). CSPa is a palmitoylated protein of the synaptic vesicle membrane that forms a trimeric chaperoning complex with the soluble Hsc70 and SGTa that primes SNAP25 on the cell membrane for participation in the SNARE complex with syntaxin 1 and VAMP2 that facilitates synaptic vesicle release (Evans et al., 2003; Sharma et al., 2011; Thakur et al., 2001). The same complex that usually forms in trans between vesicle and cell membrane (Figure 3.14A) to prime vesicles could also form in cis on the vesicle itself (Figure 3.14B). Sequestering just one of the 2.4 to 2.8 copies of CSPa (Takamori et al., 2006; Wilhelm et al., 2014) might not be enough to seriously reduce 
the releasability of the vesicle, but sequestering two might already suffice. This is evidenced by a severe reduction in release upon overexpression of a construct that specifically targets SNAP25 to synaptic vesicles (Figure 3.12) as well as moderate reduction in release upon overexpression of wild-type SNAP25 (Figure 3.13).

Degradation. Synaptic vesicles are degraded in acidic compartments after anterograde transport to the cell body (Figure 3.3). SNAP25 could be involved in the fusion of synaptic vesicles to endolysosomal organelles (Sun et al., 2003). The necessity of contamination of synaptic vesicles with the $\mathrm{Q}_{\mathrm{bc}}$-SNARE SNAP25 for entering the degradation pathway is made particularly likely because of a marked absence of endosomal $\mathrm{Q}_{\mathrm{bc}}$-SNAREs at the synapse, while endosomal $\mathrm{Q}_{a}$-SNAREs are much more prevalent (Figure 3.15A) and VAMP2 can act as R-SNARE. I determined that Rab7, a marker for organelles of the early endolysosomal degradation pathway, is more prevalent in synaptic compartments upon overexpression of SNAP25 or sypHy-SNAP25 (Figure 3.15). This points to a direct involvement of SNAP25 in synaptic vesicle degradation (see Chapter 4.4 for further details). 


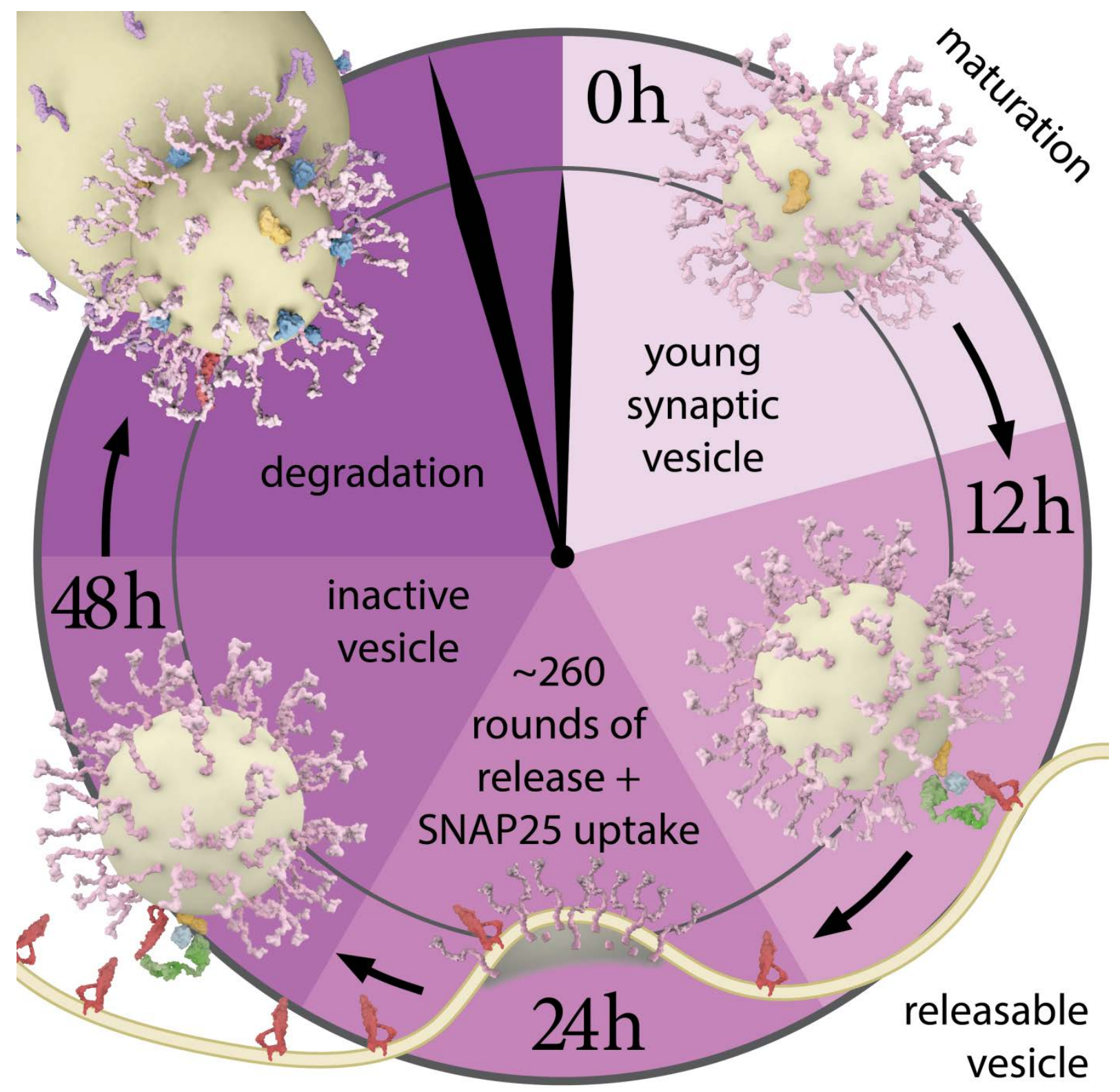

Figure 4.1: The synaptic vesicle life cycle revisited.

Synaptic vesicles become releasable after they finish maturation, which takes an estimated $\sim 12 \mathrm{~h}$ (for clarity, only VAMP2 is shown as a major vesicle component, in pink). Young synaptic vesicles are able to respond to stimulation with ease and participate in release for $\sim 12-24 \mathrm{~h}$. During this time, they are fused to the cell membrane and retrieved from it approximately 260 times in hippocampal culture. Release itself is mediated by a chaperoning complex consisting of the vesicle-bound CSPa (yellow) and the soluble co-factors Hsc70 (green) and SGT (light blue), which act together to prime SNAP25 (red) on the cell membrane for fusion, in a trans-complex. During the repeated fusion events, synaptic vesicles pick up SNAP25 from the cell membrane. This leads to a sequestration of CSPa by SNAP25 in cis-complexes on the vesicle itself. CSPa is thus unavailable to participate in the fusogenic transcomplex. This inactivates the vesicle; an irreversible change of releasability. After another $24-48 \mathrm{~h}$ in the synapse, the synaptic vesicle is fused to endolysosomal compartments, identified by Rab7 (dark blue), presumably through direct interaction of vesicular SNAP25 and endosomal SNAREs (violet). 


\subsection{Timing and quantification of the synaptic vesicle life cycle}

The synaptic vesicle life cycle has previously been mostly described in qualitative terms. From the data gathered in this Thesis, and combined with other published data, I could derive several quantitative parameters, particularly regarding the timing of events in the synaptic vesicle life cycle. It should be noted that all these quantifications are derived rather than measured directly, because the quantified parameters themselves cannot currently be measured directly. Furthermore, the parameters presented here are most likely specific to hippocampal cultures. However, since most of these parameters are most likely linked and therefore likely scale with each other, these quantifications may nonetheless serve as a tentative experimental framework also for studies of other preparations.

Synaptic vesicle life time in culture: 3-4 days. The lifetime of different synaptic vesicle proteins has been determined before, and was found to vary between 2-4 days in culture (Cohen et al., 2013; Daly and Ziff, 1997); this is probably at least 4- to 5-fold longer in situ (Price et al., 2010; Rosenberg et al., 2014; Eugenio F. Fornasiero, personal communication). A systematic analysis performed in this Thesis of the comprehensive study by Cohen et al. (2013) combined with information on the copy numbers of the synaptic vesicle proteins (Takamori et al., 2006; Wilhelm et al., 2014) yielded an average lifetime of the synaptic vesicle of $3.8 \pm 0.1$ days (mean \pm SEM; Figure 3.17). Note the low error, which is probably well within the measurement error; this indicates that synaptic vesicle proteins largely age together (see Chapter 1.2). These numbers could be further confirmed experimentally in this Thesis, based on antibody-tagging of synaptotagmin 1 and VGAT (Figure 3.4A,B; Figure 3.17). This total lifetime of the synaptic vesicle presents a first indispensable step towards the quantitative long-term temporal dynamics of this organelle.

\section{Time spent as releasable vesicle before inactivation: 12-24 $h$. As discussed above} (Chapter 4.1), synaptic vesicles start their life in the synapse as releasable synaptic vesicles and then become progressively less releasable (Figure 3.4; Figure 3.5; Figure 3.6; Figure 3.7). Using a dual-tagging approach with antibodies in living cells, I could further assert that tagged synaptic vesicles are inactivated with a time constant of 0.5 days (Figure 3.5). The tagged vesicles are necessarily a mixed population of very young vesicles that just performed their first release and aged vesicles that are just about to become inactive, and anything in between. Accordingly, the tagged population will, on average, already have spent half of its life in the releasable population, or already have undergone $\sim 130$ release events. To arrive at the total time spent by a synaptic vesicle in the releasable population, we thus have to multiply the measured time constant by two (also see Chapter 2.13 in Materials \& 
Methods), which yields the time spent in the releasable population of each vesicle as approximately one full day.

Number of synaptic vesicle release events per lifetime: 260. The number of release events a synaptic vesicle can undergo is a key parameter to understanding its basic physiology. Changes in the number of release events a vesicle can undergo might ultimately also link to certain neurodegenerative diseases. There have, however, until now not even been estimates for how often a synaptic vesicle can be released. After determining the time a vesicle spends in the releasable population (Figure 3.5), the activity burst frequency in hippocampal cultures (Figure 3.10C), and the release per activity burst (Figure 2.10I), I could calculate the number of release events undergone by synaptic vesicles during their lifetime: -260 (Chapter 3.4). This is, to my knowledge, not only the first time that the number of usages has been quantified for the synaptic vesicle, but the first such estimate for any organelle. It is difficult to precisely predict how the overexpression of sypHy-SNAP25 (Figure 3.12) or wild-type SNAP25 (Figure 3.13) impacts the number of release events a vesicle can go through before inactivation. As the principle of inactivation of ageing vesicles seems to be that they are simply out-competed by young vesicles (see Chapter 4.3 for an in-depth treatment of this issue), the reduction in release that is evident in these two conditions most likely simply reflects a faster inactivation of young vesicles. This assumes that the neurons cannot increase the frequency of synaptic vesicle biogenesis (see below) significantly during the timeframe of the overexpression experiment (typically 3-4 days, see Chapter 2.9 of Materials and Methods). The faster inactivation occurs because the synaptic vesicles have a higher chance to take up SNAP25, when the number of SNAP25 that is present during biogenesis (sypHy-SNAP25) or on the plasma membrane (wild-type SNAP25) is increased. Assuming that the reduction in release under both conditions ( $-30 \%$ for wild-type SNAP 25 , $\sim 85 \%$ for sypHy-SNAP25; Figure 3.12; Figure 3.13) directly corresponds to the reduction in release events, we can derive that synaptic vesicles in neurons overexpressing wild-type SNAP25 are inactivated after $\sim 180$ rounds of release and that synaptic vesicles overexpressing sypHy-SNAP25 average only $\sim 50$ rounds of release.

Frequency of synaptic vesicle biogenesis: one vesicle every $3.3 \mathrm{~s}$. From hippocampal cultures with a seeding density identical to the ones used in this Thesis, we know that there are $\sim 200$ synapses per neuron (Cullen et al., 2010). Each of them contains, on average, -194 internalized synaptic vesicles (Schikorski and Stevens, 1997). Of these, 58 qualify as releasable (Figure 3.2). Of these, the synapse will release, on average, $\sim 4.3$ per synaptic activity burst (Figure 3.10I), which come with a frequency of $0.09 \mathrm{~Hz}$ (Figure 3.10C). That means that the entire neuron releases, on average, $\sim 78$ vesicles per second. If the amount of 
releasable synaptic vesicles is to be maintained constant, this also means that the neuron needs to produce the equivalent of these 78 expended release events in newly produced synaptic vesicles to compensate, during the same time. As the number of release events a synaptic vesicle is good for is now known ( 260 release events, see above), the rate of synaptic vesicle biogenesis in cultured hippocampal neurons can be approximated:

$$
R=\frac{N}{N_{S V} * \frac{F_{\text {internalized }}}{F_{\text {internalized }}+F_{\text {Spontaneous }}+F_{\text {inactive }}} * \frac{F_{\text {burst }}}{F_{\text {releasable }}} * f * N_{\text {synapses }}}
$$

with

- $\quad R$ is the rate of biogenesis of synaptic vesicles in cultured hippocampal neurons, i.e. how long it takes, on average, for one new vesicle to be assembled per neuron.

- $\quad N$ is the number of release events per synaptic vesicle lifetime.

- $\quad N_{S V}$ is the number of synaptic vesicles per synapse in hippocampal culture. $N_{S V}$ is -194 (Schikorski and Stevens, 1997).

- $\frac{F_{\text {internalized }}}{F_{\text {internalized }}+F_{\text {spontaneous }}+F_{\text {inactive }}}$ is the releasable fraction of all internalized synaptic vesicles in hippocampal culture. Derived from Figure 3.2 , this is $\sim 0.30$ (internalized fraction of the releasable population divided by total internalized population).

- $\frac{F_{\text {burst }}}{F_{\text {releasable }}}$ is the fraction of the releasable population of synaptic vesicles that is released during each neuronal activity burst, as measured by pHluorin imaging. As indicated in Figure 3.10l, $\frac{F_{\text {burst }}}{F_{\text {releasable }}}=0.075$ (release occurring during a single $\mathrm{Ca}^{2+}$ burst divided by release occurring during 600 AP stimulation).

- $\quad f$ is the frequency of activity bursts occurring in the cultures used here, as measured by $\mathrm{Ca}^{2+}$ imaging. As indicated in Figure 3.10C, $f=0.09 \mathrm{~Hz}$.

- $\quad N_{\text {synapses }}$ is the number of synapses per cultured hippocampal neuron. For neurons with a seeding density identical to that of the cultures used in this Thesis, $N_{\text {synapses }}$ is $\sim 200$ (Cullen et al., 2010). Another estimates from autaptic cultures is $\sim 277$ (Ikeda and Bekkers, 2008), but this is most likely an artefact, mimicked by lower seeding density in non-autaptic cultures (Cullen et al., 2010).

From this, the rate of synaptic vesicle production can be calculated to be $\sim 0.30 \mathrm{~Hz}$, or one newly produced synaptic vesicle every $3.3 \mathrm{~s}$. Note that this formula essentially calculates how long it takes to expend a number of release events equal to the number of release events one synaptic vesicle can undergo during its lifetime, which is $\sim 260$, as determined from Equation (1). To maintain the releasable population of synaptic vesicles across all 
synapses, the neuron needs to replace the expended release potential by production of a new vesicle in the same time frame, at least on average.

Validity of the compound parameters. Are the numbers presented thus far in line with the release frequency and the number of synaptic vesicles in the synapse, i.e. can the synapse maintain release with these parameters? Or in other words: are these quantifications realistic? An average synapse in hippocampal cultures contains $\sim 194$ internalized synaptic vesicles (Schikorski and Stevens, 1997), of which $\sim 58$ will be releasable (Figure 3.2; also see above). Adding the surface population to that yields a total of $\sim 129$ releasable vesicles per synapse (derived from Figure 3.2). These will, on average, each release 260 times over a period of $24 \mathrm{~h}$. That means that a synapse can support an average of 33,523 release events per $24 \mathrm{~h}$. How many release events will actually occur during that time? The synapse triggers one activity burst every $\sim 11 \mathrm{~s}(0.09 \mathrm{~Hz}$ frequency; see Figure $3.10 \mathrm{C})$, with 4.3 vesicles, on average, being released per activity burst (derived from Figure 3.2, Figure 3.10I, and Schikorski and Stevens, 1997; see above), resulting in a total of 33,437 release events per $24 \mathrm{~h}$. This matches remarkably well to the predicted release events supported by the neuron during the same amount of time, lending additional credibility to these quantifications. To assume 260 release events over a period of $24 \mathrm{~h}$ for all releasable vesicles present at the synapse is not an overestimation, as new vesicles entering the synapse will compensate for old vesicles lost from the releasable population. Is this turnover actually sufficient to replenish the releasable population? During $24 \mathrm{~h}$, each synapse will receive $\sim 131$ newly produced vesicles (easily calculated from the previous section), accounting for a complete turnover of the releasable population ( 129 , see above) during a period of $24 \mathrm{~h}$. This fits very well with the total time each vesicle spends in the releasable population, which is $\sim 24 \mathrm{~h}$ (see above). These verifications lend added confidence to the assumption that the quantifications derived here are accurate enough to serve as a framework for timing in the synaptic vesicle life cycle.

\section{Number of SNAP25 copies on old and on young vesicles: 0.97 and 1.94 respectively.}

The average synaptic vesicle contains 1.8 copies of SNAP25 (Takamori et al., 2006), but this number was derived from a mixed population of young, releasable vesicles and old, inactive vesicles. Assuming that the sample of vesicles in the preparation used for quantification by Takamori et al. (2006), which was homogenized rat cortices, is representative, i.e. that the vesicle isolation process did not favour young or old vesicles, this means that approximately $13.6 \%$ of these vesicles were young and releasable (Marra et al., 2012). As determined in this Thesis, SNAP25 levels are $\sim 87 \%$ higher on old vesicles than on young vesicles (Figure 3.11). Taken together, we can thus derive an average of 0.97 copies of SNAP25 per young vesicle (Chapter 2.13; Equation (3)), and an average of 1.94 copies of SNAP25 per old 
vesicle (Chapter 2.13; Equation (4)). This emphasizes how critical even apparently minor changes in protein copy numbers can be (Gordon et al., 2016). In the model, put forward in this Thesis, of a quantitative CSPa bottleneck that is stoppered by SNAP25 (Chapter 3.7; Chapter 4.3; Figure 3.13), the releasability of a synaptic vesicle is ultimately determined by the quantitative balance between CSPa and SNAP25. Raising the average number of SNAP25 copies from 0.97 to 1.94 seems to be enough to diminish the release probability so drastically that young vesicles (0.97 copies of SNAP25) outperform old vesicles (1.94 copies of SNAP25) by at least a factor of 3 (time point 4 days in Figure 3.5 corresponds to the time point at which the protein association with old vesicles was measured). It is difficult to precisely predict how many more copies of SNAP25 would be present in ageing synaptic vesicles of neurons overexpressing sypHy-SNAP25 or wild-type SNAP25. Since the main determinant of release of ageing synaptic vesicles likely is competition for release with young synaptic vesicles (see Chapter 4.3), the number of SNAP25 on inactivated vesicles in neurons overexpressing wild-type SNAP25 might not be increased at all. The reduction in release would then merely reflect the inability of the neuron to upregulate synaptic vesicle production (and turnover, as synapse size stays constant; Figure 3.15D) to compensate for the earlier loss of vesicles from the releasable population, and not an increase of SNAP25 on aged vesicles per se. These vesicles would simply reach that number sooner. If production is not increased, that would simply reduce the size of the releasable population, like drawing more water from a basin without also increasing the influx of water. The case is different for overexpression of sypHy-SNAP25, as the synaptophysin component of the construct would presumably target SNAP25 to the vesicles already during biogenesis, which would indeed increase the number of SNAP25 on the vesicle, but not just on the ageing vesicle. Considering that the synaptic vesicle is a densely packed structure (Takamori et al., 2006), it cannot accommodate a vast number of overexpressed proteins. Studies of pHluorin-tagged synaptophysin, VGlut, and VAMP2 suggest that a maximum of $\sim 2-3$ copies of overexpressed protein are targeted per vesicle, 3.1 for synaptophysin specifically (Sinha, 2011). This would, however, already put approximately twice as many copies of SNAP25 (3.1 overexpressed copies plus 0.97 copies of endogenous SNAP25 from biogenesis, see above) on a young vesicle as are present on an aged vesicle under physiological conditions (1.94 copies, see above). These $\sim 4.1$ copies of SNAP25 (3.1 overexpressed copies plus 0.97 copies of endogenous SNAP25 from biogenesis, see above) could already be enough to sequester all 2.4 to 2.8 copies of CSPa (Takamori et al., 2006; Wilhelm et al., 2014) (semi-)permanently. This would explain the severe $-85 \%$ reduction in release observed during overexpression of sypHy-SNAP25 (Figure 3.12). From these considerations, we can tentatively predict the relationship of SNAP25-level on the vesicle and releasability (Figure 4.2A), which best fits a 3-parameter hyperbolic decay. Incidentally, this is exactly the type of relationship one would 
expect if the releasability of synaptic vesicles indeed depends on a reversible cis-interaction of SNAP25 and CSPa (see Chapter 4.3 for more details). Taking into account the ratio of SNAP25 to CSPa on the vesicle, which is ultimately probably the better predictor of releasability, the same relationship towards releasability can be observed (Figure 4.2B).

A

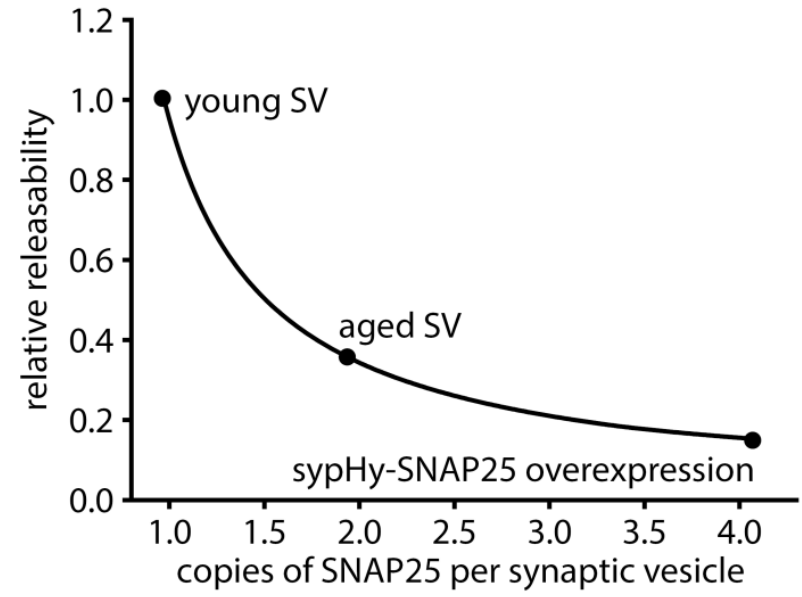

B

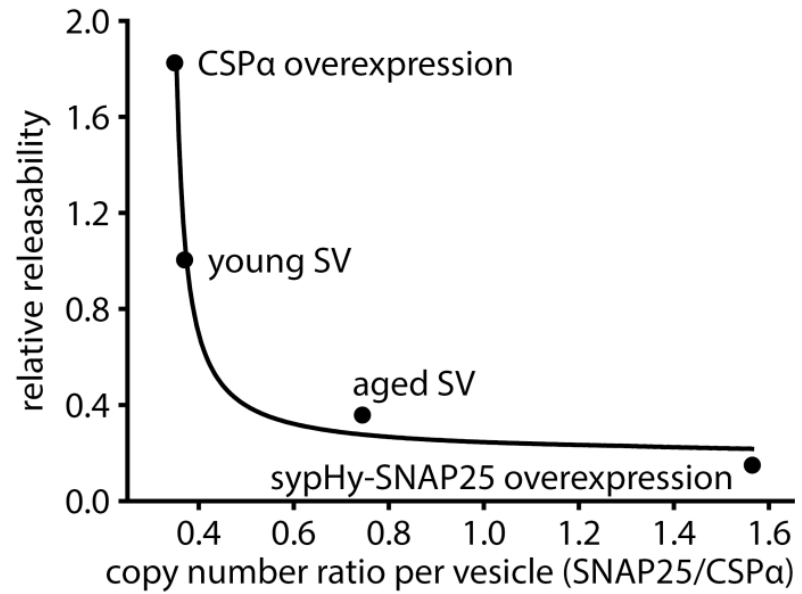

Figure 4.2: The ratio of SNAP25 to CSP on the vesicle predicts releasability in a model compatible with a two-protein interaction.

(A) The relative releasability of a vesicle was calculated by comparison to vesicles that carried the same number of SNAP25 copies as a young synaptic vesicle (SV). For each data point, the experimental condition or the stage in the life cycle of the vesicle for which the numbers were obtained are indicated: young SV, young synaptic vesicles as on day 0 in Figure 3.5C; aged SV, old synaptic vesicle as on day 4 in Figure 3.5C; sypHy-SNAP25 overexpression, vesicle with reduced release as in Figure 3.12. The copy number of SNAP25 per vesicle was predicted as explained in Chapter 4.2, subsection Number of SNAP25 copies on old and on young vesicles: 0.97 and 1.94 respectively. The number of sypHy-SNAP25 was estimated according to Sinha (2011); also see text as above. The fit is a 3-parameter hyperbolic decay, $\mathrm{R}^{2}=1.0$.

(B) The relative releasability of a vesicle was calculated by comparison to vesicles that had the same ratio of SNAP25 copies to CSPa copies as a young synaptic vesicle. For each data point, the experimental condition or the stage in the life cycle of the vesicle for which the numbers were obtained are indicated: CSP overexpression as in vesicles with reduced release in Figure 3.13B; young SV, old SV, sypHy-SNAP25, as in (A). The ratios were derived from the predicted copy numbers of SNAP25 (see (A)) and the average of the published CSPa copy numbers (Takamori et al., 2006; Wilhelm et al., 2014), except for the condition of CSPa overexpression, where the copy number of CSPa per vesicle was estimated from the average copy number of three other overexpressed proteins on synaptic vesicles (Sinha, 2011). The fit is a 3-parameter hyperbolic decay, $R^{2}=0.99$.

Note on the fits: the $\mathrm{R}^{2}$ is of limited value for determining the quality of a fit on only 3-4 data points. The fit is nonetheless useful to describe the general characteristics of the process. The main factor determining the trustworthiness of such a fit is that the data should not be collected over too narrow a range of the independent variable (here: on $\mathrm{x}$-axis). The range here is $\sim 4$ - to 5 -times the minimum. 


\subsection{A timer of organelle usage: SNAP25 contamination and the quantitative CSPa bottleneck}

I have proposed a timer mechanism for synaptic vesicle usage in this Thesis. This timer mechanism inactivates synaptic vesicles after $\sim 260$ rounds of release and recycling. During each round of release, there is a certain chance for the vesicle to pick up SNAP25 from the cell membrane (discussed below), which is $\sim 0.4 \%$ (derived from number of SNAP25 on young and old vesicles and the number of release events per vesicle lifetime). Interestingly, as SNAP25 can only enter synaptic vesicles one full copy at a time, this means that a single contamination event is sufficient to inactivate the vesicle. Once SNAP25 has entered the synaptic vesicle, it causes its inactivation (Figure 3.12). I suggested (Chapter 3.6) that this inactivation is caused by an inhibition of CSPa via cis-interaction with SNAP25 on the vesicle (Figure 3.14). This mechanism will be revisited in this Chapter, and discussed in its relationship to the biophysical possibility of changes in vesicle composition and to the known function of CSPa and SNAP25.

Biophysical constraints of uptake of SNAP25. As discussed in the Introduction (Chapter 1.2), it is unlikely that the synaptic vesicle completely disintegrates upon fusion to the cell membrane, and more probably stays together as a largely coherent entity. This does not, however, exclude the possibility that changes in protein composition could occur over time. Changes in vesicle association with certain cell membrane and endosomal proteins have indeed been observed after recycling of readily releasable vesicles (Hoopmann et al., 2010), although this was not investigated on a longer time scale than a few minutes. In an earlier study, synaptic vesicles were isolated from adult rat brains after perfusion either with highpotassium solution, to increase activity, or with a membrane-permeable calcium chelator, to decrease activity (Burré et al., 2006b). This generated a proteomic snap-shot of recently active and inactive synaptic vesicles, and eight proteins were observed to be changed between these two conditions, with six being increased in quantity and two being decreased by activity. Interestingly, this study also found an increase of SNAP25 on active vesicles, albeit lower than the increase described here. So it seems entirely possible that synaptic vesicles take up or lose certain proteins during recycling. Why then is SNAP25 taken up, specifically, while other and functionally related proteins are not taken up? This question is particularly pressing since there was no increase of syntaxin 1 , which is part of the same SNARE complex as SNAP25, during ageing (Figure 3.11). This might have to do with differences in distributions of SNAP25 and syntaxin 1 in the cell and vesicle membrane. As calculated above (Chapter 4.2), there is, on average, only $\sim 1$ copy of SNAP25 present on young vesicles. By contrast, there are 6.2 copies of syntaxin 1 (which is equally abundant on old and young vesicles, see Figure 3.11) on the average vesicle (Takamori et al., 2006). 
From the total surface area of the synapse and the surface area of the synaptic vesicle $\left(\sim 0.0055 \mu \mathrm{m}^{2}\right)$ and the total copy number of syntaxin 1 and SNAP25 per synapse (Wilhelm et al., 2014), we can derive that the concentration difference between cell membrane and young synaptic vesicle is $\sim 6.4$-fold higher for SNAP25 than for syntaxin 1 . This concentration gradient could act as a sink that draws in SNAP25 to the vesicle membrane to a much higher degree than syntaxin 1. Furthermore, the palmitoylation of SNAP25 might increase its preference for the vesicle patch. SNAP25 does not have a transmembrane domain, but is anchored by up to four closely packed palmitoyl groups (Gonzalo and Linder, 1998; Lane and Liu, 1997). Palmitoyl groups show a marked preference for integration into cholesterolrich membrane patches, such as constituted by synaptic vesicles, especially when the palmitoyl groups are closely packed (Brown and London, 2000). Transmembrane proteins, on the other hand, such as the tail-anchored one-span syntaxin 1 (Bennett et al., 1992), are often excluded from such domains (Brown and London, 2000). It should be noted, however, that this is not a fixed rule that affects all palmitoylated proteins or all transmembrane proteins equally (Brown and London, 2000), and in fact SNAP25 and syntaxin 1 have been observed in the same region of protein clouds on the cell membrane (Saka et al., 2014a). The exclusion effect cannot be too strong in case of syntaxin 1, since the vesicle apparently ultimately takes up more syntaxin 1 during biogenesis than SNAP25 (Takamori et al., 2006). On the other hand, it is also unclear how much opportunity the synaptic vesicle precursor has to take up either SNAP25 or syntaxin 1 during biogenesis. SNAP25 has been observed on intracellular membranes, such as endosomes (Aikawa et al., 2006a; Greaves and Chamberlain, 2011) and the Golgi apparatus (Aikawa et al., 2006a). Syntaxin 1 has also been observed on intracellular membranes (Bajohrs et al., 2005; Kasai and Akagawa, 2001; but also see Aikawa et al., 2006a). To what degree each or any of these proteins co-localize on membranes from which synaptic vesicle precursors originate, has not been investigated yet. It seems clear, however, that the gradient of SNAP25 concentration between cell membrane and young vesicle favours entry of SNAP25 onto the vesicle membrane, while this is not such a strong factor for syntaxin 1.

\section{The role of CSPa in neurotransmission and the quantitative bottleneck. CSPa has} originally been described as a synaptic chaperone with broad activity (Donnelier and Braun, 2014; Evans et al., 2003). More recently, CSPa has mainly been implicated in priming of SNAP25 in a trimeric chaperoning complex with Hsc70 and SGTa (Evans et al., 2003; Sharma et al., 2011, 2012; Thakur et al., 2001). The effects of CSPa knock-outs and CSPa overexpression have been well studied, and support the function of CSP $\alpha$ as a quantitative bottleneck for fusion that I suggested in this Thesis. A knock-out of CSPa initially has relatively mild effects, but mice develop sensor-motor defects after 2-4 weeks, which 
progress until death at around 2 months of age (Fernández-Chacón et al., 2004).

Specifically, muscle tone and muscle mass decrease due to degeneration of neuromuscular junctions, and EPSCs in the Calyx of Held decrease dramatically in number and amplitude, while mEPSCs increase, by P20-P23. Fernández-Chacón et al. (2004) ascribe these effects to a general synaptic chaperoning function of CSPa, since they could not detect any immediate influence on the $\mathrm{Ca}^{2+}$ channels or synaptic vesicle exocytosis itself (EPCSs and mEPSCs are unaltered at P9-P11). According to this hypothesis, synaptic function simply degenerates because of a generalized accumulation of protein damage that would normally be serviced by CSPa. However, taking into account the lifetime of synaptic vesicles in situ, a more specific explanation can be proposed from the data presented in this Thesis. Synaptic phenotypes are first detected at P20-P23, and synaptic vesicles have an estimated lifetime of -20 days in situ (Cohen et al., 2013; Price et al., 2010; Rosenberg et al., 2014; Eugenio F. Fornasiero, personal communication). Note that the published literature allows only a rough estimate here, based mainly on the lifetime of one of the most abundant synaptic vesicle protein, synaptophysin (Price et al., 2010). However, as of yet unpublished results seem to confirm this estimate (Eugenio F. Fornasiero, personal communication). As CSPa knock-outs do not have a timer mechanism that could inactivate ageing synaptic vesicles, they will continue to use them in active release. One would not expect to see severe effects at P9P11, but they should definitely start to occur around P20-P23. The effects observed by Fernández-Chacón et al. (2004) mimic the effects described in this thesis: neurodegeneration (Figure 3.16A,B) and, possibly, a slight reduction and increased unreliability in release (Figure 3.8D). They did not investigate endocytosis phenotypes, as observed here (Figure 3.16C,D). Other studies of CSPa knock-outs observed a reduction of release in response to single action potential stimulation (Rozas et al., 2012; Sharma et al., 2012) as well as endocytosis defects (Rozas et al., 2012). While CSPa knock-outs are associated with reduced levels of SNAP25 (Sharma et al., 2012), and CSPa overexpression is associated with increased levels of SNAP25 (Sharma et al., 2011), which might account for changes in release, this does not explain endocytosis defects. The overexpression of CSPa has also been associated with increased release (Sharma et al., 2011), just as described here (Figure 3.13A,B). The findings in the literature can thus be well reconciled with the model of a timer mechanism under involvement of SNAP25 and CSPa presented here.

Vesicle competition as key constraint of the timer mechanism. The key to interpreting these findings is the realization that the inactivation of ageing synaptic vesicles is not absolute, but always relative to other, younger vesicles. Aged Synaptic vesicles containing increased levels of SNAP25 (Figure 3.11) can still release when younger vesicles are not 
available (Figure 3.8) or have been depleted by high-frequency stimulation (Figure 3.4). They will only be inactivated when out-competed by younger synaptic vesicles with less SNAP25 (Figure 3.5). Young synaptic vesicles are simply more likely to prime successfully than older synaptic vesicles (Figure 4.2), which will lead to young synaptic vesicles preferentially occupying the limited release-sites (Hua et al., 2013; Neher, 2010). This means that ageing synaptic vesicles will become less and less likely to fuse to the cell membrane, spending more and more time in the vesicle cluster in their spherical state with high affinity for synapsin (Krabben et al., 2011), which will increase their propensity to be sequestered in the synapsin/cytoskeleton-meshwork that immobilizes inactive vesicles (compare with Chapter 1.4 in the Introduction). The competition for release-sites between young and old synaptic vesicles is thus the driving force behind the inactivation of the latter. Drastic phenotypes will only occur if this timer mechanism is completely abolished, and after synaptic vesicles have accumulated enough damage to cause trouble when still used in release. If the relative timing of inactivation and degradation of synaptic vesicles derived here for hippocampal cultures (see Chapter 4.2) is transferable to the situation in situ (Cohen et al., 2013; Price et al., 2010; Rosenberg et al., 2014; Eugenio F. Fornasiero, personal communication), inactivation should occur after $\sim 5$ days in situ, with minimal to no damage to the vesicle (Figure 3.17; Chapter 3.7). After 10 days, damage will not exceed $1 \%$ of all vesicle proteins, and minimal to no phenotypes might be detectable yet (P9-P11 in Fernández-Chacón et al., 2004). After 20 days, however, a substantial 6-7\% of vesicle proteins might be damaged and inoperable or performing in unpredictable ways (Chapter 3.7), compatible with the neurodegeneration phenotypes observed in situ (P20-P23 in Fernández-Chacón et al., 2004). This shows that the timer mechanism evolved to incorporate a substantial safety margin of approximately one lifetime in the releasable population, i.e. that synaptic vesicles are inactivated before they actually accumulate significant damage (Figure 3.17) that might compromise the sensitive signaling pathway of neurotransmission. This safety margin is presumably also necessary because only one single contamination event of ageing synaptic vesicles with SNAP25 is enough to inactivate them in competition with younger vesicles (see above). The fact that the contamination is a stochastic event with a probability of $\sim 0.4 \%$ per release event (an average of $\sim 1$ copy of SNAP25 after $\sim 260$ release events) is an additional factor that necessitates a safety margin.

\subsection{Vesicle damage and degradation}

After synaptic vesicle inactivation, they also will have to be degraded at some point; otherwise, there would be no room for newly produced vesicles that replenish the releasable population. I will lay out here how this degradation might proceed via fusion of synaptic vesicles to the endolysosomal system, possibly through direct participation of SNAP25. 
Preceding this part of the discussion, I will lay out the nature of vesicle damage in the synapse and its relation to synaptic activity, and the consequences the accumulation of damage has for neurotransmission.

Accumulation of vesicle damage. The question of accumulation of damage on synaptic vesicles has not yet been studied in any co-ordinated manner. However, accumulation of damage is an inevitability in the life of any protein or organelle. Oxidation from reactive oxygen species produced by mitochondria is a major source of irreversible protein modifications (Davies, 2005; Massaad and Klann, 2013) and is presumably prevalent in the synapse, which is metabolically extremely active (Harris et al., 2012; Laughlin et al., 1998; Rangaraju et al., 2014). Furthermore, the synaptic vesicle is afflicted by frequent and steep variations in $\mathrm{pH}$. Upon fusion, the $\mathrm{pH}$ raises from $\sim 5.5$ to $\sim 7.4$ within milliseconds (Miesenböck et al., 1998; Sankaranarayanan and Ryan, 2000), and it drops back to pH 5.5 with a time constant of $\sim 4-5$ seconds after endocytosis (Atluri and Ryan, 2006). This has the potential to unfold lumenal domains of synaptic vesicle proteins (Talley and Alexov, 2010), which could impair protein function or damage the lumenal assembly of synaptic vesicle proteins (Harlow et al., 2013). This could particularly affect VAMP2, synaptophysin, synaptotagmin 1, and SV2 (Harlow et al., 2013), as well as VGlut and the vATPase, which all have lumenal domains of varying size. A further potential source for protein unfolding arises from the fact that synaptic vesicle proteins can undergo massive conformational changes during their participation in the synaptic vesicle cycle. After a protein has undergone the conformational changes associated with its function, it has to fold back into its original conformation to become functional again. While there are chaperones at the synapse that can presumably assist in refolding (Wyttenbach et al., 2011), this process will likely not be successful in every instance. This manipulation can also be quite drastic and involve the expenditure of significant amounts of energy, such as the unravelling of the SNARE complex through the AAA-ATPase NSF (recently reviewed by Ryu et al., 2016), and might thus be a contributing factor to protein misfolding in itself, although whether SNARE proteins are folded correctly after disassembly of the SNARE complex by NSF remains an open question (Burton and Baker, 2005). It is worth noting here that both disassembly of protein complexes and unfolding of proteins mediated by AAA-ATPases seem to be closely related processes (Vale, 2000) and that NSF can affect other vesicular proteins, such as Rabs, as well (Zhao et al., 2007). The result could be misfolded and non-functional proteins that accumulate on the vesicle. However, this potential source of damage could only affect vesicles while they are still actively working in the releasable population. The following section will therefore investigate vesicle damage in the context of synaptic and metabolic activity. 
Vesicle damage and synaptic activity. As predicted in Figure 3.17, accumulation of damage proceeds mostly after inactivation. It is thus likely that the majority of damage is triggered by oxidation in the metabolically active environment of the synapse. An increase in synaptic activity would necessarily also lead to an increased local energy metabolism, as exocytosis and endocytosis are very energy intensive processes (Harris et al., 2012; Laughlin et al., 1998; Rangaraju et al., 2014), concomitantly increasing the potential for oxidative damage. That synapses are metabolically extremely active environments is evidenced by the fact that mitochondria are present in almost all synapses (Gotow et al., 1991; Palay, 1956; Peters et al., 1976; Waters and Smith, 2003) and account, on average, for $\sim 16 \%$ of the entire synaptic volume (Wilhelm et al., 2014). Local production of ATP is necessary, as diffusion from the cell body would simply take too long to account for local spikes in demand. However, this also means that reactive oxygen species generated locally in response to increased demand due to increased synaptic activity will be relatively confined in the synapse. The potential to damage synaptic vesicles would thus increase with synaptic activity, and the consequently increased metabolic activity. The timer mechanism proposed here (Chapter 4.3) would be ideal to deal with metabolically induced damage, since increased release frequency and increased metabolic activity are directly linked. Vesicle usage is thus a perfect predictor of vesicle damage. Increased usage, which increases the potential for oxidative damage, would also increase the probability within any given timeframe for contamination of synaptic vesicles with SNAP25, due to the more frequent fusion events with the cell membrane. In accordance with this model is the observation that increased usage accelerates inactivation (Figure 3.9). Similar views of damage accumulation served as a framework for the investigation of degenerating synaptic function before (Fernández-Chacón et al., 2004).

\section{Consequences of vesicle damage and removal of damaged proteins and organelles.}

All cellular organelles need to be removed from participation in cellular reactions at some point, of course, or else the accumulated damage on these organelles would disrupt the pathways they are involved in (Sheldrake, 1974; Terman et al., 2007). This is a particularly pressing problem for neurons as post-dividing cells, where accumulated damage cannot be reset by cell division (Nyström and Liu, 2014). Neurons are rarely replaced in mammals after reaching adulthood (Nowakowski, 2006) and thus need to be maintained for as long as possible. This requires sophisticated mechanisms for removal and replacement of damaged organelles. If ageing synaptic vesicles are not removed from release, this can lead to endocytosis defects (Figure 3.16C,D) and ultimately to neurite degeneration (Figure 3.16A,B). A possible explanation for the endocytosis defects comes from the fact that the adaptor machinery of clathrin-mediated endocytosis might attach less efficiently to damaged 
proteins or that re-clustering of synaptic vesicle proteins might become necessary due to decreased cohesion of the vesicle. This would explain the loss of vesicle material into the inter-synaptic axonal membrane (Figure 3.16C,D). While mechanisms have been suggested that might selectively remove misfolded proteins from synaptic vesicles and target them for degradation (Uytterhoeven et al., 2011), there is currently no data available that would support the idea of a local machinery at the synapse that can maintain synaptic vesicle functionality by removing and, critically, replacing non-functional proteins. So the synapse must have evolved different mechanisms of dealing with vesicle damage. The first step, in any classical damage response mechanism, must be the recognition of damage. This can be achieved by synaptic chaperones (Wyttenbach et al., 2011) as well as by the ubiquitin/ESCRT system (Glickman and Ciechanover, 2002; Hochstrasser, 1996; Schmidt and Teis, 2012); the lysosomal/autophagosomal system is usually perceived as more nonselective (Ciechanover, 2005a, 2005b). What signals exactly lead to the recognition of damaged proteins is, remarkably, still largely uncertain (Goldberg, 2003; Hochstrasser, 2006).

A potential role for SNAP25 in vesicle degradation. Synaptic vesicle degradation is generally believed to occur via the endolysosomal system (Candiello et al., 2016; Fernandes et al., 2014b; Haberman et al., 2012; Rizzoli, 2014; Uytterhoeven et al., 2011) and autophagy (Binotti et al., 2014; Rizzoli, 2014; Wang et al., 2015). This was also confirmed in this Thesis, where synaptic vesicles tagged at the synapse appeared in acidic compartments in the cell body after 1-2 days (Figure 3.3). How could ageing synaptic vesicles be specifically fused to the endolysosomal system? Several lines of evidence point to a direct involvement of the $\mathrm{Q}_{\mathrm{bc}}$-SNARE SNAP25, which accumulates on ageing vesicles (Figure 3.11). There are relatively little endosomal $\mathrm{Q}_{\mathrm{bc}}$-SNAREs in the synapse, compared to the number of endosomal $\mathrm{Q}_{\mathrm{a}}$-SNAREs and the vesicular R-SNARE VAMP2 (Wilhelm et al., 2014; see Figure 3.15A). SNAP25 might thus substitute for endosomal $\mathrm{Q}_{\mathrm{bc}}$-SNAREs to mediate fusion of aged vesicles to the endolysosomal system. SNAP25 is known to be quite promiscuous in its interaction with other SNAREs (Bajohrs et al., 2005). It has further been described to facilitate endosomal fusion before (Aikawa et al., 2006b). Additionally, I could observe an increase of Rab7 in synapses overexpressing SNAP25 or sypHy-SNAP25 (Figure 3.15), indicating an increased prevalence of early endolysosomal compartment formation. All these facts point to a direct involvement of SNAP25 in synaptic vesicle degradation. SNAP25 has in fact been described to mediate vesicle fusion to early endosomes in a complex with VAMP2, the synaptic vesicle R-SNARE, and syntaxin 13 (Sun et al., 2003), the most abundant endosomal $Q_{a}$-SNARE at the synapse (Wilhelm et al., 2014; see Figure 3.15A). All this supports the idea of a direct involvement of SNAP25 in synaptic vesicle degradation. 


\subsection{Predicting protein damage and pre-emptive inactivation of organelles: a novel concept for cell biology}

The question remains of why this timer mechanism for synaptic vesicle inactivation evolved. I would like to suggest here that this mechanism is necessary to remove synaptic vesicles from active participation in neurotransmission before they have accumulated significant damage.

\section{"Damage pre-cognition": the need for predicting vesicle damage in}

neurotransmission. However damage and unfolding are recognized, proteins thus affected are usually quite efficiently targeted for degradation (see above). However, for damage to be recognized, it first needs to be present. That means that an organelle that accumulated significant damage might already have participated in cellular reactions. This is less of a problem for the usual cellular pathways, which rely on many hundreds or thousands of functionally identical organelles working continuously and in parallel on the same process. Examples for this type of would be energy metabolism by mitochondria or detoxification by peroxisomes. If a constant small proportion of these organelles are damaged and performing not to the same standards as the undamaged organelles, this will not disrupt the cellular process. In other words, the cell can "afford" to wait for accumulation of damage on these organelles and then recognize the damage and remove the organelles. The other, undamaged, organelles will in the meantime pick up the slack from the damaged organelles and as long as the damaged organelles do not "go rogue" due to the damage, and start to massively disrupt cellular function, the overall process the organelles are involved in will not suffer (or only to a degree that is factored in). However, neurotransmission via synaptic vesicle release is not such a forgiving process. It is not carried by organelles working continuously and in parallel, but by organelles working discontinuously and independently, alone or in small numbers (e.g. $\sim 4.3$ vesicles per activity burst in hippocampal cultures; Figure 3.10I). If an action potential arrives at the synapse, supposed to evoke the release of neurotransmitter, and one of the synaptic vesicles that is activated to perform this function fails to succeed, the entire process of neurotransmission will fail. This can have critical and life-threatening consequences for the organism. It thus has to be absolutely ensured that the synaptic vesicle that is activated during neurotransmission performs its function properly. Could this be done by waiting for damage accumulation that is high enough for damage response mechanisms to recognize and act upon? This is unlikely. Classical damage response mechanisms that target entire organelles do probably not kick in the slightest sign of damage, otherwise an entire organelle might be sacrificed to remove one single damaged protein; this is presumably to inefficient to be compatible with life. So damage response mechanisms evolved to strike a balance between sensitivity and selectivity. And 
this is not a problem for most cellular pathways, relying on organelles working continuously and in parallel, as pointed out above. But for neurotransmission, it might be deadly. Synaptic vesicles that are already damaged enough to lose reliability in release, but not damaged enough yet to be recognized by classical damage response mechanisms, could wreak havoc on the process of neurotransmission. The synapse thus requires a mechanism that removes synaptic vesicles from neurotransmission before they get damaged. How can this be achieved, how can a damage be detected before it actually occurs? The solution lies in a mechanism that times synaptic vesicle usage. This mechanism will have evolved to time synaptic vesicle inactivation to precede the accumulation of significant damage. Precise timing in such a mechanism can have been achieved through parallel evolution with the system of neurotransmission; essentially, the evolution favoured inactivation at a time when no significant damage had occurred yet. The timer mechanism described here is the contamination of SNAP25 on ageing vesicles and the quantitative CSPa bottleneck (Chapter 4.3). The timer can be set to different time points either by adjusting the probability of integration of SNAP25 into the vesicle membrane or by altering the copy number of CSPa on the vesicle; both of these factors are presumably open to evolutionary adjustment. This timer mechanism actually conforms to the main requirement posed here: it removes synaptic vesicles from neurotransmission by inactivation just before they start to accumulate significant damage (Figure 3.17). As one would expect from such a mechanism, it removes vesicles not just directly before they are expected to accumulate damage, but has a margin of error of about one lifetime in the releasable population (see Chapter 3.7).

Conclusion: timing organelle turnover as a new concept for cell biology. The timer mechanism described here, both in its general concept and in its quantitative specifics, constitutes a novel cell biological concept. It is a mechanism that is only useful in cellular mechanisms that require the discontinuous use of a small number of organelles, which is why it might have gone unnoticed until now. These processes are relatively rare in cell biology; most processes in fact rely on continuous parallel activity of many organelles. However, organelles behaving in a similar way to synaptic vesicles affected by the timer mechanism described here have been reported. In bovine adrenal chromaffin cells, the release of large dense-core vesicles (LDCVs) has been reported to correlate inversely with age (Duncan et al., 2003), as has the release of insulin secretory granules in murine pancreatic $\beta$-cells (Ivanova et al., 2013). It is uncertain whether this can be explained by usage, as LDVCs are usually assumed to be one-use organelles, as peptides cannot be transported into them locally after a potential recycling step from the cell membrane; this can only happen during biogenesis of the LDCVs from the Golgi apparatus (Fu et al., 2013). However, a kiss-and-run (for a recent review, see Alabi and Tsien, 2013) type form of 
release has been reported for bovine chromaffin cells (although it has been excluded for pancreatic $\beta$-cells at the same time), which might result in only partial release of the peptide content of the LDVC and thus repeated rounds of release by the same organelle (Ma et al., 2004). It has also been suggested that LDCVs might continue to function in the release of monoamines, which can be transported into LDCVs locally after recycling (Zhang et al., 2006), and that clathrin-mediated bulk-like endocytosis of insulin granules in pancreatic $\beta$ cells is necessary to maintain insulin secretion (Wen et al., 2012). In all three cases, usage could become a factor. Another striking example of removal of aged or dysfunctional organelles has been described for mitochondria, which are sorted according to their functionality during oogenesis in zebrafish (Zhang et al., 2008; Zhou et al., 2010). The mitochondria all try to reach a structure called the mitochondrial cloud, and mitochondria with high inner membrane potential and, consequently, higher ATP-production rate reach there faster, presumably through faster energy-dependent transport along the microtubules leading to the mitochondrial cloud (Zhou et al., 2010). The mechanism of selection is different here, but it is nonetheless a selection mechanism that separates organelles by functionality. It is not, however, a strictly predictive mechanism, since the damage of the non-selected mitochondria is already apparent in their functionality. These are, to my knowledge, the only other examples of organelle sorting based on their functionality. All of them fall short of providing "damage pre-cognition", as the mechanism described here for synaptic vesicles. It is well possible that such a mechanism exists only for synaptic vesicles (but see the discussion of other secretory vesicles above), since they are the only organelle that entirely fits the categorization of working discontinuously and independently from each other, and are at the same time maintaining their identity through several cycles of activity (see Chapter 1.2). For the synaptic vesicle and its behaviour throughout its life cycle, however, which has been outlined in this Thesis, this mechanism presents a powerful way to deal with the problem of damage accumulation on an organelle that the cell cannot afford to use after it has been damaged. 


\section{References}

Abraham, C., Hutter, H., Palfreyman, M.T., Spatkowski, G., Weimer, R.M., Windoffer, R., Jorgensen, E.M., and Leube, R.E. (2006). Synaptic tetraspan vesicle membrane proteins are conserved but not needed for synaptogenesis and neuronal function in Caenorhabditis elegans. Proc. Natl. Acad. Sci. 103, 8227-8232.

Adams, D.J., Arthur, C.P., and Stowell, M.H.B. (2015). Architecture of the Synaptophysin/Synaptobrevin Complex: Structural Evidence for an Entropic Clustering Function at the Synapse. Sci. Rep. 5, 1-9.

Aikawa, Y., Xia, X., and Martin, T. (2006a). SNAP25, but Not Syntaxin 1A, Recycles via an ARF6-regulated Pathway in Neuroendocrine Cells. Mol. Biol. Cell 17, 711-722.

Aikawa, Y., Lynch, K.L., Boswell, K.L., and Martin, T.F.J. (2006b). A Second SNARE Role for Exocytic SNAP25 in Endosome Fusion. Mol. Biol. Cell 17, 2113-2124.

Alabi, A.A., and Tsien, R.W. (2012). Synaptic vesicle pools and dynamics. Cold Spring Harb. Perspect. Biol. 4, 1-18.

Alabi, A.A., and Tsien, R.W. (2013). Perspectives on Kiss-and-Run: Role in Exocytosis, Endocytosis, and Neurotransmission. Annu. Rev. Physiol. 75, 393-422.

Arthur, C.P., and Stowell, M.H.B. (2007). Structure of Synaptophysin: A Hexameric MARVEL-Domain Channel Protein. Structure 15, 707-714.

Atluri, P.P., and Ryan, T.A. (2006). The Kinetics of Synaptic Vesicle Reacidification at Hippocampal Nerve Terminals. J. Neurosci. 26, 2313-2320.

Bajohrs, M., Darios, F., Peak-Chew, S.-Y., and Davletov, B. (2005). Promiscuous interaction of SNAP-25 with all plasma membrane syntaxins in a neuroendocrine cell. Biochem. J. 392, 283-289.

Bal, M., Leitz, J., Reese, A.L., Ramirez, D.M.O., Durakoglugil, M., Herz, J., Monteggia, L.M., and Kavalali, E.T. (2013). Reelin Mobilizes a VAMP7-Dependent Synaptic Vesicle Pool and Selectively Augments Spontaneous Neurotransmission. Neuron 80, 934-946.

Balaji, J., and Ryan, T. a (2007). Single-vesicle imaging reveals that synaptic vesicle exocytosis and endocytosis are coupled by a single stochastic mode. Proc. Natl. Acad. Sci. U. S. A. 104, 20576-20581.

Banker, G.A., and Cowan, W.M. (1977). Rat hippocampal neurons in dispersed cell culture. 
Brain Res. 126, 397-425.

Bauerfeind, R., Régnier-Vigouroux, A.R., Flatmark, T., and Huttner, W.B. (1993). Selective Storage of Acetylcholine, but Not Catecholamines, in Neuroendocrine Microvesicles of Early Endosomal Origin. Neuron 11, 105-121.

Becher, A., Drenckhahn, A., Pahner, I., Margittai, M., Jahn, R., and Ahnert-Hilger, G. (1999). The synaptophysin-synaptobrevin complex: a hallmark of synaptic vesicle maturation. $\mathrm{J}$. Neurosci. 19, 1922-1931.

Belov, L., Matic, K.J., Hallal, S., Best, O.G., Mulligan, S.P., and Christopherson, R.I. (2016). Extensive surface protein profiles of extracellular vesicles from cancer cells may provide diagnostic signatures from blood samples. J. Extracell. Vesicles 1, 1-12.

Benfenati, F., Greengard, P., Brunner, J., and Bahler, M. (1989). Electrostatic and hydrophobic interactions of synapsin I and synapsin I fragments with phospholipid bilayers. J. Cell Biol. 108, 1851-1862.

Bennett, M.K., Calakos, N., Kreiner, T., and Scheller, R.H. (1992). Synaptic vesicle membrane proteins interact to form a multimeric complex. J. Cell Biol. 116, 761-775.

Benson, D., Watkins, F., Steward, O., and Banker, G. (1994). Characterization of GABAergic neurons in hippocampal cell cultures. J. Neurocytol. 23, 279-295.

Binotti, B., Pavlos, N.J., Riedel, D., Wenzel, D., Vorbrüggen, G., Schalk, A.M., Kühnel, K., Boyken, J., Erck, C., Martens, H., et al. (2014). The GTPase RaB26 links synaptic vesicles to the autophagy pathway. Elife 2015, 1-23.

Birks, R., and Maclntosh, F. (1961). Acetylcholine Metabolism of the Sympathetic Ganglion. Can. J. Biochem. Physiol. 39, 787-827.

Blasi, J., Chapman, E.R., Link, E., Binz, T., Yamasaki, S., De Camilli, P., Südhof, T.C., Niemann, H., and Jahn, R. (1993a). Botulinum neutrotoxin A selectively cleaves the synaptic protein SNAP-25. Nature 365, 160-163.

Blasi, J., Chapman, E.R., Yamasaki, S., Binz, T., Niemann, H., and Jahn, R. (1993b). Botulinum neurotoxin $\mathrm{C} 1$ blocks neurotransmitter release by means of cleaving HPC1/syntaxin. EMBO J. 12, 4821-4828.

Blondeau, F., Ritter, B., Allaire, P.D., Wasiak, S., Girard, M., Hussain, N.K., Angers, A., Legendre-guillemin, V., Roy, L., Boismenu, D., et al. (2004). Tandem MS analysis of brain clathrin-coated vesicles reveals their critical involvement in synaptic vesicle recycling. Proc. 
Natl. Acad. Sci. 101, 3833-3838.

Bonanomi, D., Benfenati, F., and Valtorta, F. (2006). Protein sorting in the synaptic vesicle life cycle. Prog. Neurobiol. 80, 177-217.

Brown, D.A., and London, E. (2000). Structure and Function of Sphingolipid- and Cholesterol-rich Membrane Rafts. J. Biol. Chem. 275, 17221-17224.

Brown, D.A., and Rose, J.K. (1992). Sorting of GPI-anchored proteins to glycolipid-enriched membrane subdomains during transport to the apical cell surface. Cell 68, 533-544.

Burré, J., Beckhaus, T., Sägger, H., Corvey, C., Hofmann, S., Karas, M., Zimmermann, H., and Volknandt, W. (2006a). Analysis of the synaptic vesicle proteome using three gel-based protein separation techniques. Proteomics 6, 6250-6262.

Burré, J., Beckhaus, T., Corvey, C., Karas, M., Zimmermann, H., and Volknandt, W. (2006b). Synaptic vesicle proteins under conditions of rest and activation: Analysis by 2-D difference gel electrophoresis. Electrophoresis 3488-3496.

Burton, B.M., and Baker, T.A. (2005). Remodeling protein complexes : Insights from the AAA + unfoldase ClpX and Mu transposase. Protein Sci. 14, 1945-1954.

Cameron, P.L., Südhof, T.C., Jahn, R., and De Camilli, P. (1991). Colocalization of Synaptophysin with Transferrin Receptors: Implications for Synaptic Vesicle Biogenesis. J. Cell 115, 151-164.

Candiello, E., Manuel, K., Wenzel, D., Cassel, D., and Schu, P. (2016). Proteins differentially regulate neuronal early endosome maturation via the Rab5/Vps34-pathway. Sci. Rep. 6, 112.

Cao, Z., Li, C., Higginbotham, J.N., Franklin, J.L., Tabb, D.L., Graves-deal, R., Hill, S., Cheek, K., Jerome, W.G., Lapierre, L.A., et al. (2008). Use of Fluorescence-activated Vesicle Sorting for Isolation of Naked2-associated, Basolaterally Targeted Exocytic Vesicles for Proteomics Analysis. Mol. Cell. Proteomics 7, 1651-1667.

del Castillo, J., and Katz, B. (1954). Quantal components of the end-plate potential. Neuron $124,560-573$.

Ceccarelli, B., Hurlbut, W., and Mauro, A. (1973). Turnover of transmitter and vesicles at the frog neuromuscular junction. J. Cell Biol. 57, 499-524.

Cesca, F., Baldelli, P., Valtorta, F., and Benfenati, F. (2010). Progress in Neurobiology The synapsins : Key actors of synapse function and plasticity. Prog. Neurobiol. 91, 313-348. 
Chamberlain, L.H., Burgoyne, R.D., and Gould, G.W. (2001). SNARE proteins are highly enriched in lipid rafts in PC12 cells: implications for the spatial control of exocytosis. Proc. Natl. Acad. Sci. U. S. A. 98, 5619-5624.

Chen, T.-W., Wardill, T.J., Sun, Y., Pulver, S.R., Renninger, S.L., Baohan, A., Schreiter, E.R., Kerr, R. a, Orger, M.B., Jayaraman, V., et al. (2013). Ultrasensitive fluorescent proteins for imaging neuronal activity. Nature 499, 295-300.

Cheng, X., Zhou, B., Lin, M., Cai, Q., and Sheng, Z. (2015). Axonal autophagosomes recruit dynein for retrograde transport through fusion with late endosomes. J. Cell Biol. 209, 377386.

Chung, C., Barylko, B., Leitz, J., Liu, X., and Kavalali, E.T. (2010). Acute Dynamin Inhibition Dissects Synaptic Vesicle Recycling Pathways That Drive Spontaneous and Evoked Neurotransmission. J. Neurosci. 30, 1363-1376.

Ciechanover, A. (2005a). Intracellular protein degradation: from a vague idea thru the lysosome and the ubiquitin - proteasome system and onto human diseases and drug targeting. Cell Death Differ. 12, 1178-1190.

Ciechanover, A. (2005b). Proteolysis: from the lysosome to ubiquitin and the proteasome. Nat. Rev. Mol. Cell Biol. 6, 79-86.

Cocucci, E., Aguet, F., Boulant, S., and Kirchhausen, T. (2012). The First Five Seconds in the Life of a Clathrin-Coated Pit. Cell 495-507.

Cohen, L.D., Zuchman, R., Sorokina, O., Müller, A., Dieterich, D.C., Armstrong, J.D., Ziv, T., and Ziv, N.E. (2013). Metabolic turnover of synaptic proteins: kinetics, interdependencies and implications for synaptic maintenance. PLoS One 8, e63191.

Craig, A.M., and Banker, G. (1994). Neuronal polarity. Annu. Rev. Neurosci. 17, 267-310.

Cullen, D.K., Gilroy, M.E., Irons, H.R., and Laplaca, M.C. (2010). Synapse-to-neuron ratio is inversely related to neuronal density in mature neuronal cultures. Brain Res. 1359, 44-55.

Daly, C., and Ziff, E.B. (1997). Post-transcriptional regulation of synaptic vesicle protein expression and the developmental control of synaptic vesicle formation. J. Neurosci. 17, 2365-2375.

Darcy, K.J., Staras, K., Collinson, L.M., and Goda, Y. (2006). Constitutive sharing of recycling synaptic vesicles between presynaptic boutons. Nat. Neurosci. 9, 315-321.

Davies, M.J. (2005). The oxidative environment and protein damage. Biochem. Biophys. 
Acta $1703,93-109$.

Deinhardt, K., Salinas, S., Verastegui, C., Watson, R., Worth, D., Hanrahan, S., Bucci, C., Schiavo, G., and Monteroni, P. (2006). Rab5 and Rab7 Control Endocytic Sorting along the Axonal Retrograde Transport Pathway. Neuron 52, 293-305.

Delgado, R., Maureira, C., Oliva, C., Kidokoro, Y., and Labarca, P. (2000). Size of vesicle pools, rates of mobilization, and recycling at neuromuscular synapses of a Drosophila mutant, shibire. Neuron 28, 941-953.

Denker, A., and Rizzoli, S.O. (2010). Synaptic vesicle pools: an update. Front. Synaptic Neurosci. 2, 1-12.

Denker, A., Kröhnert, K., and Rizzoli, S.O. (2009). Revisiting synaptic vesicle pool localization in the Drosophila neuromuscular junction. J. Physiol. 12, 2919-2926.

Denker, A., Bethani, I., Kröhnert, K., Körber, C., Horstmann, H., Wilhelm, B.G., Barysch, S. V, Kuner, T., Neher, E., and Rizzoli, S.O. (2011a). A small pool of vesicles maintains synaptic activity in vivo. Proc. Natl. Acad. Sci. U. S. A. 108, 17177-17182.

Denker, A., Kröhnert, K., Bückers, J., Neher, E., and Rizzoli, S.O. (2011b). The reserve pool of synaptic vesicles acts as a buffer for proteins involved in synaptic vesicle recycling. Proc. Natl. Acad. Sci. U. S. A. 108, 17183-17188.

Deutsch, J.W., and Kelly, R.B. (1981). Lipids of synaptic vesicles: relevance to the mechanism of membrane fusion. Biochemistry 20, 378-385.

Dieterich, D.C., Hodas, J.J.L., Gouzer, G., Shadrin, I.Y., T, J., Triller, A., Tirrell, D.A., and Schuman, E.M. (2011). In situ visualization and dynamics of newly synthesized proteins in rat hippocampal neurons. Nat. Neurosci. 13, 897-905.

Dobson, C.M. (1999). Protein misfolding, evolution and disease. Trends Cell Biol. 24, 329332.

Dobson, C.M. (2003). Protein folding and misfolding. Nature 426, 884-890.

Donnelier, J., and Braun, J.E.A. (2014). CSPa - chaperoning presynaptic proteins. Front. Cell. Neurosci. 8, 1-6.

Duncan, R.R., Greaves, J., Wiegand, U.K., Matskevich, I., Bodammer, G., and Apps, D.K. (2003). Functional and spatial segregation of secretory vesicle pools according to vesicle age. Nature $422,1-5$. 
Elmqvist, D., and Quastel, D.M.J. (1965). A Quantitative Study of End-Plate Potentials in Isolated Human Muscle. J. Physiol. 178, 505-529.

Eshkind, L.G., and Leube, R.E. (1995). Mice lacking synaptophysin reproduce and form typical synaptie vesicles. Cell Tissue Res. 282, 423-433.

Evans, G.J.O., Morgan, A., and Burgoyne, D. (2003). Tying Everything Together: The Multiple Roles of Cysteine String Protein (CSP) in Regulated Exocytosis. Traffic 653-659.

Farías, G.G., Guardia, C.M., Britt, D.J., Guo, X., and Bonifacino, J.S. (2015). Sorting of Dendritic and Axonal Vesicles at the Pre-axonal Exclusion Zone Article Sorting of Dendritic and Axonal Vesicles at the Pre-axonal Exclusion Zone. Cell Rep. 13, 1221-1232.

Fatt, P., and Katz, B. (1950). Some observations on biological noise. Nature 166, 597-598.

Fatt, P., and Katz, B. (1952). Spontaneous subthreshold activity at motor nerve endings. J. Physiol. 117, 109-128.

Feany, M.B., Yee, A.G., Delvy, M.L., and Buckley, K.M. (1993). The Synaptic Vesicle Proteins SV2, Synaptotagmin and Synaptophysin Are Sorted to Separate Cellular Compartments in CHO Fibroblasts. J. Cell Biol. 123, 575-584.

Feng, R., and Konishi, Y. (1992). Analysis of antibodies and other large glycoproteins in the mass range of 150000-200000 Da by electrospray ionization mass spectrometry. Anal. Chem. 64, 2090-2095.

Fernandes, A.C., Uytterhoeven, V., Kuenen, S., Wang, Y.C., Slabbaert, J.R., Swerts, J., Kasprowicz, J., Aerts, S., and Verstreken, P. (2014a). Reduced synaptic vesicle protein degradation at lysosomes curbs TBC1D24/sky-induced neurodegeneration. J. Cell Biol. 207, 453-462.

Fernandes, A.C., Uytterhoeven, V., Kuenen, S., Wang, Y.-C., Slabbaert, J.R., Swerts, J., Kasprowicz, J., Aerts, S., and Verstreken, P. (2014b). Reduced synaptic vesicle protein degradation at lysosomes curbs TBC1D24/sky-induced neurodegeneration. J. Cell Biol. 207, 453-462.

Fernandez-Alfonso, T., and Ryan, T.A. (2008). A heterogeneous "resting" pool of synaptic vesicles that is dynamicall interchanged across boutons in mammalian CNS synapses. Brain Cell Biol. 36, 87-100.

Fernández-Alfonso, T., Kwan, R., and Ryan, T. a (2006). Synaptic vesicles interchange their membrane proteins with a large surface reservoir during recycling. Neuron 51, 179-186. 
Fernández-Chacón, R., Wölfel, M., Nishimune, H., Tabares, L., Schmitz, F., CastellanoMuñoz, M., Rosenmund, C., Montesinos, M.L., Sanes, J.R., Schneggenburger, R., et al. (2004). The synaptic vesicle protein CSPa prevents presynaptic degeneration. Neuron 42, 237-251.

Fornasiero, E.F., and Opazo, F. (2015). Super-resolution imaging for cell biologists. Bioessays 436-451.

Fornasiero, E.F., Bonanomi, D., Benfenati, F., and Valtorta, F. (2010). The role of synapsins in neuronal development. Cell. Mol. Life Sci. 67, 1383-1396.

Fornasiero, E.F., Raimondi, A., Guarnieri, F.C., Orlando, M., Fesce, R., Benfenati, F., and Valtorta, F. (2012). Synapsins Contribute to the Dynamic Spatial Organization of Synaptic Vesicles in an Activity-Dependent Manner. J. Neurosci. 32, 12214-12227.

Fredj, N. Ben, and Burrone, J. (2009). A resting pool of vesicles is responsible for spontaneous vesicle fusion at the synapse. Nat. Neurosci. 12, 751-759.

Fu, Z., Gilbert, E.R., and Liu, D. (2013). Regulation of Insulin Synthesis and Secretion and Pancreatic Beta-Cell Dysfunction in Diabetes. Curr. Diabetes Rev. 9, 25-53.

Gandhi, S.P., and Stevens, C.F. (2003). Three modes of synaptic vesicular recycling revealed by single-vesicle imaging. Nature 423, 607-613.

Geppert, M., Goda, Y., Hammer, R.E., Li, C., Rosahi, T.W., Stevens, C.F., and Siiclhof, T.C. (1994). Synaptotagmin I : A Major Ca2+ Sensor for Transmitter Release at a Central Synapse. Cell 79, 717-727.

Gimber, N., Tadeus, G., Maritzen, T., Schmoranzer, J., and Haucke, V. (2015). Diffusional spread and confinement of newly exocytosed synaptic vesicle proteins. Nat. Commun. 6 , 8392.

Gitler, D., Cheng, Q., Greengard, P., and Augustine, G.J. (2008). Synapsin Ila Controls the Reserve Pool of Glutamatergic Synaptic Vesicles. J. Neurosci. 28, 10835-10843.

Giuditta, A., Kaplan, B.B., Minnen, J. Van, Alvarez, J., Koenig, E., and Giuditta, A. (2002). Axonal and presynaptic protein synthesis: new insights into the biology of the neuron. Trends Neurosci. 25, 400-404.

Glickman, M.H., and Ciechanover, A. (2002). The Ubiquitin-Proteasome Proteolytic Pathway: Destruction for the Sake of Construction. Physiol. Rev. 82, 373-428.

Goldberg, A.L. (2003). Protein degradation and protection against misfolded or damaged 
proteins. Nature 426, 895-899.

Gonzalo, S., and Linder, M.E. (1998). SNAP-25 Palmitoylation and Plasma Membrane Targeting Require a Functional Secretory Pathway. Mol. Biol. Cell 9, 585-597.

Gordon, S.L., Leube, R.E., and Cousin, M.A. (2011). Synaptophysin Is Required for Synaptobrevin Retrieval during Synaptic Vesicle Endocytosis. J. Neurosci. 31, 14032-14036.

Gordon, S.L., Harper, C.B., Smillie, K.J., and Cousin, M.A. (2016). A Fine Balance of Synaptophysin Levels Underlies Efficient Retrieval of Synaptobrevin II to Synaptic Vesicles. PLoS One 11, e0149457.

Gotow, T., Miyaguchi, K., and Hashimoto, P.H. (1991). Cytoplasmic architecture of the axon terminal: filamentous strands specifically associated with synaptic vesicles. Neuroscience 40 , 587-598.

Granseth, B., Odermatt, B., Royle, S., and Lagnado, L. (2006). Clathrin-Mediated Endocytosis Is the Dominant Mechanism of Vesicle Retrieval at Hippocampal Synapses. Neuron 51, 773-786.

Greaves, J., and Chamberlain, L.H. (2011). Differential palmitoylation regulates intracellular patterning of SNAP25. J. Cell Sci. 124, 1351-1360.

Greengard, P., Valtorta, F., Czernik, A.J., and Benfenati, F. (1993). Synaptic Vesicle Phosphoproteins and Regulation of Synaptic Function. Science. 259, 780-785.

Groemer, T.W., and Klingauf, J. (2007). belong to the same vesicle pool. Nat. Neurosci. 10, 145-147.

Haberman, A., Williamson, W.R., Epstein, D., Wang, D., Rina, S., Meinertzhagen, I.A., and Hiesinger, P.R. (2012). The synaptic vesicle SNARE neuronal Synaptobrevin promotes endolysosomal degradation and prevents neurodegeneration. J. Cell Biol. 196, 261-276.

Hannah, M.J., Schmidt, a a, and Huttner, W.B. (1999). Synaptic vesicle biogenesis. Annu. Rev. Cell Dev. Biol. 15, 733-798.

Harata, N., Pyle, J.L., Aravanis, A.M., Mozhayeva, M., Kavalali, E.T., and Tsien, R.W. (2001a). Limited numbers of recycling vesicles in small CNS nerve terminals: Implications for neural signaling and vesicular cycling. Trends Neurosci. 24, 637-643.

Harata, N., Ryan, T.A., Smith, S.J., Buchanan, J., and Tsien, R.W. (2001b). Visualizing recycling synaptic vesicles in hippocampal neurons by FM 1-43 photoconversion. Proc. Natl. Acad. Sci. 98, 12748-12753. 
Harlow, M.L., Szule, J.A., Xu, J., Jung, J.H., Marshall, R.M., and McMahan, U.J. (2013). Alignment of Synaptic Vesicle Macromolecules with the Macromolecules in Active Zone Material that Direct Vesicle Docking. PLoS One 8, e69410.

Harris, J.J., Jolivet, R., and Attwell, D. (2012). Synaptic Energy Use and Supply. Neuron 75, 762-777.

Hering, H., Lin, C., and Sheng, M. (2003). Lipid rafts in the maintenance of synapses, dendritic spines, and surface AMPA receptor stability. J. Neurosci. 23, 3262-3271.

Heuser, J.E., and Reese, T. (1973). Evidence for recycling synaptic vesicle membrane during neurotransmitter release at the frog neuromuscular junction. J. Cell Biol. 57, 315-344.

Hilfiker, S., Pieribone, V.A., Czernik, A.J., Kao, H., Augustine, G.J., and Greengard, P. (1999). Synapsins as regulators of neurotransmitter release. Philos. Trans. R. Soc. London B 354, 269-279.

Hirokawa, N., Niwa, S., and Tanaka, Y. (2010). Molecular Motors in Neurons: Transport Mechanisms and Roles in Brain Function, Development, and Disease. Neuron 68, 610-638.

Hochstrasser, M. (1996). Ubiquitin-dependent protein degradation. Annu. Rev. Genet. 30 , 405-439.

Hochstrasser, M. (2006). Review Lingering Mysteries of Ubiquitin-Chain Assembly. Cell 124, 27-34.

Hoopmann, P., Punge, A., Barysch, S. V, Westphal, V., Bückers, J., Opazo, F., Bethani, I., Lauterbach, M. a, Hell, S.W., and Rizzoli, S.O. (2010). Endosomal sorting of readily releasable synaptic vesicles. Proc. Natl. Acad. Sci. U. S. A. 107, 19055-19060.

Hu, Y., Qu, L., and Schikorski, T. (2008). Mean Synaptic Vesicle Size Varies Among Individual Excitatory Hippocampal Synapses. Synapse 957, 953-957.

Hua, Y., Sinha, R., and Martineau, M. (2010). A common origin of synaptic vesicles undergoing evoked and. Nat. Neurosci. 13, 1451-1453.

Hua, Y., Sinha, R., Thiel, C.S., Schmidt, R., Hüve, J., Martens, H., Hell, S.W., Egner, A., and Klingauf, J. (2011a). A readily retrievable pool of synaptic vesicles. Nat. Neurosci. 14, 833839.

Hua, Y., Woehler, A., Kahms, M., Haucke, V., and Neher, E. (2013). Blocking Endocytosis Enhances Short-Term Synaptic Depression under Conditions of Normal Availability of Vesicles. Neuron 343-349. 
Hua, Z., Leal-Ortiz, S., Foss, S.M., Waites, C.L., Garner, C.C., Voglmaier, S.M., and Edwards, R.H. (2011b). V-SNARE composition distinguishes synaptic vesicle pools. Neuron $71,474-487$.

Ikeda, K., and Bekkers, J.M. (2008). Counting the number of releasable synaptic vesicles in a presynaptic terminal. Proc. Natl. Acad. Sci. U. S. A. 106, 2945-2950.

Ivanova, A., Kalaidzidis, Y., Dirkx, R., Sarov, M., Gerlach, M., Schroth-Diez, B., Müller, A., Liu, Y., Andree, C., Mulligan, B., et al. (2013). Age-dependent labeling and imaging of insulin secretory granules. Diabetes 62, 3687-3696.

Jahn, R., and Fasshauer, D. (2012). Molecular machines governing exocytosis of synaptic vesicles. Nature 490, 201-207.

Jähne, S., Rizzoli, S.O., and Helm, M.S. (2015). The structure and function of presynaptic endosomes. Exp. Cell Res. 335, 172-179.

Janz, R., Su, T.C., Hammer, R.E., Unni, V., Siegelbaum, S.A., and Bolshakov, V.Y. (1999). Essential Roles in Synaptic Plasticity for Synaptogyrin I and Synaptophysin I. Neuron 24, 687-700.

Jia, J., Lamer, S., Schu, M., Schmidt, M.R., Krause, E., and Haucke, V. (2006). Quantitative Proteomics Analysis of Detergent-resistant Membranes from Chemical Synapses. Mol. Cell. Proteomics 4, 2060-2071.

Johnston, P.A., Cameron, P.L., Stukenbrok, H., Jahn, R., De Camilli, P., and Südhof, T.C. (1989). Synaptophysin is targeted to similar microvesicles in $\mathrm{CHO}$ and $\mathrm{PC} 12$ cells. EMBO J. 8, 2863-2872.

Juillerat, A., Gronemeyer, T., Keppler, A., Gendereizig, S., Pick, H., Vogel, H., and Johnsson, K. (2003). Directed Evolution of O6 -Alkylguanine-DNA Alkyltransferase for Efficient Labeling of Fusion Proteins with Small Molecules In Vivo. Chem. Biol. 10, 313-317.

Kaech, S., and Banker, G. (2006). Culturing hippocampal neurons. Nat. Protoc. 1, 24062415.

Kamin, D., Lauterbach, M.A., Westphal, V., Keller, J., Schönle, A., Hell, S.W., and Rizzoli, S.O. (2010). High- and Low-Mobility Stages in the Synaptic Vesicle Cycle. Biophys. J. 99, 675-684.

Kapitein, L.C., and Hoogenraad, C.C. (2011). Which way to go? Cytoskeletal organization and polarized transport in neurons. Mol. Cell. Neurosci. 46, 9-20. 
Karra, D., Dahm, R., Hahn, S., Tu, F., John, P., Vogel, V., Zeitelhofer, M., and Zeitelhofer-, M. (2010). Transfection Techniques for Neuronal Cells. J. Neurosci. 30, 6171-6177.

Kasai, K., and Akagawa, K. (2001). Roles of the cytoplasmic and transmembrane domains of syntaxins in intracellular localization and trafficking. J. Cell Sci. 114, 3115-3124.

Keppler, A., Gendreizig, S., Gronemeyer, T., Pick, H., Vogel, H., and Johnsson, K. (2003). A general method for the covalent labeling of fusion proteins with small molecules in vivo. Nat. Biotechnol. 21, 86-89.

Keppler, A., Pick, H., Arrivoli, C., Vogel, H., and Johnsson, K. (2004). Labeling of fusion proteins with synthetic fluorophores in live cells. Proc. Natl. Acad. Sci. U. S. A. 101, 99559959.

Kim, S.H., and Ryan, T.A. (2010). Article CDK5 Serves as a Major Control Point in Neurotransmitter Release. Neuron 67, 797-809.

Klopfenstein, D.R., and Vale, R.D. (2004). The Lipid Binding Pleckstrin Homology Domain in UNC-104 Kinesin is Necessary for Synaptic Vesicle Transport in Caenorhabditis elegans.

Mol. Biol. Cell 15, 3729-3739.

Klopfenstein, D.R., Tomishige, M., Stuurman, N., Vale, R.D., and Francisco, S. (2002). Role of Phosphatidylinositol(4,5)bisphosphat Organization in Membrane Transport by the Unc104 Kinesin Motor. Cell 109, 347-358.

Kobayashi, T., Storrie, B., Simons, K., and Dotti, C.G. (1992). A functional barrier to movement of lipids in polarized neurons. Nature 359, 647-650.

Körber, C., Horstmann, H., Sätzler, K., and Kuner, T. (2012). Endocytic structures and synaptic vesicle recycling at a central synapse in awake rats. Traffic 13, 1601-1611.

Krabben, L., Fassio, A., Bhatia, V.K., Pechstein, A., Onofri, F., Fadda, M., Messa, M., Rao, Y., Shupliakov, O., Stamou, D., et al. (2011). Synapsin I Senses Membrane Curvature by an Amphipathic Lipid Packing Sensor Motif. J. Neurosci. 31, 18149-18154.

Kraszewski, K., Mundigl, O., Daniell, L., Verderio, C., Matteoli, M., and De Camilli, P. (1995). Synaptic vesicle dynamics in living cultured hippocampal neurons visualized with CY3conjugated antibodies directed against the lumenal domain of synaptotagmin. J. Neurosci. $15,4328-4342$.

Kuromi, H., and Kidokoro, Y. (1998). Two Distinct Pools of Synaptic Vesicles in Single Presynaptic Boutons in a Temperature-Sensitive Drosophila Mutant, shibire. Neuron 20, 
917-925.

Kuromi, H., and Kidokoro, Y. (2000). Tetanic Stimulation Recruits Vesicles from Reserve Pool via a cAMP-Mediated Process in Drosophila Synapses. Neuron 27, 133-143.

Kwon, S.E., and Chapman, E.R. (2011). Synaptophysin regulates the kinetics of synaptic vesicle endocytosis in central neurons. Neuron 70, 847-854.

Lane, S.R., and Liu, Y. (1997). Characterization of the Palmitoylation Domain of SNAP-25. J. Neurochem. 69, 1864-1869.

Lang, T., Bruns, D., Wenzel, D., Riedel, D., Holroyd, P., Thiele, C., and Jahn, R. (2001). SNAREs are concentrated in cholesterol-dependent clusters that define docking and fusion sites for exocytosis. EMBO J. 20, 2202-2213.

de Lange, R.P.J., de Roos, A.D.G., and Borst, J.G.G. (2003). Two Modes of Vesicle Recycling in the Rat Calyx of Held. J. Neurosci. 23, 10164-10173.

Laughlin, S.B., de Ruyter van Steveninck, R.R., and Anderson, J.C. (1998). The metabolic cost of neural information. Nature 1, 36-41.

Lechene, C., Hillion, F., McMahon, G., Benson, D., Kleinfeld, A.M., Kampf, J.P., Distel, D., Luyten, Y., Bonventre, J., Hentschel, D., et al. (2006). High-resolution quantitative imaging of mammalian and bacterial cells using stable isotope mass spectrometry. J. Biol. 5, 1-30.

Leube, R.E., Wiedenmann, B., and Franke, W.W. (1989). Topogenesis and Sorting of Synaptophysin: Synthesis of a Synaptic Vesicle Protein from a Gene Transfected into Nonneuroendocrine Cells. Cell 59, 433-446.

Leube, R.E., Leimer, U., Grund, C., Franke, W.W., Harth, N., and Wiedenmann, B. (1994). Sorting of Synaptophysin into Special Vesicles in Nonneuroendocrine Epithelial Cells. J. Cell Biol. 127, 1589-1601.

Li, Z., and Murthy, V.N. (2001). Visualizing postendocytic traffic of synaptic vesicles at hippocampal synapses. Neuron 31, 593-605.

Lingwood, D., and Simons, K. (2010). Lipid rafts as a membrane-organizing principle. Science $327,46-50$.

Link, E., Edelmann, L., Chou, J.H., Binz, T., Yamasaki, S., Baumer, M., Stidhofs, T.C., Niemann, H., and Jahny, R. (1992). Tetanus Toxin Action : Inhibition of Neurotransmitter Release Linked to Synaptobrevin Proteolysis. Biochem. Biophys. Res. Cummonications 189, 1017-1023. 
Linstedt, A.D., and Kelly, R.B. (1991). Synaptophysin Is Sorted from Endocytotic in Neuroendocrine but Not Transfected Fibroblasts. Neuron 7, 309-317.

Ma, L., Bindokas, V.P., Kuznetsov, A., Rhodes, C., Hays, L., Edwardson, J.M., Ueda, K., Steiner, D.F., and Philipson, L.H. (2004). Direct imaging shows that insulin granule exocytosis occurs by complete vesicle fusion. Proc. Natl. Acad. Sci. U. S. A. 101, 92669271.

Manev, H., Favaron, M., Guidotti, A., and Costa, E. (1989). Delayed Increase of Ca2 Influx Elicited by Glutamate: Role in. Mol. Pharmacol. 36, 106-112.

Marra, V., Burden, J.J., Thorpe, J.R., Smith, I.T., Smith, S.L., Ha, M., Branco, T., and Staras, K. (2012). A Preferentially Segregated Recycling Vesicle Pool of Limited Size Supports Neurotransmission in Native Central Synapses. Neuron 76, 579-589.

Martens, H., Weston, M.C., Boulland, J.-L., Gronborg, M., Grosche, J., Kacza, J., Hoffmann, A., Matteoli, M., Takamori, S., Harkany, T., et al. (2008). Unique Luminal Localization of VGAT-C Terminus Allows for Selective Labeling of Active Cortical GABAergic Synapses. J. Neurosci. 28, 13125-13131.

Massaad, C.A., and Klann, E. (2013). Reactive Oxygen Species in the Regulation of Synaptic Plasticity and Memory. Antioxidants Redox Signal. 14, 2014-2054.

Mathew, S.S., Pozzo-Miller, L., and Hablitz, J.J. (2008). Kainate Modulates Presynaptic GABA Release from Two Vesicle Pools. J. Neurosci. 28, 725-731.

Matouschek, A. (2003). Protein unfolding - an important process in vivo? Curr. Opin. Struct. Biol. 13, 98-109.

Matteoli, M., Takei, K., Perin, M.S., Südhof, T.C., and De Camilli, P. (1992). Exo-endocytotic recycling of synaptic vesicles in developing processes of cultured hippocampal neurons. J. Cell Biol. 117, 849-861.

McIntire, S.L., Reimer, R.J., Schuske, K., Edwards, R., and Jorgensen, E. (1997). Identification and characterization of the vesicular GABA transporter. Nature 389, 870-876. Mcmahon, H.T., Bolshakovt, V.Y., Janz, R., Hammer, R.E., Siegelbaumt, S.A., and li, T.C.S. (1996). Synaptophysin, a major synaptic vesicle protein, is not essential for neurotransmitter release. Proc. Natl. Acad. Sci. U. S. A. 93, 4760-4764.

Michaelson, D.M., Barkai, G., and Barenholz, Y. (1983). Asymmetry of lipid organization in cholinergic synaptic vesicle membranes. Biochem. J. 211, 155-162. 
Miesenböck, G., De Angelis, D. a, and Rothman, J.E. (1998). Visualizing secretion and synaptic transmission with pH-sensitive green fluorescent proteins. Nature 394, 192-195.

Mitter, D., Reisinger, C., Hinz, B., Hollmann, S., Yelamanchili, S. V., Treiber-Held, S., Ohm, T.G., Herrmann, A., and Ahnert-Hilger, G. (2003). The synaptophysin/synaptobrevin interaction critically depends on the cholesterol content. J. Neurochem. 84, 35-42.

Momen-Heravi, F., Balaj, L., Alian, S., Tigges, J., Toxavidis, V., Ericsson, M., Distel, R.J., Ivanov, A.R., Skog, J., and Kuo, W.P. (2012). Alternative methods for characterization of extracellular vesicles. Front. Physiol. 3, 1-8.

Morciano, M., Burre, J., Corvey, C., Karas, M., and Zimmermann, H. (2005). Immunoisolation of two synaptic vesicle pools from synaptosomes : a proteomics analysis. J. Neurochem 95 , 1732-1745.

Mutch, S.A., Kensel-Hammes, P., Gadd, J.C., Fujimoto, B.S., Allen, R.W., Schiro, P.G., Lorenz, R.M., Kuyper, C.L., Kuo, J.S., Bajjalieh, S.M., et al. (2011). Protein Quantification at the Single Vesicle Level Reveals That a Subset of Synaptic Vesicle Proteins Are Trafficked with High Precision. J. Neurosci. 31, 1461-1470.

Nakada, C., Ritchie, K., Oba, Y., Nakamura, M., Hotta, Y., lino, R., Kasai, R.S., Yamaguchi, K., Fujiwara, T., and Kusumi, A. (2003). Accumulation of anchored proteins forms membrane diffusion barriers during neuronal polarization. Nat. Cell Biol. 5, 626-633.

Neher, E. (2010). What is rate-limiting during sustained synaptic activity: vesicle supply or the availability of release sites. Front. Synaptic Neurosci. 2, 1-6.

Neves, G., and Lagnado, L. (1999). The kinetics of exocytosis and endocytosis in the synaptic terminal of goldfish retinal bipolar cells. J. Physiol. 515, 181-202.

Niwa, S., Tanaka, Y., and Hirokawa, N. (2008). KIF1B $\beta$ - and KIF1A-mediated axonal transport of presynaptic regulator Rab3 occurs in a GTP-dependent manner through DENN/MADD. Nat. Cell 10, 1269-1279.

Nowakowski, R.S. (2006). Stable neuron numbers from cradle to grave. Proc. Natl. Acad. Sci. U. S. A. 103, 12219-12220.

Nyström, T., and Liu, B. (2014). The mystery of aging and rejuvenation - a budding topic. Curr. Opin. Microbiol. 18, 61-67.

Okada, Y., Yamazaki, H., Sekine-aizawa, Y., and Hirokawa, N. (1995). The Neuron-Specific Kinesin Superfamily Protein KIF1A Is a Unique Monomeric Motor for Anterograde Axonal 
Transport of Synaptic Vesicle Precursors. Cell 81, 769-780.

Opazo, F., Punge, A., Bückers, J., Hoopmann, P., Kastrup, L., Hell, S.W., and Rizzoli, S.O. (2010). Limited intermixing of synaptic vesicle components upon vesicle recycling. Traffic 11, $800-812$.

Opazo, F., Levy, M., Byrom, M., Schäfer, C., Geisler, C., Groemer, T.W., Ellington, A.D., and Rizzoli, S.O. (2012). Aptamers as potential tools for super-resolution microscopy. Nat. Methods 9, 938-939.

Orenbuch, A., Shalev, L., Marra, V., Sinai, I., Lavy, Y., Kahn, J., Burden, J.J., Staras, K., and Gitler, D. (2012). Synapsin Selectively Controls the Mobility of Resting Pool Vesicles at Hippocampal Terminals. J. Neurosci. 32, 3969-3980.

Otto, H., Hanson, P.I., and Jahn, R. (1997). Assembly and disassembly of a ternary complex of synaptobrevin, syntaxin, and SNAP-25 in the membrane of synaptic vesicles. Proc. Natl. Acad. Sci. U. S. A. 94, 6197-6201.

Owe-Larsson, B., Berglund, M.M., Kristensson, K., Garoff, H., Larhammar, D., Brodin, L., and Löw, P. (1999). Perturbation of the synaptic release machinery in hippocampal neurons by overexpression of SNAP-25 with the Semliki Forest virus vector. Eur. J. Neurosci. 11, 1981-1987.

Paillart, C., Li, J., Matthews, G., and Sterling, P. (2003). Endocytosis and Vesicle Recycling at a Ribbon Synapse. J. Neurosci. 23, 4092-4099.

Palade, G., and Palay, S. (1954). Electron microscope observations of intinterneuronal and neuromuscular synapses. Anat. Rec. 118, 336.

Palay, S.S.L. (1956). Synapses in the central nervous system. J. Biophys. Iochemical Cytol. 2, 193-202.

Pennuto, M., Bonanomi, D., Benfenati, F., and Valtorta, F. (2003). Synaptophysin I Controls the Targeting of VAMP2/Synaptobrevin II to Synaptic Vesicles. Mol. Biol. Cell 14, 49094919.

Peters, A., Palay, S.L., and Webster, H.F. (1976). The Fine Structure of the Nervous System: the Neurons and Supporting Cells (Philadelphia: Saunders).

Pieribone, V.A., Shupliakov, O., Brodin, L., Hilfiker-Rothenfluh, S., Czernik, A.J., and Greengard, P. (1995). Distinct pools of synaptic vesicles in neurotransmitter release. Nature $375,493-497$. 
Piper, M., and Holt, C. (2004). RNA Translation in Axons. Annu. Rev. Cell Dev. Biol. 20, 505-523.

Poirier, M.A., Xiao, W., Macosko, J.C., CHan, C., Shin, Y.-K., and Bennett, M.K. (1998). The synaptic SNARE compley is a parallel four-stranded helical bundle. Nature 5, 765-769.

Prado, V.F., and Prado, M.A.M. (2002). Signals Involved in Targeting Membrane Proteins to Synaptic Vesicles. Cell. Mol. Neurobilogy 22, 565-577.

Prakash, S., and Matouschek, A. (2004). Protein unfolding in the cell. Trends Biochem. Sci. 29, 593-600.

Price, J.C., Guan, S., Burlingame, A., Prusiner, S.B., and Ghaemmaghami, S. (2010). Analysis of proteome dynamis in the mouse brain. Proc. Natl. Acad. Sci. U. S. A. 107, 14508-14513.

Puchkov, D., and Haucke, V. (2013). Greasing the synaptic vesicle cycle by membrane lipids. Trends Cell Biol. 23, 493-503.

Qu, L.E.I., Akbergenova, Y., Hu, Y., and Schikorski, T. (2009). Synapse-to-Synapse Variation in Mean Synaptic Vesicle Size and Its Relationship With Synaptic Morphology and. J. Comp. Neurol. 352, 343-352.

Raingo, J., Khvotchev, M., Liu, P., Darios, F., Li, Y.C., Ramirez, D.M.O., Adachi, M., Lemieux, P., Toth, K., Davletov, B., et al. (2012). VAMP4 directs synaptic vesicles to a pool that selectively maintains asynchronous neurotransmission. Nat. Neurosci. 15, 738-746.

Ramirez, D.M.O., Khvotchev, M., Trauterman, B., and Kavalali, E.T. (2012). Vti1a Identifies a Vesicle Pool that Preferentially Recycles at Rest and Maintains Spontaneous Neurotransmission. Neuron 73, 121-134.

Rangaraju, V., Calloway, N., and Ryan, T.A. (2014). Activity-Driven Local ATP Synthesis Is Required for Synaptic Function. Cell 156, 825-835.

Regnier-Vigouroux, A., Tooze, S.A., and Huttnerl, W.B. (1991). Newly synthesized synaptophysin is transported to synaptic-like microvesicles via constitutive secretory vesicles and the plasma membrane. EMBO J. 10, 3589-3601.

Revelo, N.H., Kamin, D., Truckenbrodt, S., Wong, A.B., Reuter-Jessen, K., Reisinger, E., Moser, T., and Rizzoli, S.O. (2014). A new probe for super-resolution imaging of membranes elucidates trafficking pathways. J. Cell Biol. 205, 591-606.

Richards, D.A., Guatimosim, C., and Betz, W.J. (2000). Two Endocytic Recycling Routes 
Selectively Fill Two Vesicle Pools in Frog Motor Nerve Terminals. Neuron 27, 551-559.

Richards, D.A., Guatimosim, C., Rizzoli, S.O., and Betz, W.J. (2003). Synaptic vesicle pools at the frog neuromuscular junction. Neuron 39, 529-541.

Rizzoli, S.O. (2014). Synaptic vesicle recycling: steps and principles. EMBO J. 33, 788-822.

Rizzoli, S.O., and Betz, W.J. (2004). The structural organization of the readily releasable pool of synaptic vesicles. Science. 303, 2037-2039.

Rizzoli, S.O., and Betz, W.J. (2005). Synaptic vesicle pools. Nat. Rev. Neurosci. 6, 57-69.

Rizzoli, S.O., Bethani, I., Zwilling, D., Wenzel, D., Siddiqui, T.J., Brandhorst, D., and Jahn, R. (2006). Evidence for early endosome-like fusion of recently endocytosed synaptic vesicles. Traffic 7, 1163-1176.

De Robertis, E.D., and Bennett, H.S. (1955). Some features of the submicroscopic morphology of synapses in frog and earthworm. J. Biophys. Biochem. Cytol. 1, 47-58.

Rohrbough, J., and Broadie, K. (2005). Lipid regulation of the synaptic vesicle cycle. Nat. Rev. Neurosci. 6, 139-150.

Roos, J., and Kelly, R.B. (1999). The endocytic machinery in nerve terminals surrounds sites of exocytosis. Curr. Biol. 9, 1411-1414.

Rose, T., Schoenenberger, P., Jezek, K., and Oertner, T.G. (2013). Developmental Refinement of Vesicle Cycling at Schaffer Collateral Synapses. Neuron 77, 1109-1121.

Rosenberg, T., Gal-Ben-Ari, S., Dieterich, D.C., Kreutz, M.R., and Ziv, N.E. (2014). The roles of protein expression in synaptic plasticity and memory consolidation. Front. Mol. Neurosci. 7, 1-14.

Rostovtsev, V. V., Green, L.G., Fokin, V. V., and Sharpless, K.B. (2002). A stepwise huisgen cycloaddition process: Copper(I)-catalyzed regioselective "ligation" of azides and terminal alkynes. Angew. Chemie - Int. Ed. 41, 2596-2599.

Rozas, J.L., Gómez-Sánchez, L., Mircheski, J., Linares-Clemente, P., Nieto-González, J.L., Vázquez, M.E., Luján, R., and Fernández-Chacón, R. (2012). Motorneurons require cysteine string protein- $\alpha$ to maintain the readily releasable vesicular pool and synaptic vesicle recycling. Neuron 74, 151-165.

Rubinsztein, D.C. (2006). The roles of intracellular protein-degradation pathways in neurodegeneration. Nature 443, 780-786. 
Ryu, J., Jahn, R., and Yoon, T. (2016). Review Progresses in Understanding NEthylmaleimide Sensitive Factor (NSF) Mediated Disassembly of SNARE Complexes. Biopolymers 105, 518-531.

Saka, S.K., Honigmann, A., Eggeling, C., Hell, S.W., Lang, T., and Rizzoli, S.O. (2014a). Multi-protein assemblies underlie the mesoscale organization of the plasma membrane. Nat. Commun. 5, 1-14.

Saka, S.K., Vogts, A., Kröhnert, K., Hillion, F., Rizzoli, S.O., and Wessels, J.T. (2014b). Correlated optical and isotopic nanoscopy. Nat. Commun. 5, 3664.

Sankaranarayanan, S., and Ryan, T. a (2000). Real-time measurements of vesicle-SNARE recycling in synapses of the central nervous system. Nat. Cell Biol. 2, 197-204.

Sara, Y., Virmani, T., Deák, F., Liu, X., and Kavalali, E.T. (2005). An Isolated Pool of Vesicles Recycles at Rest and Drives Spontaneous Neurotransmission. Neuron 45, 563 573.

Schiavo, G., Benfenati, F., Poulain, B., de Laureto, P.P., DasGuptal, B.R., and Montecucco, C. (1992). Tetanus and botulinum-B neurotoxins block neurotransmitter release by proteolytic cleavage of synaptobrevin. Nature 359, 832-835.

Schikorski, T., and Stevens, C.F. (1997). Quantitative ultrastructural analysis of hippocampal excitatory synapses. J. Neurosci. 17, 5858-5867.

Schikorski, T., and Stevens, C.F. (2001). Morphological correlates of functionally defined synaptic vesicle populations. Nat. Neurosci. 4, 391-395.

Schmidt, O., and Teis, D. (2012). The ESCRT machinery. Curr. Biol. 22, 116-120.

Sharma, M., Burré, J., and Südhof, T.C. (2011). CSPa promotes SNARE-complex assembly by chaperoning SNAP-25 during synaptic activity. Nat. Cell Biol. 13, 30-39.

Sharma, M., Burré, J., Bronk, P., Zhang, Y., Xu, W., and Südhof, T.C. (2012). CSPa knockout causes neurodegeneration by impairing SNAP-25 function. EMBO J. 31, 829-841.

Sheehan, P., Zhu, M., Beskow, A., Vollmer, X.C., and Waites, X.C.L. (2016). ActivityDependent Degradation of Synaptic Vesicle Proteins Requires Rab35 and the ESCRT Pathway. J. Neurophysiol. 36, 8668-8686.

Sheldrake, A. (1974). The ageing, growth and death of cells. Nature 250, 381-385.

Simons, K., and Ikonen, E. (1997). Functional rafts in cell membranes. Nature 387, 569-572. 
Sinha, R. (2011). Optical analysis of synaptic vesicle protein molecules during exo- and endocytosis using pH-switchable fluorescent probes.

Sinha, R., Ahmed, S., Jahn, R., and Klingauf, J. (2011). Two synaptobrevin molecules are sufficient for vesicle fusion in central nervous system synapses. Proc. Natl. Acad. Sci. U. S. A. $108,14318-14323$.

Söllner, T., Bennett, M.K., Whiteheart, S.W., Scheller, R.H., and Rothman, J.E. (1993). A Protein Assembly-Disassembly Pathway In Vitro That May Correspond to Sequential Steps of Synaptic Vesicle Docking, Activation, and Fusion. Cell 75, 409-418.

Song, S., and Augustine, G.J. (2015). Synapsin Isoforms and Synaptic Vesicle Trafficking. Mol. Cells 38, 936-940.

Song, A., Wang, D., Chen, G., Li, Y., Luo, J., Duan, S., and Poo, M. (2009). A Selective Filter for Cytoplasmic Transport at the Axon Initial Segment. Cell 136, 1148-1160.

Staras, K., Branco, T., Burden, J.J., Pozo, K., Darcy, K., Marra, V., and Ratnayaka, A. (2010). Report Multiple Presynaptic Terminals in Hippocampal Neurons. Neuron 66, 37-44.

Steinhauser, M.L., and Lechene, C.P. (2013). Quantitative imaging of subcellular metabolism with stable isotopes and multi-isotope imaging mass spectrometry. Semin. Cell Dev. Biol. 24, 661-667.

Stevens, C.F., and Williams, J.H. (2007). Discharge of the readily releasable pool with action potentials at hippocampal synapses. J. Neurophysiol. 98, 3221-3229.

Stevens, R.J., Akbergenova, Y., Jorquera, R.A., and Littleton, J.T. (2013). Abnormal Synaptic Vesicle Biogenesis in Drosophila Synaptogyrin Mutants. J. Neurosci. 32, 1805418067.

Steward, O., and Schuman, E.M. (2003). Compartmentalized Synthesis and Degradation of Proteins in Neurons. Neuron 40, 347-359.

Südhof, T.C. (1995). The synaptic vesicle cycle: a cascade of protein-protein interactions. Nature 375, 645-653.

Südhof, T.C. (2004). The synaptic vesicle cycle. Annu. Rev. Neurosci. 27, 509-547.

Südhof, T.C., and Rizo, J. (2011). Synaptic Vesicle Exocytosis. Cold Spring Harb. Perspect. Biol. 1-14.

Sun, W., Yan, Q., Vida, T.A., and Bean, A.J. (2003). Hrs regulates early endosome fusion by 
inhibiting formation of an endosomal SNARE complex. J. Cell Biol. 162, 125-137.

Sutton, R.B., Fasshauer, D., Jahn, R., and Brunger, A.T. (1998). Crystal structure of a SNARE complex involved in synaptic expcytosis at $2.4 \AA$ resolution. Nature 395, 347-353.

Takamori, S., Holt, M., Stenius, K., Lemke, E. a, Grønborg, M., Riedel, D., Urlaub, H., Schenck, S., Brügger, B., Ringler, P., et al. (2006). Molecular anatomy of a trafficking organelle. Cell 127, 831-846.

Talley, K., and Alexov, E. (2010). On the pH-optimum of activity and stability of proteins. Proteins 78, 2699-2706.

Teng, H., and Wilkinson, R.S. (2000). Clathrin-Mediated Endocytosis near Active Zones in Snake Motor Boutons. J. Neurosci. 20, 7986-7993.

Terman, A., Gustafsson, B., and Brunk, U. (2007). Autophagy, organelles and ageing. J. Pathol. 211, 134-143.

Thakur, P., Su, T.C., Rettig, J., and Stahl, B. (2001). A Trimeric Protein Complex Functions as a Synaptic Chaperone Machine. Neuron 31, 987-999.

Thiele, C., Hannah, M.J., Fahrenholz, F., and Huttner, W.B. (2000). Cholesterol binds to synaptophysin and is required for biogenesis of synaptic vesicles. Nat. Cell Biol. 2, 42-49.

Truckenbrodt, S., and Rizzoli, S.O. (2014). Spontaneous vesicle recycling in the synaptic bouton. Front. Cell. Neurosci. 8.

Truckenbrodt, S., and Rizzoli, S.O. (2015). Synaptic vesicle pools: Classical and emerging roles.

Uytterhoeven, V., Kuenen, S., Kasprowicz, J., Miskiewicz, K., and Verstreken, P. (2011). Loss of Skywalker reveals synaptic endosomes as sorting stations for synaptic vesicle proteins. Cell 145, 117-132.

Vale, R.D. (2000). AAA Proteins: Lords of the Ring. J. Cell Biol. 150, 13-19.

Vale, R.D. (2003). The Molecular Motor Toolbox for Intracellular Transport. Cell 112, 467480.

Valtorta, F., Pozzi, D., Benfenati, F., and Fornasiero, E.F. (2011). The synapsins: Multitask modulators of neuronal development. Semin. Cell Dev. Biol. 22, 378-386.

Verstegen, A.M.J., Tagliatti, E., Lignani, G., Marte, A., Stolero, T., Atias, M., Corradi, A., Valtorta, F., Gitler, D., Onofri, F., et al. (2014). Phosphorylation of Synapsin I by Cyclin- 
Dependent Kinase- 5 Sets the Ratio between the Resting and Recycling Pools of Synaptic Vesicles at Hippocampal Synapses. J. Neurosci. 34, 7266-7280.

Verstreken, P., Ohyama, T., Haueter, C., Habets, R.L.P., Lin, Y.Q., Swan, L.E., Ly, C. V, Venken, K.J.T., Camilli, P. De, and Bellen, H.J. (2009). Tweek, an Evolutionarily Conserved Protein, Is Required for Synaptic Vesicle Recycling. Neuron 63, 203-215.

Wagner, O.I., Esposito, A., Köhler, B., Chen, C.-W., Shen, C.-P., Wu, G.-H., Butkevich, E., Mandalapu, S., Wenzel, D., Wouters, F.S., et al. (2009). Synaptic scaffolding protein SYD-2 clusters and activates kinesin-3 UNC-104 in C. elegans. Proc. Natl. Acad. Sci. U. S. A. 106, 19605-19610.

Wang, T., Martin, X.S., Papadopulos, A., Harper, C.B., Mavlyutov, T.A., Niranjan, D., Glass, N.R., Cooper-white, J.J., Sibarita, J., Choquet, D., et al. (2015). Control of Autophagosome Axonal Retrograde Flux by Presynaptic Activity Unveiled Using Botulinum Neurotoxin Type A. J. Neurosci. 35, 6179-6194.

Watanabe, S., Liu, Q., Davis, M.W., Hollopeter, G., Thomas, N., Jorgensen, N.B., and Jorgensen, E.M. (2013a). Ultrafast endocytosis at Caenorhabditis elegans neuromuscular junctions. Elife 2, e00723.

Watanabe, S., Rost, B.R., Camacho-Pérez, M., Davis, M.W., Söhl-Kielczynski, B., Rosenmund, C., and Jorgensen, E.M. (2013b). Ultrafast endocytosis at mouse hippocampal synapses. Nature 504, 242-247.

Watanabe, S., Trimbuch, T., Camacho-Pérez, M., Rost, B.R., Brokowski, B., SöhlKielczynski, B., Felies, A., Davies, M.W., Rosenmund, C., and Jorgensen, E.M. (2014). Clathrin regenerates synaptic vesicles from endosomes. Nature 228-233.

Waters, J., and Smith, S.J. (2003). Mitochondria and release at hippocampal synapses. Eur. J. Physiol. 447, 363-370.

Welzel, O., Knörr, J., Stroebel, A.M., Kornhuber, J., and Groemer, T. (2011). A fast and robust method for automated analysis of axonal transport. Eur. Biophys. J. 40, 1061-1069.

Wen, D., Xue, Y., Liang, K., Yuan, T., Lu, J., Zhao, W., Xu, T., and Chen, L. (2012). Bulk-like endocytosis plays an important role in the recycling of insulin granules in pancreatic beta cells. Protein Cell 3, 618-626.

Westphal, V., Rizzoli, S.O., Lauterbach, M.A., Kamin, D., Jahn, R., and Hell, S.W. (2008). Video-Rate Far-Field Optical Nanoscopy Dissects Synaptic Vesicle Movement. Science. 320, 246-249. 
Wienisch, M., and Klingauf, J. (2006). Vesicular proteins exocytosed and subsequently retrieved by compensatory endocytosis are nonidentical. Nat. Neurosci. 9, 1019-1027. Wilhelm, B.G., Groemer, T.W., and Rizzoli, S.O. (2010). The same synaptic vesicles drive active and spontaneous release. Nat. Neurosci. 13, 1454-1456.

Wilhelm, B.G., Mandad, S., Truckenbrodt, S., Kröhnert, K., Schäfer, C., Rammner, B., Koo, S.J., Claßen, G.A., Krauss, M., Haucke, V., et al. (2014). Composition of isolated synaptic boutons reveals the amounts of vesicle trafficking proteins. Science. 344, 1023-1028.

Willig, K.I., Rizzoli, S.O., Westphal, V., Jahn, R., and Hell, S.W. (2006). STED microscopy reveals that synaptotagmin remains clustered after synaptic vesicle exocytosis. Nature 440 , 935-939.

Winckler, B., Forscher, P., and Mellman, I. (1999). letters to nature A diffusion barrier maintains distribution of membrane proteins in polarized neurons. Nature 397, 698-701.

Wragg, R.T., Snead, D., Dong, Y., Ramlall, T.F., Menon, I., Bai, J., Eliezer, D., and Dittman, J.S. (2013). Article Synaptic Vesicles Position Complexin to Block Spontaneous Fusion. Neuron 77, 323-334.

Wu, Y.E., Huo, L., Maeder, C.I., Feng, W., and Shen, K. (2013). The Balance Between Capture and Dissociation of Presynaptic Proteins Controls the Spatial Distribution of Synapses. Neuron 78, 994-1011.

Wyttenbach, A., Quraishe, S., Bailey, J., and Connor, V.O. (2011). Molecular Chaperones in the Mammalian Brain: Regional Distribution, Cellular Compartmentalization and Synaptic Interactions. In Folding for the Synapse, pp. 123-144.

Xu-Friedman, M.A., Harris, K.M., and Regehr, W.G. (2001). Three-dimensional comparison of ultrastructural characteristics at depressing and facilitating synapses onto cerebellar Purkinje cells. J. Neurosci. 21, 6666-6672.

Zanetti, M.N., Bello, O.D., Wang, J., Coleman, J., Cai, Y., Sindelar, C. V, Rothman, J.E., and Krishnakumar, S.S. (2016). Ring-like oligomers of Synaptotagmins and related C2 domain proteins. Elife 5, e17262.

Zenisek, D., Steyer, J.A., Feldman, M.E., and Almers, W. (2002). A membrane marker leaves synaptic vesicles in milliseconds after exocytosis in retinal bipolar cells. Neuron 35, 10851097.

Zhang, B., Koh, Y.H., Beckstead, R.B., Budnik, V., Ganetzky, B., Bellen, H.J., and Hughes, 
H. (1998). Synaptic Vesicle Size and Number Are Regulated by a Clathrin Adaptor Protein Required for Endocytosis. Neuron 21, 1465-1475.

Zhang, Y.Z., Ouyang, Y.C., Hou, Y., Schatten, H., Chen, D.Y., and Sun, Q.Y. (2008).

Mitochondrial behavior during oogenesis in zebrafish: A confocal microscopy analysis. Dev. Growth Differ. 50, 189-201.

Zhang, Z., Wu, Y., Wang, Z., Dunning, F.M., Rehfuss, J., and Brennwald, P. (2006). Release mode of large and small dense-core vesicles specified by different synaptotagmin isoforms in PC12 cells. Mol. Biol. Cell 22, 2324-2336.

Zhao, C., Takita, J., Tanaka, Y., Setou, M., Nakagawa, T., Takeda, S., Yang, H.W., Terada, S., Nakata, T., Takei, Y., et al. (2001). Charcot-Marie-Tooth Disease Type 2A Caused by Mutation in a Microtubule Motor KIF1B. Cell 105, 587-597.

Zhao, C., Slevin, J.T., and Whiteheart, S.W. (2007). Cellular Functions of NSF: Not Just SNAPs and SNAREs. FEBS Lett. 581, 2140-2149.

Zhou, R.R., Wang, B., Wang, J., Schatten, H., and Zhang, Y.Z. (2010). Is the mitochondrial cloud the selection machinery for preferentially transmitting wild-type mtDNA between generations? Rewinding Müller's ratchet efficiently. Curr. Genet. 56, 101-107. 


\section{List of Figures}

Figure 1.1: Synaptic vesicles maintain their cohesion upon fusion and only lose a minor fraction of their protein components.

Figure 1.2: The three classical functionally distinct synaptic vesicle pools.

Figure 1.3: The three major non-classical synaptic vesicle pools.

Figure 1.4: The distinctions and commonalities of synaptic vesicle pools.

Figure 1.5: The synaptic vesicle life cycle.

Figure 3.1: Synaptic vesicles can be reliably tagged with antibodies and followed for up to 10 day in living hippocampal cultures.

Figure 3.2: Live-tagging of synaptotagmin 1 in culture labels the complete releasable population of synaptic vesicles.

Figure 3.3: Live-tagged synaptic vesicles recapitulate the normal vesicle life cycle, including degradation in lysosomes.

Figure 3.4: Synaptic vesicles lose the ability to release as they age.

Figure 3.5: Synaptic vesicles become inactive within less than a day during intrinsic network activity.

Figure 3.6: A novel VAMP2-based genetic reporter of synaptic vesicle ageing confirms that young synaptic vesicles are preferentially released.

Figure 3.7: Releasable synaptic vesicles are metabolically young.

Figure 3.8: The synapse is critically dependent on newly produced synaptic vesicles to replenish the releasable population.

Figure 3.9: Synaptic vesicle inactivation is not determined by passing time, but by usage. 
Figure 3.10: Live-cell imaging of synaptic activity and synaptic vesicle release allows the calculation of the number of release events per synaptic vesicle lifetime.

Figure 3.11: Ageing synaptic vesicles get contaminated with SNAP25 from the cell membrane.

Figure 3.12: SNAP25 on synaptic vesicles results in their inactivation.

Figure 3.13: CSPa is a quantitative bottleneck for synaptic vesicle release and its interaction with vesicular SNAP25 acts as a timer for vesicle inactivation after $\sim 260$ uses.

Figure 3.14: A model of the timer mechanism.

Figure 3.15: SNAP25 contamination of synaptic vesicles coincides with the fusion of synaptic vesicles to early endo-/lysosomal compartments.

Figure 3.16: Using aged synaptic vesicles leads to neurodegeneration and endocytosis defects.

Figure 3.17: Synaptic vesicle inactivation via the timer mechanism pre-empts damage.

Figure 4.1: The synaptic vesicle life cycle revisited.

Figure 4.2: The ratio of SNAP25 to CSP on the vesicle predicts releasability in a model compatible with a two-protein interaction. 


\section{List of Tables}

Table 1.1: Surface resident fractions of major synaptic vesicle proteins and synaptic vesicleassociated proteins.

Table 2.1: Enzyme solution for isolation of neuronal precursor cells from rat hippocampi (solution equilibrated with carbogen for $10 \mathrm{~min}$ and sterile filtered before use).

Table 2.2: Enzyme inactivating solution for isolation of neuronal precursor cells from rat hippocampi.

Table 2.3: Plating medium for seeding isolated hippocampal neuronal precursor cells on coverslips.

Table 2.4: Rat hippocampal neuron culture medium.

Table 2.5: Tyrode's solution for washing steps of living neuronal cultures ( $\mathrm{pH}$ adjusted to 7.4 and sterile filtered before use).

Table 2.6: Phosphate buffered saline (PBS), pH adjusted to 7.3.

Table 2.7: pH 5.5 buffer for CypHer5E imaging.

Table 2.8: $\mathrm{pH} 7.4$ buffer for CypHer5E imaging.

Table 2.9: Staining solution for post-fixation immunostainings.

Table 2.10: Blocking solution for post-fixation immunostainings.

Table 2.11: High-salt PBS.

Table 2.12: Mowiol solution (prepared by heating to $50-60^{\circ} \mathrm{C}$ ).

Table 2.13: List of primary antibodies used for post-fixation immunostainings (all diluted from a $1 \mathrm{mg} / \mathrm{ml}$ stock). The antibodies were from Synaptic Systems, Göttingen, Germany or Cell Signalling Technology, Cambridge, UK. 
Table 2.14: List of secondary antibodies used for post-fixation immunostainings (all diluted from an $\sim 0.4 \mathrm{mg} / \mathrm{ml}$ stock). The antibodies were from Dianova, Hamburg, Germany or Active Motif, Carlsbad, CA, USA.

Table 2.15: Methionine-free medium for labelling of newly produced proteins with AHA.

Table 2.16: Melamine solution (mixed by agitation at $250 \mathrm{rpm}$ on a horizontal shaker until melamine was completely dissolved).

Table 2.17: Reaction mix for PCR amplification of DNA.

Table 2.18: PCR programme for DNA amplification, steps 2)-4) repeated 30x.

Table 2.19: Reaction mix for DNA restriction digest.

Table 2.20: Reaction mix for DNA ligation.

Table 2.21: Fresh DMEM (fDMEM) for transfection of neurons (adjusted to $\mathrm{pH} 7.5$ before use).

Table 2.22: Transfection mix for one 12-well plate of rat hippocampal neurons (HBS added last, followed by thorough vortexing for $\sim 30 \mathrm{~s}$ ). 


\section{List of Equations}

(1) Calculation of the number of release events per synaptic vesicle lifetime in hippocampal cultures.

(2) Calculation of the rate of synaptic vesicle production in cultured hippocampal neurons.

(3) Calculation of the copy number of SNAP25 on young, releasable synaptic vesicles.

(4) Calculation of the copy number of SNAP25 on old, inactive synaptic vesicles. 


\section{List of Abbreviations}

\begin{tabular}{|c|c|}
\hline ADP & adenosine diphosphate \\
\hline $\mathrm{AHA}$ & L-azidohomoalanine \\
\hline ANOVA & analysis of variance \\
\hline Arf & ADP ribosylation factor \\
\hline ARL-8 & Arf-like small G protein 8 \\
\hline ATP & adenosine triphosphate \\
\hline$A Z$ & active zone \\
\hline BSA & bovine serum albumin \\
\hline COIN & correlated optical and isotopic nanoscopy \\
\hline CSP & cysteine string protein \\
\hline Cys & L-cysteine \\
\hline DENN & differentially expressed in normal and neoplastic cells \\
\hline DMEM & Dulbecco's modified eagle medium \\
\hline DNA & deoxyribonucleic acid \\
\hline ER & endoplasmic reticulum \\
\hline EPSC & evoked post-synaptic current \\
\hline ESCRT & endosomal sorting complexes required for transport \\
\hline FACS & fluorescence activated cell sorting \\
\hline FAVS & fluorescence activated vesicle sorting \\
\hline FCS & fetal calf serum \\
\hline FUNCAT & fluorescent non-canonical amino acid tagging \\
\hline fDMEM & fresh Dulbecco's Modified Eagle Medium \\
\hline Gln & L-glutamine \\
\hline HBS & HEPES buffered saline \\
\hline HEPES & 4-(2-hydroxyethyl)-1-piperazineethanesulfonic acid \\
\hline JNK & c-Jun N-terminal kinase \\
\hline LDCV & large dense-core vesicle \\
\hline MADD & MAP kinase-activating death domain \\
\hline MAP kinase & mitogen-activated protein kinase \\
\hline MAVS & magnetism activated vesicle sorting \\
\hline MEM & minimum essential medium \\
\hline mEPSC & miniature evoked post-synaptic current \\
\hline Met & L-methionine \\
\hline NA & numerical aperture \\
\hline nanoSIMS & nanoscale secondary ion mass spectrometry \\
\hline
\end{tabular}


$\mathrm{NMJ}$ neuromuscular junction

NSF N-ethylmaleimide sensitive factor

$\mathrm{P} 1, \mathrm{P} 2$, etc. postnatal day 1 , postnatal day 2 , etc.

PBS phosphate buffered saline

PCR polymerase chain reaction

PFA paraformaldehyde

$\mathrm{PH}$ domain pleckstrin homology domain

SNAP soluble NSF attachment protein

SNAP25 synaptosomal-associated protein 25

SNARE SNAP receptor

STED stimulated emission depletion

SV Synaptic vesicle

TRIS tris(hydroxymethyl)aminomethane

VAMP2 vesicle-associated membrane protein 2

VAMP4 vesicle-associated membrane protein 4

VAMP7 vesicle-associated membrane protein 7

vATPase vesicular ATPase

VGAT vesicular GABA transporter

VGlut vesicular glutamate transporter

Vti1a vesicle transport through interaction with t-SNAREs homologue 1a 


\section{List of Publications}

Truckenbrodt $\mathbf{S}^{\dagger}$, Rizzoli SO (2015) Synaptic Vesicle Pools: Classical and Emerging Roles. In: Presynaptic Terminals (Springer Japan, Editor: Sumiko Mochida), pp 329-359, doi: 10.1007/978-4-431-55166-9_14

Wilhelm BG, Mandad S*, Truckenbrodt S*, Kröhnert K, Schäfer C, Rammner B, Koo SJ, Claßen GA, Krauss M, Haucke V, Urlaub H, Rizzoli SO (2014) Composition of Isolated Synaptic Boutons Reveals the Amounts of Vesicle Trafficking Proteins. Science 344:10231028, doi: 10.1126/science.1252884

Revelo $\mathrm{NH}^{\star}$, Kamin $\mathrm{D}^{*}$, Truckenbrodt S, Wong AB, Reuter K, Reisinger E, Moser T, Rizzoli SO (2014) A new probe for super-resolution imaging of membranes elucidates trafficking pathways. J Cell Biol 205(4):591-606, doi: 10.1083/jcb.201402066

Truckenbrodt $\mathbf{S}^{\dagger}$, Rizzoli SO ${ }^{\dagger}$ (2014) Spontaneous Vesicle Recycling in the Synaptic Bouton. Front Cell Neurosci 8:409, doi: 10.3389/fncel.2014.00409

*equal contribution

${ }^{\dagger}$ corresponding author 


\section{Curriculum Vitae}

\section{Education}

09/2011 University of Göttingen | International Max Planck Research School for

present Molecular Biology (fast-track PhD programme), grade of first year exam: 1.25

09/2011 Distance University in Hagen | MA in Studies of Modern Europe - History and present Literature (current average grade: 1.15 )

$07 / 2011$

$06 / 2007$

University of Würzburg | BSc in Biomedicine, grade: 1.5

Gymnasium Ernestinum Coburg | high school diploma (Abitur), grade: 1.2

\section{Research Experience}

10/2012 University of Göttingen Medical Centre | PhD student investigating the

present molecular architecture of the synapse and long-term dynamics of synaptic vesicle pools with Prof. Silvio O. Rizzoli.

05/2012 MPI for Biophysical Chemistry, Göttingen | Lab rotation student investigating

06/2012 SNARE palmitoylation and membrane partitioning with Prof. Reinhard Jahn \& Dr. Geert van den Bogaart.

02/2012 European Neuroscience Institute, Göttingen | Lab rotation student

$04 / 2012$ investigating the life cycle of synaptic vesicles, their recycling rate, and functional transitions with Dr. Silvio O. Rizzoli.

01/2012 MPI for Experimental Medicine, Göttingen | Lab rotation student working on

02/2012 application and design of FRET-based metabolic sensors with Dr. Moritz Rossner.

05/2011 University of Würzburg | Bachelor's Thesis: Evaluation of Novel Optogenetic

07/2011 Tools - GCaMP3 and Cameleon 2.1 in in Vivo Calcium Imaging (grade: 1.0) with Prof. Erich Buchner.

03/2010 University of Würzburg | Student research assistant establishing a novel

08/2010 approach to study the neural network of Drosophila melanogaster by direct manipulation, using Channelrhodopsin, in a behavioural setup with Prof. Erich Buchner.

\section{Awards and Achievements}

- 12/2015: honorary mention in the Elevator Speech Contest of the American Society for Cell Biology Annual Meeting 2015

- 09/2014: Society for Neuroscience (USA) Annual Meeting Travel Award

- 02/2013 - 02/2016: Excellence Stipend of the Göttingen Graduate School for Neurosciences, Biophysics, and Molecular Biosciences (GGNB)

- $10 / 2010: 1^{\text {st }}$ price in the Writing Contest at the $5^{\text {th }}$ International PhD Student Symposium of the Graduate School for Life Sciences in Würzburg, Germany 


\section{Extracurricular Activities \& Public Outreach}

- Editor in Chief for the $1^{\text {st }}$ Edition of the Newsletter "GGNB Times" for the Göttingen Graduate School for Neurosciences, Biophysics, and Molecular Biology (GGNB)

- Journey to your Senses at "Nacht des Wissens" (Night of Knowledge), Göttingen, Germany - hands-on science booth of the lab at a city-wide science fair $(\sim 20,000$ attendants), $17^{\text {th }}$ February 2015

- "denkBAR" Göttingen - public forum on the history of synaptic neuroscience and own current resarch, $\sim 100$ attendants, $11^{\text {th }}$ March 2014

- presentation of lab and own current research to the social club "Bärengemeinde Göttingen", $\sim 20$ attendants, $28^{\text {th }}$ June 2013

- 05/2013-05/2015: PhD student representative of the IMPRS for Molecular Biology

- 10/2012 - 10/2014: one of five main organisers of the 10th and 11th international PhD student symposium "Horizons in Molecular Biology" (appr. 20 international speakers \& 300 participants), Göttingen; responsible for Public Relations \& Advertisement, Design, Finance, and Co-ordination

\section{Teaching Experience}

since Supervisor | MD Thesis: Evaluation of Novel Expansion Microscopy Techniques 03/2016 for Imaging Brain Slice Preparations and Cultured Cells

05/2015 Supervisor | graduate student lab rotation (2 months): Two Novel Constructs for 06/2015 Staging and Silencing Synaptic Vesicles - sTage and SYLENT

03/2014 Tutor | graduate level practical course: Super-resolution Microscopy and Physiology of Synapses

08/2013 Supervisor | MSc Thesis (7 months): Examination of the Molecular Composition 02/2014 of the Recycling versus the Reserve Pool of Synaptic Vesicles.

02/2013 Supervisor | 3 independent undergraduate research internship ( $\sim 4$ weeks each): 08/2013 Synaptic Vesicle Physiology; one guest student from Vienna, Austria, and two students from Göttingen, Germany

01/2013 Tutor | various graduate level theoretical courses: Architecture of the Cell (1x), present Biological Membranes II (2x), Synaptic Transmission \& Integration (2x), C. elegans as a Model Organism (1x)

07/2011 Tutor | undergraduate level practical course: Drosophila as a model organism.

\section{Journal Publications}

Wilhelm BG, Mandad S*, Truckenbrodt S*, Kröhnert K, Schäfer C, Rammner B, Koo SJ, Claßen GA, Krauss M, Haucke V, Urlaub H, Rizzoli SO (2014) Composition of Isolated Synaptic Boutons Reveals the Amounts of Vesicle Trafficking Proteins. Science 344:10231028. *equal contribution

Revelo $\mathrm{NH}^{*}$, Kamin $\mathrm{D}^{*}$, Truckenbrodt S, Wong AB, Reuter K, Reisinger $\mathrm{E}$, Moser T, Rizzoli SO (2014) A new probe for super-resolution imaging of membranes elucidates trafficking pathways. J Cell Biol 205(4):591-606. *equal contribution 


\section{Reviews \& Book Chapters}

Truckenbrodt $\mathrm{S}^{\dagger}$, Rizzoli SO (2015) Synaptic Vesicle Pools: Classical and Emerging Roles. In: Presynaptic Terminals (Springer Japan, editor: Sumiko Mochida) pp 329-359. ${ }^{\dagger}$ corresponding author

Truckenbrodt S ${ }^{\dagger}$, Rizzoli SO ${ }^{\dagger}$ (2014) Spontaneous Vesicle Recycling in Synaptic Boutons. Front Cell Neurosci 8:409. ${ }^{\dagger}$ corresponding authors

\section{Conference Contributions - Talks}

A Molecular Timer Inactivates Ageing Synaptic Vesicles. Selected Talk. American Society for Cell Biology Annual Meeting. San Diego, United States of America, 12th-16th December 2015

The Mechanisms of Synaptic Vesicle Ageing. Invited Talk. The 38th Annual Meeting of the Japan Neuroscience Society. Kobe, Japan, 28th-31st July 2015.

A Molecular Timer Inactivates Ageing Synaptic Vesicles. Selected Talk. Neurizons Conference - Notes of an Orchestrated Brain. MPI for Biophysical Chemistry, Göttingen, Germany, 26th-29th May 2015.

The Synapse Nanomap. Invited Talk. Joint Berlin-Göttingen Neuroscience Retreat, organised by IMPRS for Neurosciences, Göttingen \& MDC Research School Molecular Neurobiology, Berlin. Lychen, Germany, 7th-9th September 2014.

The Molecular Organisation of the Synaptic Bouton. Selected Talk. 4th LIN-Symposium on Synaptic Function and Synaptic Pathology of Disease, organised by the Leibniz Intsitute for Neurobiology. Tangermünde, Germany, 25th-28th August 2014.

Effects of Selective Neuronal Activation of the Protocerebral Bridge on the Walking Behaviour of Drosophila melanogaster. Contributed Talk. 4th Day of Molecular Medicine. University of Ulm, Germany, 16th April 2011.

\section{Workshops \& Seminars}

Truckenbrodt S. Acquiring Key Competences by Organising Scientific Meetings as a PhD Student - Horizons in Molecular Biology. Workshop. Annual Meeting of the German Society for Key Competences in Teaching, Research and Practice. Göttingen, Germany, $1^{\text {st }}-3^{\text {rd }}$ September 2014.

Truckenbrodt S. Data Visualisation in the Life Sciences - The First 3D Model of a Synapse. Seminar. Summer School Visual Analysis, Göttingen Centre for Digital Humanities. Göttingen, Germany, $28^{\text {th }}$ July to $1^{\text {st }}$ August 2014.

Truckenbrodt S. Synaptic Vesicles Work, Age, and Retire in a Complex 3D Environment The Structural and Functional Organisation of the Presynaptic Terminal. Seminar for guest students from Spain. XLAB (teaching lab for young scientists), Göttingen, Germany, $23^{\text {rd }}$ July 2015 


\section{Conference Contributions - Posters}

Truckenbrodt S, Viplav A, Jähne S, Vogts A, Richter K, Denker A, Fornasiero EF, Rizzoli SO. A molecular timer tracks synaptic vesicle use to prevent the participation of aged vesicles in synaptic transmission. Janelia Farm Conference on Molecular Mechanisms in the Synapse: Experiments and Modeling. Ashburn, United States of America, 02nd-05th May 2016

Truckenbrodt S, Viplav A, Jähne S, Vogts A, Richter K, Denker A, Fornasiero EF, Rizzoli SO. A Molecular Timer Inactivates Ageing Synaptic Vesicles. American Society for Cell Biology Annual Meeting. San Diego, United States of America, 12th-16th December 2015

Truckenbrodt S, Viplav A, Jähne S, Vogts A, Richter K, Denker A, Fornasiero EF, Rizzoli SO. A Usage-dependent Mechanism Measures Synaptic Vesicle Age and Prevents the Use of Old Vesicles in Synaptic Transmission. Society for Neuroscience Annual Meeting. Chicago, United States of America, 17th-21th October 2015.

Truckenbrodt S, Viplav A, Richter K, Denker A, Fornasiero EF, Rizzoli SO. The Age of the Synaptic Vesicle Determines Its Ability to Release. 12th Horizons in Molecular Biology. MPI for Biophysical Chemistry, Göttingen, Germany, 14th-17th September 2015.

Truckenbrodt S, Viplav A, Richter K, Denker A, Fornasiero EF, Rizzoli SO. A Molecular Timer Inactivates Ageing Synaptic Vesicles. European Synapse Meeting. Bristol, United Kingdom, 7th-9th September 2015.

Truckenbrodt S, Wilhelm BG, Mandad S, Kröhnert K, Schäfer C, Rammner B, Koo SJ, Claßen GA, Krauss M, Haucke V, Urlaub H, Rizzoli SO. The Molecular Organisation of the Synaptic Bouton and its Physiological Implications. European Biophysics Congress. Dresden, Germany, 18th-22nd July 2015

Truckenbrodt S, Viplav A, Denker A, Fornasiero EF, Rizzoli SO. A Molecular Timer Inactivates Ageing Synaptic Vesicles. The Age and Usage of the Synaptic Vesicle Determine its Ability to Release. Neurizons Conference - Notes of an Orchestrated Brain. MPI for Biophysical Chemistry, Göttingen, Germany, 26th-29th May 2015.

Truckenbrodt S, Viplav A, Denker A, Fornasiero EF, Rizzoli SO. The Age of the Synaptic Vesicle Determines its Ability to Release. Assembly and Disassembly of the Nervous System. Weizmann Institute of Science, Rehovot, Israel, 19th-21st January 2015.

Truckenbrodt S, Wilhelm BG, Mandad S, Kröhnert K, Schäfer C, Rammner B, Koo SJ, Claßen GA, Krauss M, Haucke V, Urlaub H, Rizzoli SO. The Molecular Organisation of the Synaptic Bouton. Society for Neuroscience (USA) Annual Meeting. Washington, D.C., United States of America, 15th-19th November 2014.

Truckenbrodt S, Wilhelm BG, Mandad S, Kröhnert K, Schäfer C, Rammner B, Koo SJ, Claßen GA, Krauss M, Haucke V, Urlaub H, Rizzoli SO. The Molecular Organization of Membrane Trafficking at the Synaptic Bouton. SFB 638 International Symposium on Macromolecular Complexes in Biosynthetic Transport. Heidelberg, Germany, 6th-7th October 2014.

Truckenbrodt S, Wilhelm BG, Mandad S, Kröhnert K, Schäfer C, Rammner B, Koo SJ, Claßen GA, Krauss M, Haucke V, Urlaub H, Rizzoli SO. The Molecular Organisation of the Synaptic Bouton. 4th LIN-Symposium on Synaptic Function and Synaptic Pathology of Disease, organised by the Leibniz Intsitute for Neurobiology. Tangermünde, Germany, 25th-28th August 2014. 
Truckenbrodt S, Viplav A, Rizzoli SO. Long-term Temporal Dynamics of Functionally Distinct Pools of Synaptic Vesicles. GGNB Science Day. MPI for Biophysical Chemistry, Göttingen, Germany, 9th December 2013.

Truckenbrodt S, Wilhelm B, Rizzoli SO. The Nanoscale Map of a Synaptic Bouton. 4th Argentina-Germany Bilateral Workshop. University of Göttingen, Germany, 13th-15th June 2013.

Truckenbrodt S, Denker A, Rizzoli SO. Investigating the Behaviour of Synaptic Vesicles with Immunostaining Methods. 9th Horizons in Molecular Biology. MPI for Biophysical Chemistry. Göttingen, Germany, 8th-11th October 2012. 